\title{
LEGIBLITY
}

A major purpose of the Techni-

cal Information Center is to provide the broadest dissemination possible of information contained in DOE's Research and Development Reports to business, industry, the academic community, and federal, state and local governments.

Although a small portion of this report is not reproducible, it is being made available to expedite the availability of information on the research discussed herein. 


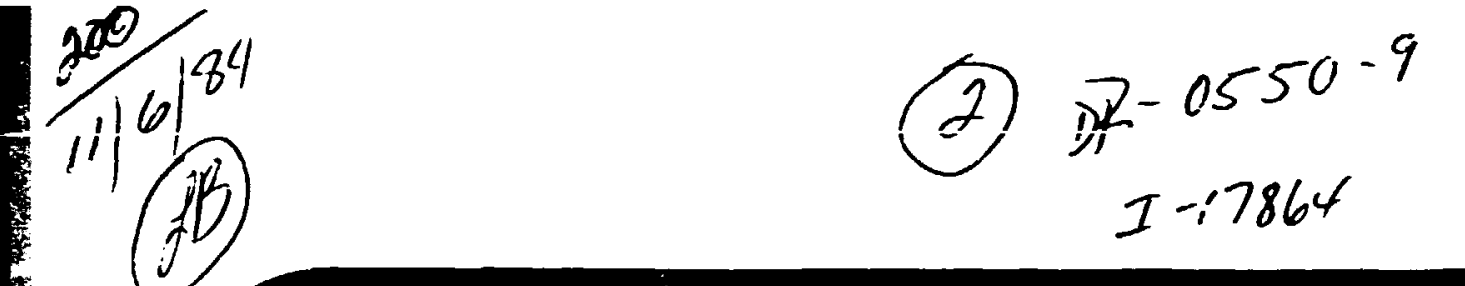

\section{TOKAMAK FUSION CORE EXPERIMENT: DESIGN STUDIES BASED ON SUPERCOADUCTING AND HYBRID TOROIDAL FIELD COILS}

Design Overview

HOTIGE

PARTIOKS OF THIS REPORT ARE ILLEGI:HE. It has been reproduced from the best ayailabic copy to permit the broadest u.ass!cie availability.

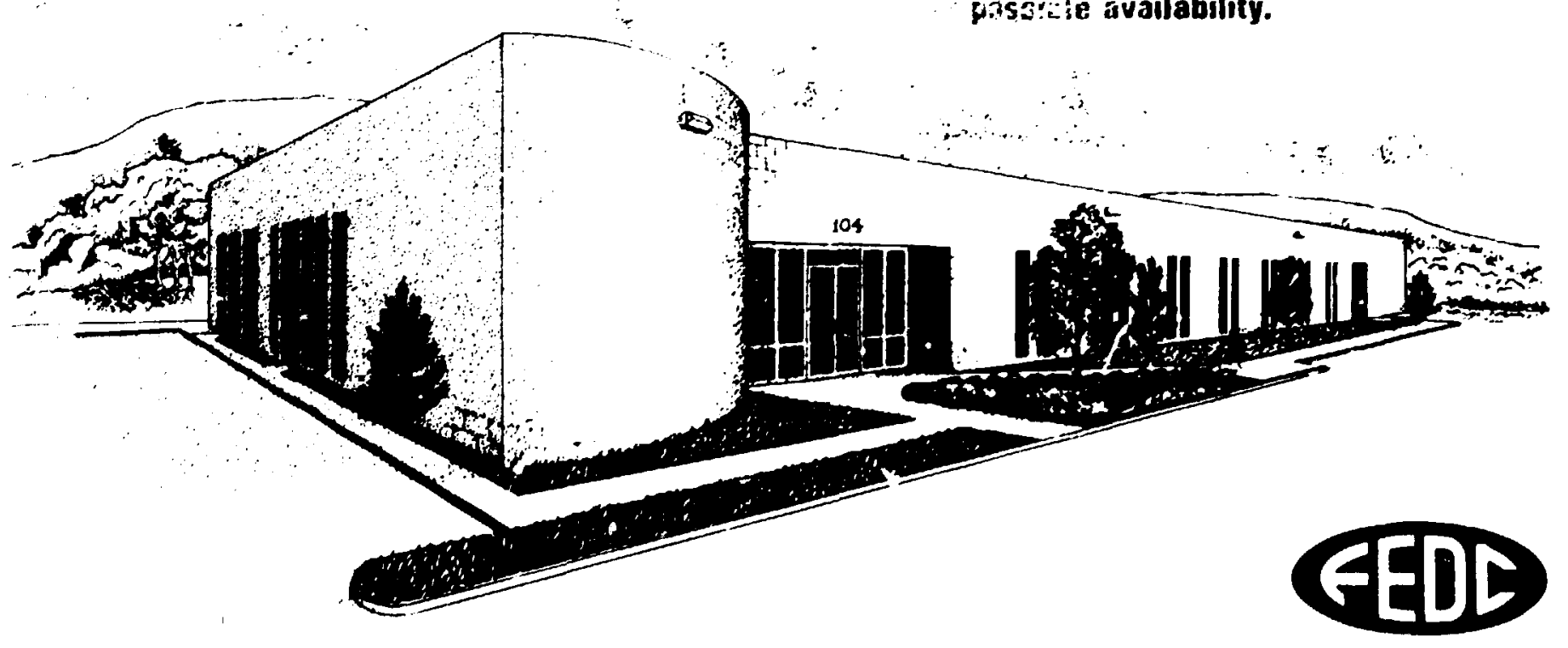

FUSION ENGINEERING DESIGN CENTER

Oak Ridge National Laturatory * Oak Ridge, Tennessee 
OKNL/FEUC-84/3

Dist. Categor: UC- $20 \mathrm{c,d}$

On:iis/FEDC- $-84 / 3$

D.05 090428

TOKAMAK FUSION CORE EXPERIMENT:

DESIGN STUDIES BASED ON SUPERCONDUCTING

AND HYBRID TOROIDAL FIELD COILS

DESIGN OVERVIEW

Edited by

C. A. Flanagan

Date Published - October 1984

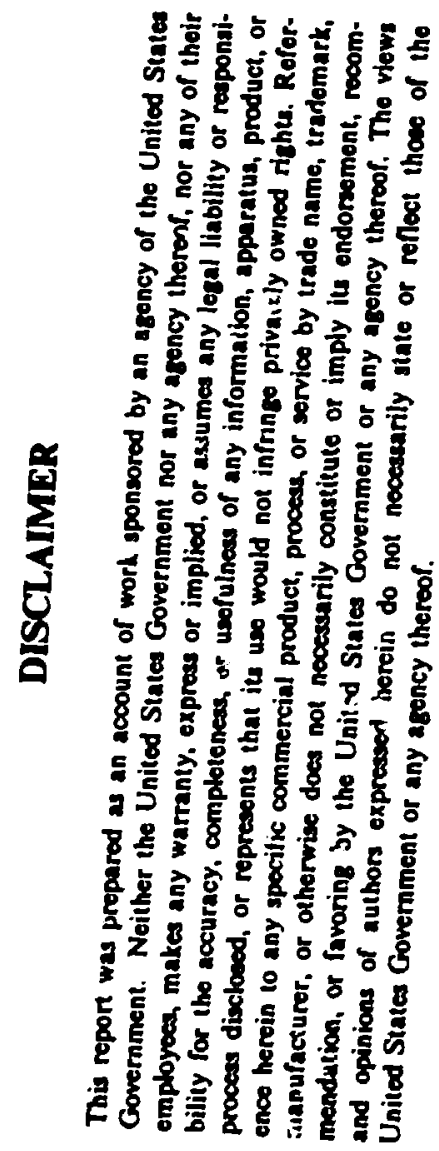

Prenared by the

OAK RIDGE NATIONAL LABORATORY

Oak Ridge, Tennessee 37830

operated by

MARTIN MAR IETTA ENERGY SYSTEMS, INCORYORATEI

for the

DEPARTMENT OF ENERGY

und 2 r cont ract DE-ACOS-840R 21400

MASTER 
CONTENTS

LIST OF FIGURES ...................... vii LIST OF TARLES ......................... . . . . . ix CONTRIBUTORS TO TFCX DESIGN STUDIES . . . . . . . . . . . . xi ACKNOWLEDGMENTS . . . . . . . . . . . . . . . xiii ABSTRACT . . . . . . . . . . . . . . . $x V$

1. INTRODUCTION ................... 1-1

2. ISSUES AND OFTIONS .................. 2-

3. PHYSICS CONSIDERATIONS ............... 3-

4. CONFIGURATION FEATURES ................ . . . . 4-1

5. PlASMA OPERATING SCENARIO .............. 5-1

5.1 Prefill and Ionization ........... . . 5-j

5.2 Current Initiation and Ramp-Up ......... 5-j

5.3 Bulk Heating ............. 5-3

5.4 Burn ................ 5-4

5.5 Shutdown ................. 5-5

5.6 Pumpdown ............... . . . 5-5

6. SYSTEMS STUDIES ................. 6-1

6.1 Driven-Current Tokamak (DCT) Scoping Studies .... 6-1

6.2 DCT-8 Configuration Trade Study . . . . . . . 6-3

6.3 Systems Results For TFCX-S and TFCX-H . . . . . 6-6

7. MAGNETIC SYSTEMS ................. . . 7 - 1

7.1 Superconducting TF coils ........... 7-1

7.2 pF coil System .............. $7-11$

7.3 Cryostat ................... $7-12$

8. NUCLEAR SYSTEMS ................ 8-1

8.1 Torus and Shield Design ............. 8-1

8.2 first Wall System . . . . . . . . . . . 8-5

8.3 Impurity Control System . . . . . . . . . . 8-7

9. Plasm HeATLNG SYSTEMS . . . . . . . . . . . . . . 9-

9.1 Plasma Initiation and Startup........... . 9-1

9.2 Heating and Current Drive............ . . 9-3 
10. ElECTRICAL SYSTEMS ................. 10-1

10.1 Power Handling and Conversion ........... 10-1

10.2 Energy Storage . . . . . . . . . . . 10-3

10.3 Plasma Diagnostics, Information, and Control Systems - 10-3

11. AUX ILIARY SYSTEME ................. . . 11-1

11.1 Fueling System ................. 11-1

11.2 Vacurm Pumping ............... . . 11-2

11.3 Tritium Systems . ............... . 11-10

11.4 Remote Maintenance Equipment ........... 11-11

12. FACILITIES . . . . . . . . . . . . . 12-1

13. CONCLUDING REMARKS ..................... 13-1 


\section{FOREWORD}

This overview document summarizes the scoping studies and preconceptual design studies performed for the Tokamak Fusion Core Experimeut (TFCX) class of device during FI 1983. The studies reported here are for candidate TFCX design options that use superconducting toroidal field (TF) coils. The FY 1984 studies will continue the present effort and will be expanded to include TFCX design options that use resistive copper TF coils. 


\section{LIST OF FIGURES}

Figure

$\underline{\text { Page }}$

4.1 TFCX-S elevation view ................ 4-4

4.2 TFCX-S plan view. ................... . $t-5$

1.j Elevation and plan view schematics of TFCX-H. . . . ... 4-6

6.: Flow diagram of system study procedure. . . . . . . . 6-2

6.2 DCT configuration oprions ............... 6-5

7.1 Overall arrangement of the magnets. ........... 7-2

7.2 Arrangement of $\mathrm{OH}$ solenoid and superconducting EF ring coils in the TFCX-S design. . . . . . . . . . . . . 7-13

8.1 TFCX-S general configuration. . . . . . . . . . 8-2

8.2 TrCX-H general arrangement. . . . . . . . . . . 8-3

8.3 TFCX first wall design concepts . . . . . . . . . . 8-6

8.4 Plan view of installed limiter segments . . . . . . . 8-8

8.5 TFCX limiter location . . . . . . . . . . . . . 8-9

9.1 TFCX ECRH system. . . . . . . . . . . . . . . . 9-7

9.2 LHRH launcher interface with torus. . . . . . . . . . 9-8

9.3 ICRH launcher interface . . . . . . . . . . . . . . 9-9

11.1 Vacuum pumping system . . . . . . . . . . . . . . 11-7

11.2 Tritium flow paths for TFCX tritium processing units. . . 11-12

12.1 TFCX Fusion Island. . . . . . . . . . . . . . . 12-2

12.2 TFCX plot plan. . . . . . . . . . . . . . . 12 3 


\section{LIST OF TAF,LES}

Table

Page

4.1 Key systems that dominate tokamak configuration designs. . . 4-1

4.2 Key parameters for TFCX-S and TFCX-H . . . . . . . 4-7

5.1 TFCX plasma operating scenario (D-T operation) ...... 5-2

6.1 Key parameters for DCT- 2 and DCT-8 . . . . . . . 6-4

6.2 TFCX design guidelines and criteria. . . . . . . . . 6-7

$6.3 \mathrm{Key}$ parameters for TFCX design options......... 6- .

7.1 FED magnetic system parameters (TFCX-S). . . . . . . . 7-3

7.2 TF coil current densities. . . . . . . . ... 7-6

$7 .:$ TFCX TF magnet system parameters . . . . . . . . . . 7-10

9.1 ECRH preheating requirements for TFCX-S and TFCX-H . . . 9-2

9.2 Current-drive requirements for TFCX-S and TFCX-H . . . 9-4

9.3 Bulk heating requirements. . . . . . . . . . . . 9-5

10.1 Major Ac power system loads. . . . . . . . . . . . 10-2

10.2 Essential power requirements . . . . . . . . . . . . 10-4

11.1 Pellet injector requirements for TFCX. . . . . . . . .11-3

11.2 Gas injector requirements. . . . . . . . . . . . . .11-4

11.3 Guidelines for TFCX vacuum pumping system performance • . .11-6

11.4 Vacuum pump paraneters . . . . . . . . . . . . .11-9

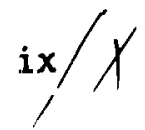


FEDC
R. J. Barrett
S. K. Borowski
T. G. Brown
M. K. Bullock
G. T. Bussell
C. A. Flanagan
G. M. Ful Ier
J. j. Galambos
G. E. Gorker
J. R. Haines
L. M. Hively
R. J. Hooper
S. S. Kalsi
M. H. Kunselman
V. D. Lee

$\underline{\text { ANL }}$
C. C. Baker
P. A. Finn
Y. Gohar
R. F. Mattas

S. K. Makres

D. H. Metzler

J. B. Miller

J. G. Murray

C. E. Nelson Y-K. M. Peng

R. L. Reid

W. T. Reiersen

K. E. Rothe

T. E. Shannon

P. T. Spampinato

V. C. Srivastava

D. J. Strickler

F. W. Wiffen

LANL

J. D. Rogers

D. B. Montgomery

R. J. Thome

J. Schultz 


\section{ACKNOWLEDFMENTS}

The Tokanak Fusion Core Experiment (TFCX) design studies reported here have been performed in FY 1983 by the Fusion Engineering Design Center (FEDC) in collaboration with the Princeton Plasma Physics Laboratory (PPPL), with broad support from the fusion community. Particular thanks go to J. A. Schmidt and G. V. Sheffield of PPPL, who provided stimulating guidance and leadership for much of this effort; to P. Stone and A. Opdenaker of the Office of Fusion Energy, who provided direction of this reactor studies program; and to T. E. Shannon, who provided FEDC leadership. Finally, special thanks go to M. Bullock, P. J. Fogarty, M. H. Kunselman, and S. K. Makres for expert support in preparation of many of the drawings and figures and to Linda Caldwell, Melinda Cofer, Shirlene Dale, Nancy Iroy, and Patty Powell for outstanding professional secretarial support throughout the year.

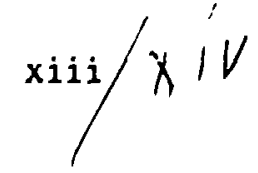


This document is a design overview that describes the scoping studies and preconceptual design effort performed in FY 1983 on the Tokamak Fusion Core Experiment (TFCX) class of device. These studies focussed on devices with all-superconducting toroidal field (TF) coils and on devices with superconducting TF coils supplemented with copper TF coil inserts located in the bore of the TF coils in the shield region. Each class of device is designed to satisfy the mission of ignition and long pulse equilibrium burn. Typical design parameters are: major radius $=3.75 \mathrm{~m}$, minor radius $=1.0 \mathrm{~m}$, field on axis $=4.5 \mathrm{~T}$, plasma current $=7.0 \mathrm{MA}$. These designs rely on lower hybrid (LHRH) current rampup and heating to ignition using ion cyclotron range of frequency (ICRF). A pumped limiter has been assumed for impurity control. The present document is a design overview; a more detailed design description is contained in a companion document .
\end{abstract}




\section{INTRODUCTION}

In the fall of 1982, the Magnetic Fusion Advisory Committee (MFAC) considered numerous upgrades of the Tokamak Fusion Test Reactor (TFTR), including new devices. The Princeton Plasma Physics Laboratory (PPPL) recommended (and the MFAC endorsed) an in-depth study of a superconducting long-pulse ignition device. Accordingly, during FY 1983, engineering studies have been pursued exploring this class of device. The preconceptual studies are being carried out at the Fusion Engineering Design Center (FEDC) and PPPL, with broad community support: to establish the characteristics of the :lext large tokamak experimental device.

The mission for this device, designated the Tokamak Fusion Core Experiment (TFCX), is to achieve ignition and long-pulse equilibrium burn. To the extent practical, it is desirable to have the device serve as a focus for the development of future fusion technologies. It is important to the project that the cost of the device be minimized. Accordingly, high-fluence nuclear testing has not been incorporated into the mission, and the nuclear testing objectives will be relegated to other future fusion programs to achieve.

Devices with the following toroidal field (TF) coil options are leing considered for the TFCX facility: (1) an all-superconducting option (TFCX-S), (2) a copper-superconducting hybrid option (TFCX-H), and (3) an all-copper option (TFCX-C). It is planned that a shoice be made foilowing a preconceptual design for these candidates. These preconceptual design studies will be carried to a level that not only ensures design feasibility within the subsystem envelopes daveloped but also provides a basis for estimating the associated subsystem costs.

The efforts in FY 1983 focussed predominantly on the all-superconducting option and on the copper-superconducting hybrid option. For these two options, design studies were completed to establish the overall characteristics of each class of device to establish the design definition of all of the major subsystems, and to develop associated capital cost projections. This paper describes the TFCX studies performed in FY 1983. The studies will continue in FY 1984 with the focus on developing allcopper TF coil options. It is also intended to make a selection of 


$$
1-2
$$

options in mid-FY 1984 and to initiate a conceptual design using the results to date as a point of departure.

It should be noted that no decision has been made un a final design for any of the three options and considerable flexibility exists in establishing the final size and associated performance parameters for each option. Included in establishing the final parameters and size will be evaluations and decisions on the physics basis to be used. This will be done in FY 1984. However, for purpose of discussion of key features of eash option, nominal cases are presented in this paper for the options studied to date.

Section 2 discusses issues and options of interest to TFCX. Secticn 2 discusses physics considerations. Section 4 presents a decription of configuration features. Section 5 discusses the plasma operating scenario consideration. Section 6 summarizes numerous systems studies performed using the tokamak systems code. Sections 7 through 11 describe, respectively, the magnetic, nuclear, plasma heating, electrical, and auxiliary systems. Section 12 discusses the facilities considerations.

Background intomatinn leading to these TFCX design studies can be found in $R=f$. 1. A more detailed design description, together with the supporting trade studies, can be found in Ref. 2 . 


\section{$\therefore \quad$ ISSUES AND OPTIONS}

The TFCX (along with complementary, nonfusion facilities) must address the remaining issues requiring resolution before a final commitment is made to all Engineering Test Reactor (ETR). The physics and technology issues to be addressed by TFCX will be those remaining after the operation of the Doublet III Upgrade (DIII-U), Fusion Test Reactor (TFTR), and the Joint European Torus (JET).

Most of the issues addressed by TFCX will be related to ignition and/or long-pulse operation. Information will be required on postignition confinement and burn control, on maintaining a high-beta configurdcion for a period which is long compared with magnetic diffusion times, on impurity control and power/particle handling at long pulse and with a reactor-like core plasma, on initiating plasma currents using noninductive current drive, and on ash accumulation and removal. Closely related technology issues are testing of ion cyclotron and lower hybrid launcher concepts designed for the radiation environment, for tritium containment, and for maintainability and management of disruptive energy dumps at more nearly reactor-relevant levels (5 to $10 \mathrm{MA}$ and $\geqslant 100 \mathrm{MJ}$ ).

The next tokamak facility, along with all advanced fusion devices, must provide information on component and system reliability and availability. Reactor-relevant TF coils must be employed on cperating tokamaks; however, no existing or planned U.S. tokamak uses superconducting coils. Enlightenment on maintenance is likely to come exclusively from operating devices. Before a next tokamak, only TFTR must face up to remote maintenance, and unfortunately, the TFTR configuration is unsuitable for reactor use. Thus, remote maintenance as well as component and system reliability will be important issues addressed by TFCX. Other systems-related issues are tritium management and the development of diagnostics, data acquisition, and control systems for the full fusion environment, including ignition and long-pulse burn.

Most of the issues to be resolved by the next tokamak facility are associated with ignition and long-pulse burn. Therefore, there are three preferred options for the next tokamak facility that can provide 
ignition and long-pulse burn capability. These three options are distinguisned by the type of TF magnet employed: all-superconductor, a copper-superconducting combination, or all-copper.

The TFCX option with a superconducting TF coil is clearly relevant to future reactor development. However, neutron shielding is required to reduce the nuclear heating in the superconducting coil even for lowfluence operation, and this takes up space and tends to increase the major radius. In addition, the maximum reljable operating field realizable with superconducting coils is substantially less than tinat for copper coils. The lower field results in an increase in device size for a given ignition parameter. The increased plasma major radius and the aspect ratio associated with superconducting coils are in the direction to incur a potential cost penalty relative to the copper coil option.

Using copper-superconductor combiration TF coils provides a potentially attractive hybrid option. Copper TF coils located inside the superconducting TF coils provide shielding for the superconducting coils and higher central Fields, resulting in smaller size and potentially lower cost than the all-superconductor option. This options's advantage over the all-superconductor approach is the higher central field at the same or lower cost. The advantage over the all-copper option is its increased relevance to the ETR issues: it does develop superconducting coils and its design is based on these coils. Disadvantages relative tc the all-copper options are a somewhat longer schedule and increased complexity.

Using all-copper TF coils removes the superconducting design requirement for shielding the TF coils, potentially reducing size and complexity, and allows higher central fields, allowing an additional decrease in size for equivalent performance. In addition, the need for superconducting coil research and development is eliminated. The principal advantages of the all-copper device are the technical simplicity, reliability, and a shorter construction schedule. The principal dis.advantage arises directly from the advantages: the copper coils, the attendant simplicity, and the lack of an accompanying superconducting coil development causes this option to be least relevant to the unresolved superconducting issues of an ETR. 


\section{PHYSICS CONSIDERATIONS}

The overall mission for TFCX dictates that the physics requirements For the device te compatible with a long-pulse, ignited discharge. The key considerations in assessing the prospects for ignition are the quality of confinement and the limits en plasma pressure (beta limits). During initial studies, conventional plasma shapes (D shapes) and associated modest betas have been assumed.

Experiments on present devices indicate a soft beta limit, which may provide a stable operating point during burn. However, some method of varying the operating point (such as varying plasma current, ripple, or impurities) may be required.

The early Fusion Engineering Device (FED) and International Tokamak Reactor (INTOR) design studies were carried out using Alcator scaling for energy confinement and using beta 1 imits roughly consistent with ideal magnetohydrodynamic (MHD) theory. A useful approximate form for the beta limit, deduced from a myriad of low-q MHD stability code runs and associated heating experiments, is

$$
\langle\beta\rangle_{\max }=0.2 \frac{\mathrm{a}}{\mathrm{Rq}} \frac{1+k^{2}}{2} \text {, }
$$

where $\langle\beta\rangle$ is the average beta, $a$ is the plasma minor radius, $k$ is the plasma elongation, $R$ is the major radius, and $q$ is the safety factor. The observation in Doublet III in 1983 of a stable, elongated discharge with a beta of $4.5 \%$ is consistent with this formulation.

With regard to confinement, the results of a number of auxiliary heating experiments are in conflict with Alcator scaling. A better fit to such data is

$$
\tau_{E} \propto I_{p}(M A) \text { a (m) } f(k),
$$

where $\tau_{E}$ is the energy confinement time, $I_{p}$ is the plasma current, $a$ is the plasma minor radius, and $f(K)$ is a modifying coefficient that depends on plasma geometry. The size scaling is subject to considerable uncertainty. 
The ignition parameter $I$ is defined as the ratio of the alpha heating power $P_{\alpha}$ to the plasma loss power $P_{\text {Loss }}$. Since $P_{\alpha} \propto B^{2} \mathrm{E}^{4}$ and $P_{\text {Loss }} \propto B B^{2} / \tau_{E}$, one finds using the reiationship

$$
q \propto \frac{B_{T}}{I_{p}} \quad \frac{2}{R} a f(k)
$$

that the above equations can be combined to obtain a simple form for the ignition parameter:

$$
I=\frac{P_{\alpha}}{P_{1 \text { loss }}} \propto I_{p}^{2} B_{T} .
$$

When one is this close to the beta limit, there is an observed dependence of confinement on beta which must be taken into account when estimating the ignition parameter.

A single-null poloidal divertor configuration was developed as part of the INTOR desigr. studies with minimum impact on the polidal field (PF) coil design and mechanical configuration. This configuration can be accommodated within about the same envelope required for a pumped limiter placed at the bottom of the plasma chamber. Due to the reasonable compatibility of these two configurations, the TFCX preconcept al design studies and the associated option selection have been designed with a pumped limiter. Ongoing divertor and limiter experiments will form the basis for selecting the impurity control option during the conceptual design.

Current ramp-up at low density with $r$ heating near the lower hybrid frequency [loser hybrid resonanic heating (LHRH)] has been successful in several recent tokamak experiments. This technique will be used with a modest ohmic heating $(\mathrm{OH})$ voltage to ramp the plasma current during startup. This will alleviate the inductive volt-second requirement for startup and conserve the volt-seconds for use during the burn. The studies performed in FY $198 j$ assume a 300-s ignited burn. About $10 \mathrm{MW}$ of LHRH appears sufficient for current ramp-up purposes. The remaining $20 \mathrm{MW}$ required for heating to ignition is provided with ion cyclotron range of frequency (ICRF) heating. A modest amount $(0.1$ to $1 \mathrm{MW}$ ) of 
electron cyclotron resonance heating (ECRH) may be required for preionization and has been incorporated.

Some aspects of the physics basis for the TFCX will require develop...nt and qualification on existing experiments. High-power heating experiments on TFTR, JET, DIII-D, and the Poloidal Beta Experiment (PBX) will confirm the chuice of the transport crefficients and beta limits used for the TFCX design. Initial high-power neating experiments on TFTR and JET are well timed to fit the TFCX needs. These experiments wili provide confinement data as wel: as the physics information necessary for the choice of the impurity control optiun for the TFCX. If TFTR and JET operate successfully without divertors, then impurity contro: ith the pumped limiter on the TFCX will be the logical choice. I $\hat{r}$ a divertor proves necessary, then additional informatica on divertor design will be needed and will depend mainly or experiments $0_{1}$ Axisymnetric Divertor Experiment-Upgrade (ASDEX-U) at Garching.

The present TFCX design options rely heavily on lower hybrid current drive (LHCD) current ramp-up to reduce the volt-second requirci...nts and associated central solenoid size. This technique shows great promise; howev $\epsilon$, further development of the LHCD data base will be required. Lower hybrid current ramp-up is being studied on the Princeton Large Torus (PLT) and Alcator $C$.

The TFCX project will require qualification of ICR! for heating to ignition. Programs are in place to provide the development of $\mathrm{rf}$ launcheis and the qualification of ICRF necessary to proceed with the TFCX and will test a range of candidate launcher concepts for the TFCX design. The plasma heating efficiency and impurity control problews associated with ICRF heating will continue to be tested on PLT.

A portion of the TFTR experimental program will be devoted to the characterization and understanding of high plasma current disruptions. This information, coupled with an improved data base related to lowvoltage startup, would facilitate the design of the IFCX vacuum vessel. 


\section{REFERENCES}

1. Panel III Report to the Magnetic Fusion Advisory Comrittee on Possible TFTR Upgrade Options, January 10, 1983.

2. Tokamak Fusion Core Experiment: Design Studies Based on Subcrconducting and Hybrid Foroidal Field Coils, ORNL/FEDC-83/13, ORivL, FEDC, to be published. 


\title{
4. CONFIGURATION FEATURES
}

Two candidate options were selected for preconceptual design during FY 1983. These include an all-superconducting TF coil configuration (TFCX-S; and a hybrid superconducting-copper TF coil system with watercooled coils located in the shield region in the plane of the TF coils. The removable torus sectors between the TF coils contain no copper coils (TFCX-H).

To provide for practical assembly and waintenance, a number of principles have evolved fron the early reactor studies that have significantly influenced the current tokamak configuration. The first is that of accessitility: all swstems and subsystems must be designed so that easy access is provided to the device and to each major system within the device. A second guiding principle is the concept of modularily: the major systems and components of the device are assembled from modular pieces. Together, these two items - accessibility and modularity - can liad to design approaches for the tokamak that provide the practical capability to service and repair 111 systems and components. In the design process, it is essential that important trade-off studies are performed. It is now widely recognized that the tokamak configuration must be designed from the oltset to be maintained; it is equally important that all of the many key systems be designed as integrated pieces of the totai reactor. The systems integration aspect of the design cannot be overlooked. There are a few key systems that dominate the tokamak configuration, as indicated in Table 4.1. It is primarily these systems that must be designed for practical maintenance.

Table 4.1. Key systems that dominate tokamak configuration designs

\author{
TF ccils \\ PF coils \\ Magnet systems structure \\ Tcrus stricture \\ Vacuum systems \\ impurity control systems
}


A third principle is the desirability of providing hands-on maintenance capability at the device perimeter within a day following shutdown. Hands-on maintenance capability at the shield boundary is presently provided by designing the shielding to limit the dose rate to $2.5 \mathrm{mrem} / \mathrm{h}$ approximately one day after shutdown. These contact operations are valuable for visual inspections, troubleshooting, equipnent setup, and calibration, minor repairs, and supervision of equipment instailation or modifications.

In designing the tokamak configuration to achieve the desired goal and satisfy the preceding principles, the key systems of Table 4.1 dominite the design and have received considerable design emphasis in recent tokamak reactor studies. A comparison of the configuration features from a number of recent fusion reactor studies performed by many different design teams (including international contributions through INTOR) suggest a number of desirable engineering features. These desirable features renresent the present best tokamak design configuration, not only for a power reactor, but also for the nextgeneration tokamak.

These desirable configuration features have been incorporated into the TFCX design, including:

- Relatively few superconducting TF coils. (Designs with 12 to 16 coils are being assessed; since the major radius of these designs is smaller than for the FED/INTOR class, the edge ripple considerations tend toward a slightly larger number of TF coils.)

- A torus consisting of modular sectors radially inserted bet een the outboard legs of the TF coils

- All PF coils located external to the TF coil bore and accessible

- A single, combined vacuum boundary between the plasma chamber and the superconducting TF coils

- Horizontal access at the midplane of the device

- Simplificd gravity support for the torus shield and vacuum system 
An elevation view of TFCX-S is given in Fig. 4.1 and a plan view is given in Fig. 4.2. An elevation view of TFCX-H is given in Fig. 4.3.

In this design, the entire blanket and first wall, together with essentially all of the bulk shield, can be removed by individual extraction of each torus sector using simple, linear, radial motion. Each torus sector is designed to be highly modular with plug-in auxiliaries, such as heating, testing, and impurity control. A combined vacuum boundary is employed in this design; one side of the boundary is the plasma, the other is the magnet cryostat. The vacuum bountary for the removable shield sector is at the outer surface of the sector, which is sealed to the cryostat after the sector is inserted into the torus. The $P$ coils are located to permit removal for maintenance by simple vertical motion. Eact. - the Tr coils can be removed for repair or replacement, if necessary. A TF $c$ ' is removed by removing the associated torus sectors, vertically removing the vessel dome and the upper PF coils, laterally moving the selected TF coil radially outward a small distance, and then vertically lifting the TF coil out of the device.

Key parameters for a baseline all-superconducting TF design (designated TFCX-S) and a baseline superconducting-hybrid TF design (designated TFCX-H) are given in Table 4.2. Since no selection will be made of the preferred TFCX option until FY 1984, the studies have examined variations from the baseline cases. The two baseline cases given have been used as the benchmark for system and component design studies. However, lintil the total preconceptual design effort is completed, many of these variations still represent viable candidate design options.

The TFCX-S baseline design is ignited and employs superconducting TF coils with a maximum field at the winding of $10 \mathrm{~T}$. The device has a major radius of $3.75 \mathrm{~m}$, a minor radius of $1.07 \mathrm{~m}$, a plasma current of $7.7 \mathrm{MA}$, a fusion power of $230 \mathrm{MW}$, a neutron wall loading of $0.9 \mathrm{MW} / \mathrm{m}^{2}$, and sufficient volt-seconds to provide $300 \mathrm{~s}$ of burn.

The TFCX-H baseline design is ignited and employs superconducting TF coils with a maximum field at the winding of $8 \mathrm{~T}$ complemented by water-cooled copper coils located in the shield region to enhance the field on-axis. The device has a major radius of $3.60 \mathrm{~m}$, a minor radius 


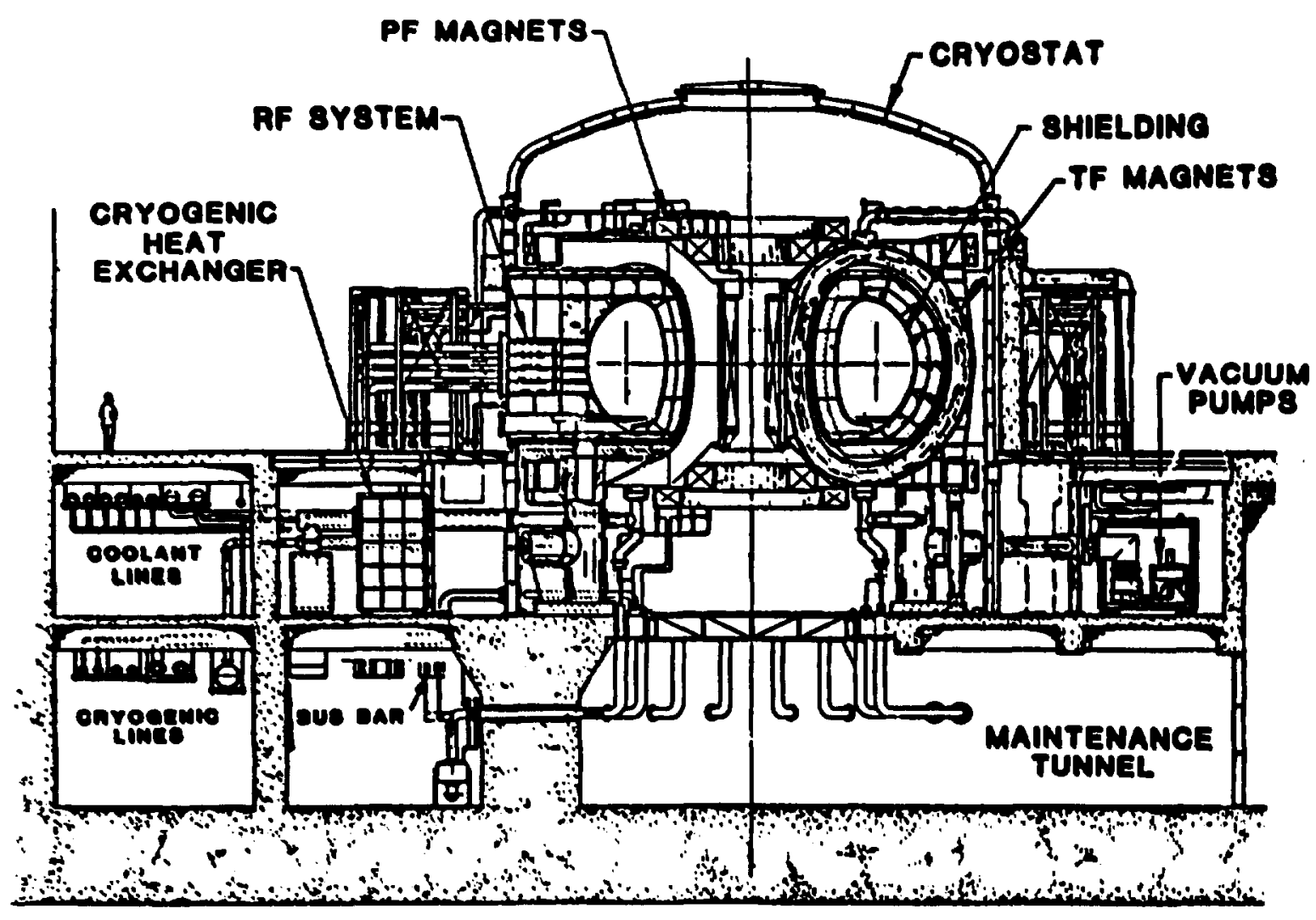

$\dot{5}$

Fig. 4.1. TFCX-S elevation view. 


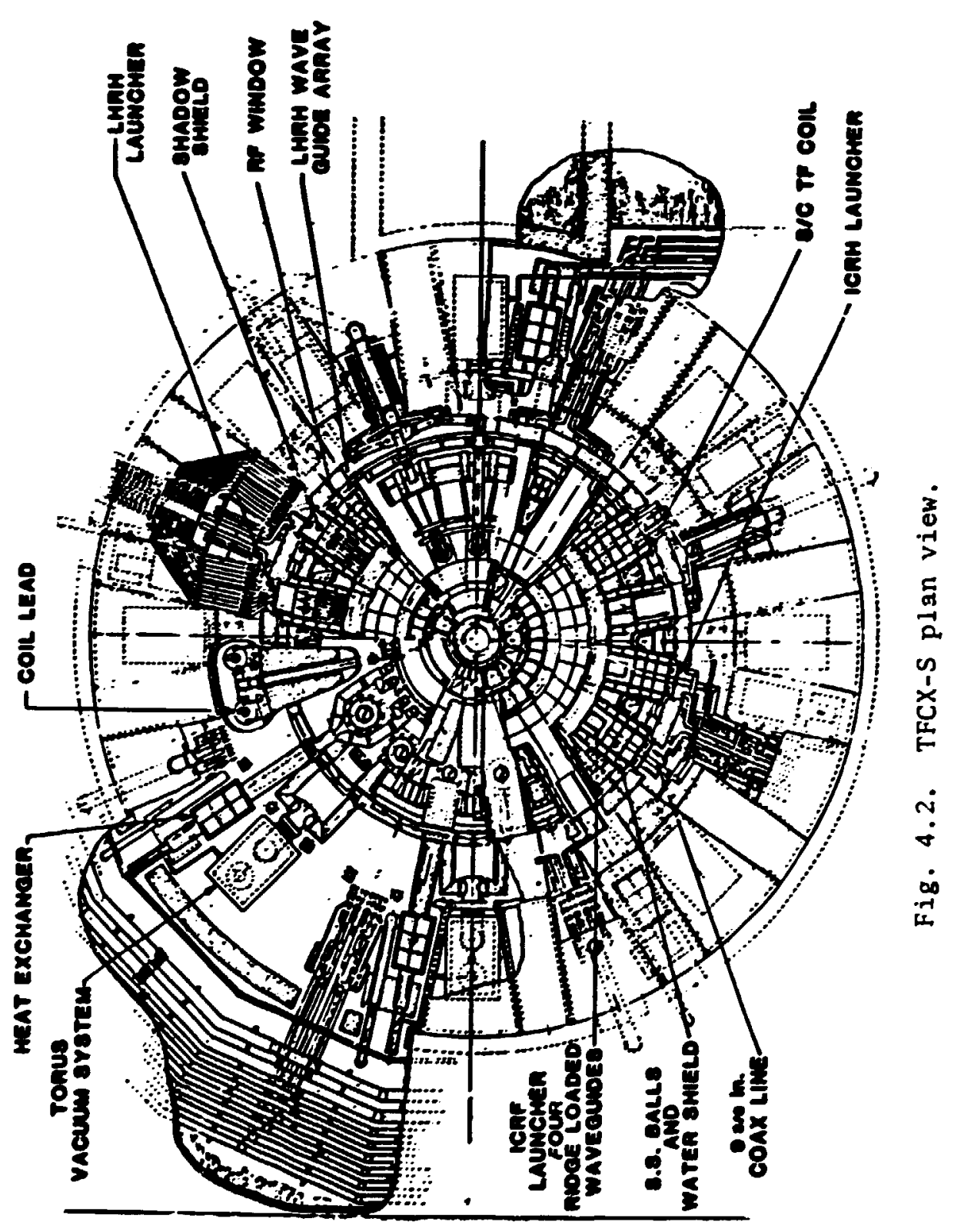




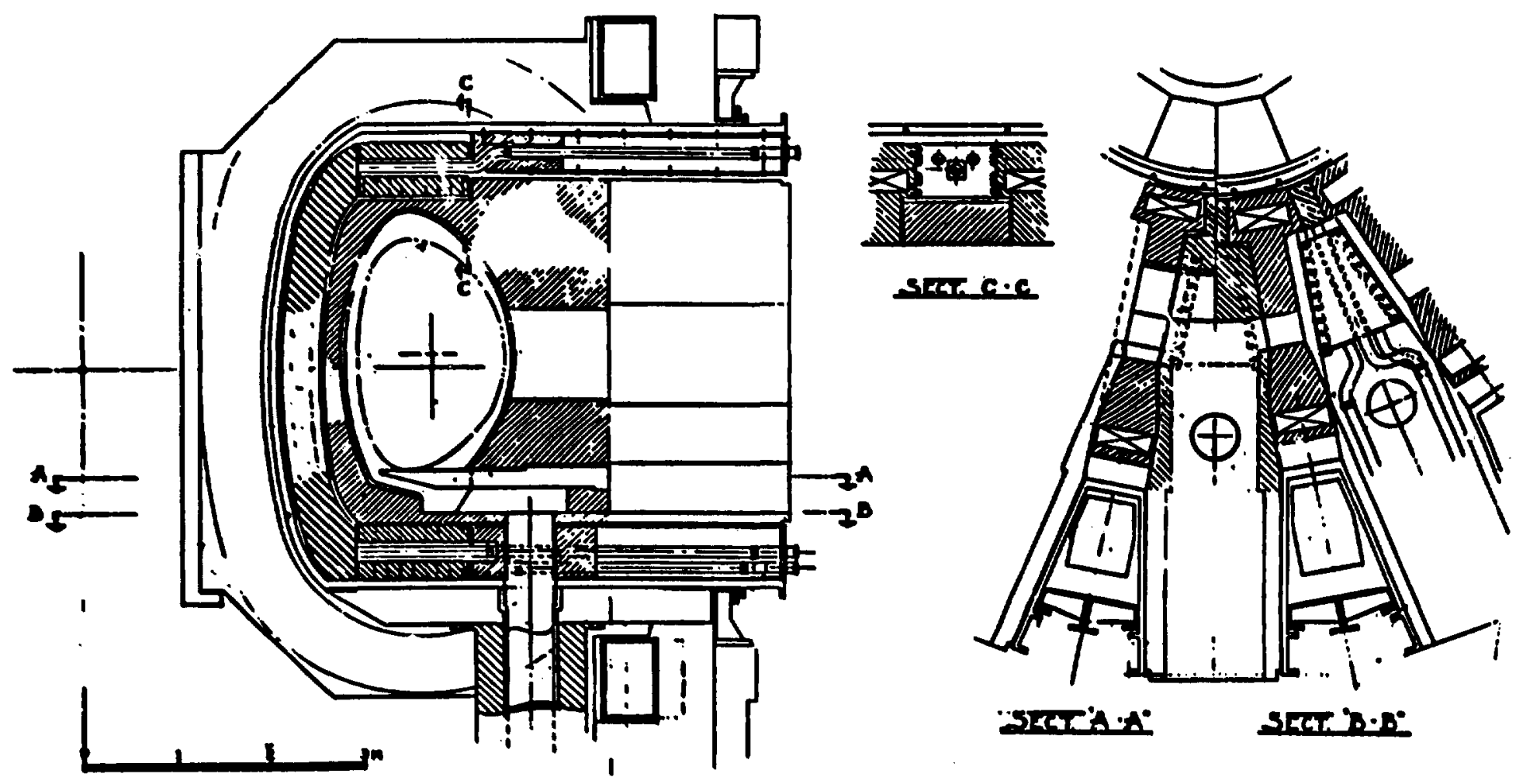

$\hat{a}$

Fig. 4.3. Elevation and plan view schematics of TFCX-H. 
Table 4.2. Key parameters for TFCX-S and TFCX-H

\begin{tabular}{|c|c|c|}
\hline Parameter & TFCX-S & TFCX $-\mathrm{H}$ \\
\hline Major radius, R (m) & 3.75 & 3.60 \\
\hline Plasma radius, a (m) & 1.07 & 0.97 \\
\hline Elongation, $K$ & 1.6 & 1.6 \\
\hline Triangularity, $\delta$ & 0.3 & 0.3 \\
\hline Aspect ratio & 3.5 & 3.7 \\
\hline $\begin{array}{l}\text { Average ion temperature, } \\
\left\langle i_{i}\right\rangle(\mathrm{keV})\end{array}$ & 13.0 & 13.0 \\
\hline $\begin{array}{l}\text { Average electron tempscature, } \\
<\mathrm{T}_{\mathrm{e}}^{>}(\mathrm{keV})\end{array}$ & 13.0 & 13.0 \\
\hline $\begin{array}{l}\text { Average electron density, } \\
\quad<\mathrm{e}_{\mathrm{e}}>\left(10^{20} \mathrm{~m}^{-3}\right.\end{array}$ & 1.9 & 1.9 \\
\hline $\begin{array}{l}\text { Maximum field at } s / c \text { winding, } \\
\mathrm{B}_{\max }(\mathrm{T})\end{array}$ & 10 & 8 \\
\hline Field on-axis, $\mathrm{T}_{t}$ & 4.3 & 4.8 \\
\hline Plasma current, $I_{p}(M A)$ & 7.7 & 4.8 \\
\hline Safety factor, $q_{\psi}$ & 2.0 & 2.0 \\
\hline Beta, B $(\%)$ & 5.9 & 5.6 \\
\hline Fusion power (MW) & 230 & 250 \\
\hline Neutron wall loading $\left(\mathrm{MW} \cdot \mathrm{m}^{2}\right)$ & 0.9 & 1.1 \\
\hline Ignition parameter & 1.0 & 1.0 \\
\hline
\end{tabular}




$$
\text { 4-8 }
$$

of $0.97 \mathrm{~m}$, a plasina current of $7.2 \mathrm{MA}$, a fusion power of $250 \mathrm{MW}$, a neutron wall loading of $1.1 \mathrm{MW} / \mathrm{m}^{2}$, and sufficient volt-seconds to provide $300 \mathrm{~s}$ of burn. 


\section{PLASMA OPERATING SCENARIO}

The piasma operating scenario involves the sequence of operations to initiate and execute a long-pulse discharge cycle in TFCX. Since one of the major objectives of TFCX is to explore the physics and engineering of long-pulse equilibrium burn conditions, means of obtaining long-pulse burn are being investigated both theoretir ' . nd experimentally.

Earlier designs, such as FED/INTOR, wnich were based on inductive current startup and maintenance, had the plasma burn phase limited by the poloidai flux capability. In FED, only about 5\% of the total flux capability was available to maintain the plasma current for the $100-s$ burn; most of the total flux capability was expended in establishing the plasma current. The pulsed nature of an inductively driven tokamak tend; to impose serious limitations on the lifetime, availability, and economy of a tokamak reactor. Therefore, it is highly desirable to achieve a significant level of noninduci.ive current drive in TFCX.

Recent demonstrations of LHCD have been successful in PLT, Alcator-C, Versator II, JIPPT-II, etc., at modest plasma densities and support the proposed current-drive scenario. A successful radio frequency ( $r f$ ) current drive at low density does not necessarily imply an indefinite burn phase at the required density (about $10^{14} \mathrm{~cm}^{-3}$ ). However, such capability will allow application of a large fraction of the $\mathrm{OH}$ induction flux capability to the burn phase at high density. This will facilitate for long-burn-pulse capability while reducing the size of the $\mathrm{OH}$ solenoid.

A reference plasma operating scenario based on LHCD has been developed for TFCX. Table 5.1 summarizes the scenario.

A typical TFCX plasma discharge cycle involves prefill and preionization, current initiation and current drive to full current, bulk heating, burn, plasma shutdown, and pumpdown. The key features of these phases are discussed below with an emphasis on what happens in each phase to better understand the context of the design. 
Table 5.1. TFCX plasma operating scenario (D-T operation)

\begin{tabular}{|c|c|c|c|c|c|c|}
\hline \multirow[b]{2}{*}{ Phase } & \multirow[b]{2}{*}{$\begin{array}{l}\text { Interval } \\
(\mathrm{s})\end{array}$} & \multirow[b]{2}{*}{$\begin{array}{l}\text { Heating/fueling } \\
\text { requirements }\end{array}$} & \multicolumn{4}{|c|}{ Plasma sharacteristics } \\
\hline & & & $\left(\times 10^{13} \mathrm{~cm}^{-3}\right)$ & $\begin{array}{c}\text { Temperature } \\
(\mathrm{keV})\end{array}$ & $\begin{array}{l}\text { Current } \\
\text { (MA) }\end{array}$ & $\begin{array}{l}\text { Power } \\
\text { (MW) }\end{array}$ \\
\hline Prefill chamber & & $\begin{array}{l}\text { Pressure }>10^{-5} \text { torr } \\
\text { from } \sim 10^{-6} \text { torr }\end{array}$ & & & & \\
\hline Current initiation & 0 to $\sim 1.0$ & ECRHI power $\sim 1 \mathrm{MW}$ & $\sim 1$ & $\sim 0.1$ & 0 & 0 \\
\hline Current ramp-up & $\sim 1$ to 30 & $\begin{array}{l}\text { LHRH power } \sim 12 \mathrm{MW} \\
\text { Fuel }\end{array}$ & $\sim 3$ & $\sim 1.0$ & $\sim 6$ & 0 \\
\hline Bulk heating & 30 to 40 & $\begin{array}{l}\text { ICRH power }<40 \mathrm{MW} \\
\text { Fuel } \\
\text { Pump limiter }\end{array}$ & $\sim 12$ & 13.0 & 7 & $\sim 250$ \\
\hline Burn & 40 to 340 & $\begin{array}{l}\text { ICRH power off } \\
\text { Fuel } \\
\text { Pump limiter }\end{array}$ & $\sim 12$ & 13.0 & 7 & $\sim 250$ \\
\hline Shutdown & 340 to 360 & $\begin{array}{l}\text { Fuel off } \\
\text { Particle exhaust }\end{array}$ & $\sim 2$ & $\sim 1.0$ & 0 & 0 \\
\hline Pumpdown & 360 to 390 & Pumpdown pressure & & & & \\
\hline
\end{tabular}




\subsection{PREFILL AND IONIZATION}

About $1 \mathrm{MW}$ of extraordinary mode, electron cyclotron res.nance frequency wave at about 100 to $110 \mathrm{GHz}$ is launcheü from the high-field side for a duration of $\sim 1$ s to preionize and heat the electrons. The heated electrons ( $\mathrm{T}_{\mathrm{e}} \sim 100 \mathrm{eV}, \mathrm{n}_{\mathrm{e}} \sim 1 \times 10^{23} \mathrm{~cm}^{-3}$ ) are expected to be located over a radial width of $\sim 0.4 \mathrm{~m}$. This permits the application of a low toroidal loop voltage $\left(\mathrm{v}_{1}<25 \mathrm{~V}\right)$ to initiate and rampup the plasma current.

To effect proper penetration and absorption of the wave, it must come from the high-field side of the plasma with the extraordinary mode, which means the electric field is perpendicular to the toroidal field. An injection angle of $85^{\circ}$ is specified but needs substantiation when the exact startup plasma parameters are known.

\section{S.: CURRENT INITIATION AND RAMP-UP}

The baseline operating scenario calls for an all rf stertup saving the $\mathrm{OH}$ transformer volt-seconds for the long-pulse burn. Currentdrive techniques presentir in use indicate a decrease in efficiency (amperes of plasma current driven to watts of LHRH power) as the density is increased. Hence, LHRH is used during the low-density parts of the startup and into the bulk heating period until the density reaches a level where LHRH becomes less effective; the $\mathrm{OH}$ transformer is then switched on.

Either D, H, or D-T gas can be used with current drive. For ignited conditions in D-T operation, a startup time of $\sim 30 \mathrm{~s}$ is used in the baseline scenario. To size the LHRH system for current drive, a range of 0.5 to $7.2 \mathrm{MA}$ was used resulting in a power requirement of about 12 to $14 \mathrm{MW}$. This power level should be controllable over a 100-dB range. The frequency is twice the lower hybrid frequency to avoid ion interaction. The frequency is $\sim 2.7 \mathrm{GHz}$.

\subsection{BULK HEATING}

About $40 \mathrm{MW}$ of ICRF power is applied at a frequency of about $70 \mathrm{MHIz}$ for about $10 \mathrm{~s}$. The plasma temperature increases from $\sim 1$ to $\sim 13 \mathrm{keV}$ 
and plasma density increases to a full density of $\sim 1.2 \times 10^{14} \mathrm{~cm}^{-3}$ at full plasma current of $\sim 7$ MA via pellet fueling and gas puffing, maintaining nearly equal deuterium and tritium content. The contact between plasma and limiter is controlled to achieve adequate particle exhaust through the limiter channels without overheating the leading edge of the pump limiter. The value of $q_{\psi}$ at the plasma edge is maintained at 2.0 during this phase. Under the given plasma beta and confinement assumptions, ignition is achieved and the fusion power is expected to reach $\sim 250 \mathrm{MW}$.

\subsection{BURN}

Once the full plasma conditions of temperature, density, and current are achieved, ignition is obtained and an operating point established. The $O H$ transformer flux capability is then used to maintain the placm $z$ current during the 300-s burn. The pump limiter is assumed to exhaust the helium and control the plasma edge adequately, but auxiliary impurity control or reduction schemes may be needed. An attractive possibility for use in conjunction with a pump limiter is the maintenance of a radiation-cooled plasma edge, which is expected to minimize impurity production and ingestion at the plasma edge. Assuming adequate helium. exhaust and impurity control, the plasma burn time is limited either b: the volt-second capability of the $\mathrm{OH}$ coils or by significant resistive diffusion of the plasma poloidal flux. With nearly classical toroivia: plasma conductivity, the latter time scale is estimated to be a few hundred seconds.

An option at the end of the burn would be to recharge the $\mathrm{OH}$ transformer, reduce the temperature and density of the plasma, and maintain the current at or near its full value with the LHRH system and then to initiate another $300-s$ burn pulse by applying the ion cyclotron resonic heating (ICRH) to raise the plasma temperature, bring up the plasma density, and use the of transformer flux capability to obtain another 300-s burn. 


\section{SYSTEMS STUDIES}

During the last year, a large variety of potential concepts for what the TFCX device should be have been examined from a global systems point of view. These studies tend to be ongoing and rely heavily on the use of the FEDC systems code. This ccie has been developed over the last five years. It is used to calculate the tokamak configuration, performance, and cost as a function of plasma and engineering parameters. The code has a modular structure to allow independent modeling of each major tokamak system or component. Each module incorporates the latest design thinking in the representation of each major system or component.

The more recent systems studies have coupled the systems code to available MHD and magnetic field strength analysis codes in order to ensure that plasma and magnet systems constraints are satisfied.

The adequacy of the PF system (based on the constraints of plasma shape, burn time, maximum field at the PF coil, and configurational constraints) was determined by iterative execution of the FEDC systems code, an MHD equilibrium code, and EFFI (a code to svaluate magnetic field strength). Figure 6.1 is a flow diagram of the procedure.

The following sections present results of a number of the major systems studies performed during this period.

\subsection{DRIVEN-CURRENT TOKAMAK (DCT) SCOPING STUDIES}

A series of superconducting Driven-Current Tokamak (DCT) devices were examined early in FY 1983 by FEDC and by PPPL. This scries of devices range from a PLT-size plasma device (denoted DCT-2) through an ignition-size D-T device (denoted DCT-8). The smallest device is distinguished by the choice of a IF coil size comparable to that of the Large Coil Test Facility (LCTF). The largest device is about $70 \%$ the size of FED.

The DCT-2 device is designed solely for long-pulse hydrogen plasma operation and involves no D-T plasma operation. As such, the role of DCT-2 in the fusion program would be to develop the nonnuclear technology and physics required for an Engineerirg Test Rear,tor (ETR). 


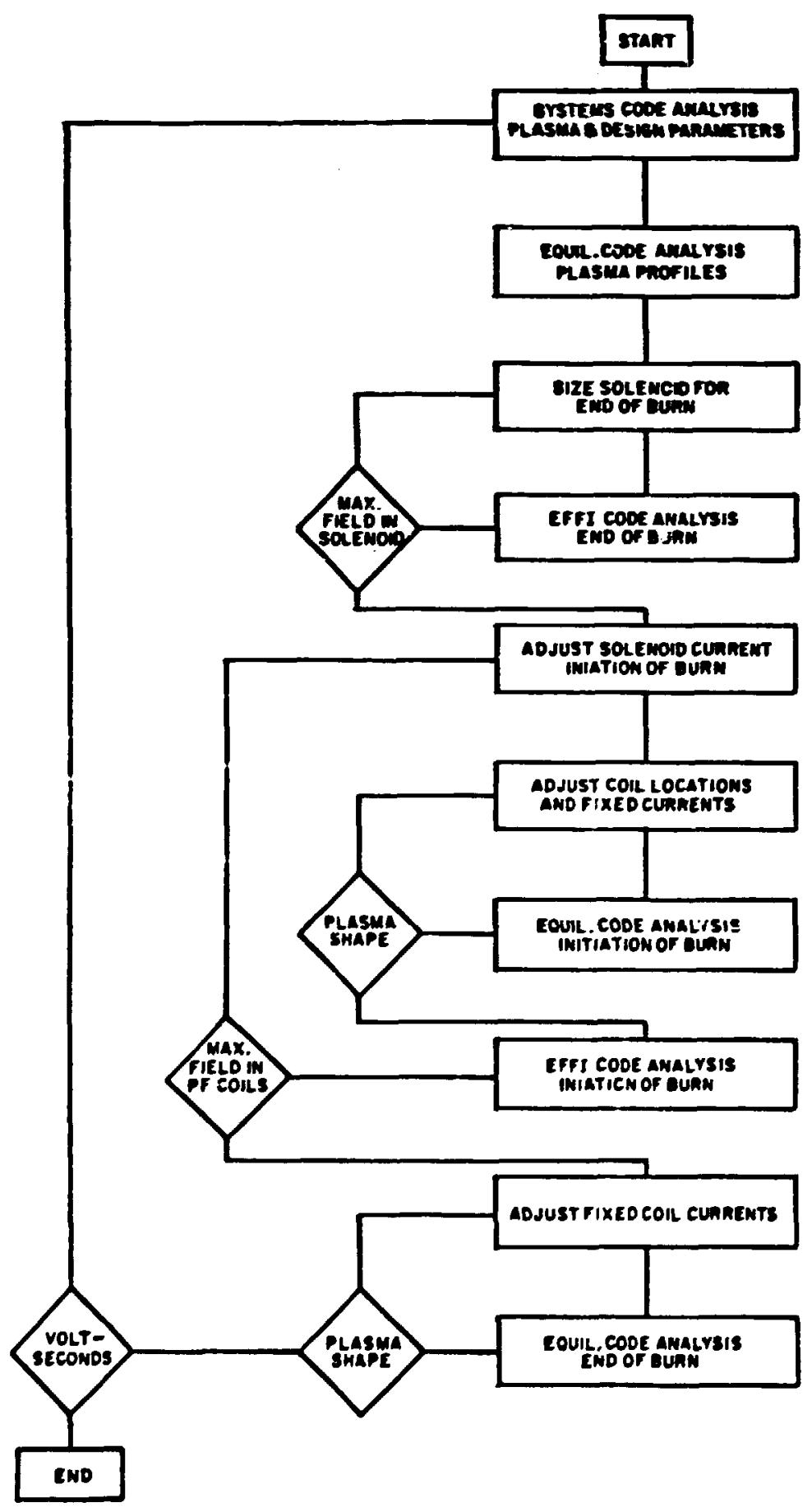

Fig. 6.1. Flow diagram of system study procedure. 
The DCT-8 is an ignited, long-pulse D-T device. The distinctive feature of one option for DCT-8 is to use a TF coil system in which both superconducting and copper coils are used; this facilitates high-field, high-performance operation at reduced size and cost. The objectives for this device combine the long-pulse objectives of a superconducting tokamak with the nuclear technology and physics objectives of a D-T burning experiment. It should be noted that the designation of DCT-8 was changed to TFCX-H later in the year.

The major device parameters for the DCT-2 and DCT-8 are listed in Table 6.1. These parameters are representative of on!y one design point in the available design space for each device; trade-offs in many of these parameters typically occur during the preconceptual design to identify the cost/performance sensitivities and thereby permit iteration to a final set of parameters.

\subsection{DCT-\& CONFIGURATION TRADE STUDY}

Four hybrid configuration options ware identified as possible candidate designs for the DCT-8 device (see Fig. 6.2). Hybrid configuration options included locating copper TF coils: (1) in the shield, both under the TF coil and in the removable shield module; (2) in the shield region, solely under the TF coil; (3) solely in the removable shield module; and (4) as an integrated entity. An all-superconducting option (Case 1 ITFCX-S) was specified as the candidate option for comparison with the selı ited hybrid option.

A scoping study was made to reduce the candidate hybrid options down to one approach to compare it with the all-superconducting design. In evaluating the hybrid options, locating a liquid-nitrogen-cooled copper coil with the superconducting TF coil (Case 5) was found to raise the overall reactor cost when compared with Cases 2 through 4 . This resulted from the lower-field contribution of the copper, being located further from the plasma, and the inability of the copper/superconducting arrangement co be configured with a variable inboard-to-outbioard cross section. Support for the copper coils in the Case 2 arrangement appeared to be quite difficult; therefore, it was dropped as a candisate hybrid 
Table 6.1. Key parameters for DCT-2 and DCT-8

\begin{tabular}{lll}
\hline & DCT-2 & DCT-8 \\
\hline Major radius (m) & 2.9 & 3.6 \\
Plasma radius (m) & 0.77 & 0.75 \\
Plasma elongation & 1.6 & 1.6 \\
Fusion power (MW) & 0 & 390 \\
Neutron wall loading (MW/m $\left.{ }^{2}\right)$ & 0 & 2.2 \\
Heating (MW) & & 23 \\
$\quad$ ICRH & 0 & 10 \\
LCRH & 0 & 00 \\
Amplification factor, Q & 3.0 & 4.3 \\
Average total beta (\%) & 4.0 & 5.9 \\
Plasma current (MA) & & \\
TF coil clear bore, & $2.6 \times 3.6$ & $3.6 \times 5.2$ \\
width $\times$ height (m) & & \\
Cu coil clear bore, & NA & $3.0 \times 3.9$ \\
width $\times$ height (m) & 4.7 & 7.0 \\
Field on -axis (T) & & \\
\hline
\end{tabular}




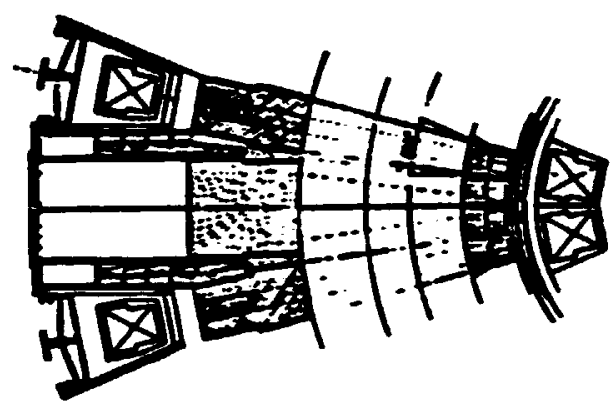

\section{CASE 2 - CU IN SHIELO UNDER TF COIL AND IN REMOVABLE MODULE}

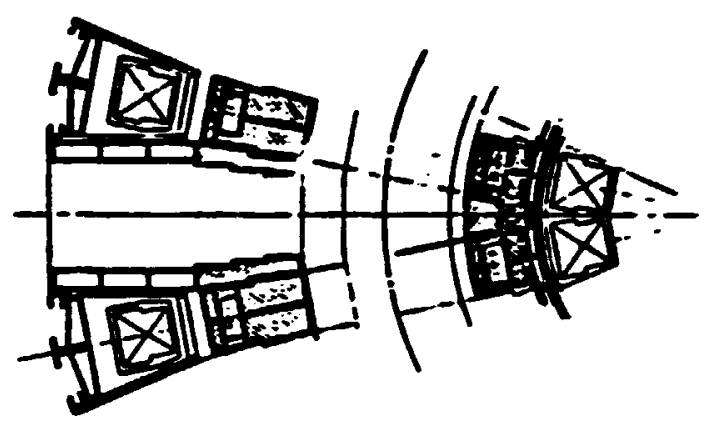

\section{CASE 3 - CU IN SHIELD UNDER TF COIL}

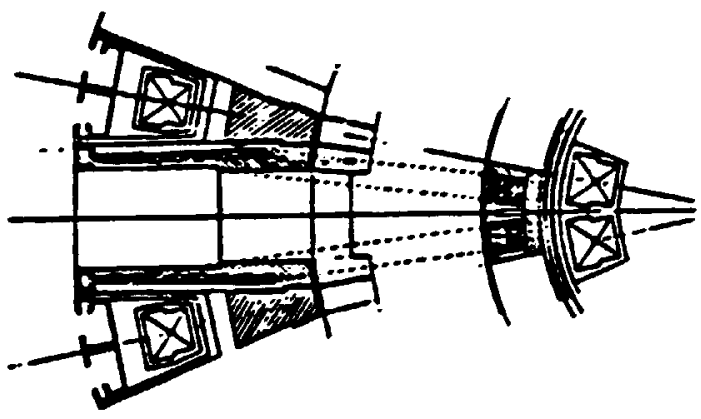

\section{CASE 4 - CU IN SHIELD IN}

AEMOVABLE MOdLLE

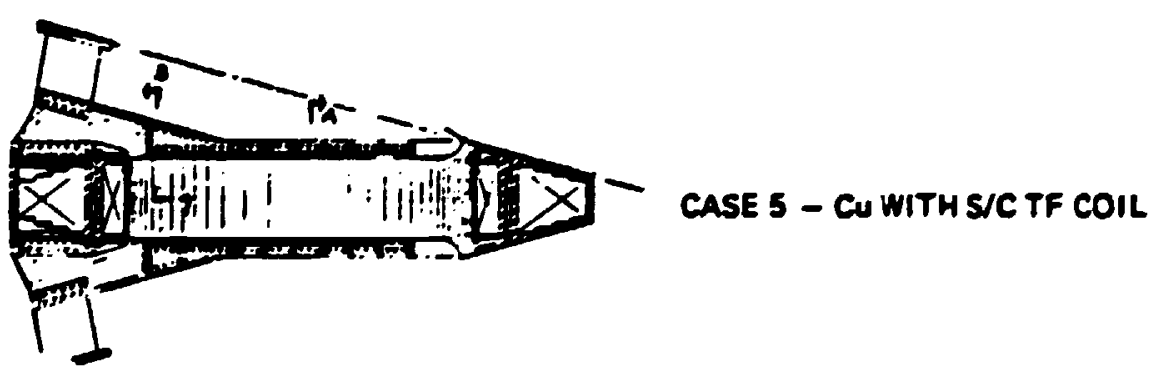

Fig. 6 2. DCT configuration options. 
Locating the copper coils entirely in the shield (Case 4) offered better access to maintain the copper coils and to supply cooling and electrical hookup compared to Case 3; hovever, supporting the copper coils against their out-of-plane forces appeared to be more difficult. A possible support method for Case 4 was to provide external reinforcenent at the outside of the vessel. The support nethod for Case 3 occurs entirely inside the shield, independent of the removable shield nodule. The intercoil support structure is attached at the point where the out-ofplane forces appeared to be more difficult. A possible support method for Case 4 was to provide external reinforcement at the outside of the vessel. The support method for Case 3 occurs entirely inside the shield, independent of the removable shield module. The intercoil support structure is attached at the point where the out-of-plane magnetic loads are being app!ied, rather than relying on the removable shield module to carry the loads in torsion to an external support structure. For this reason, Case 3 was selected as the hybrid copper/superconducting option for further design definition and, ultimately, to be compared with an all-superconducting option (Case 1) and to an all-copper device. (Note: Case 3 of this series ultimately was designated TFCX-H and Case 1 became TFCX-S.)

\subsection{SYSTEMS RESULTS FOR TFCX-S AND TFCX-H}

System studies performed on a self-consistent basis require

- a well-defined configuration con':ept,

- specified design guidelines and criteria from which design points can be derived, and

- reasonable models by which performance and operating characteristics can be assessed.

The TFCX-S and TFCX-H design points were derived based upon the design guidelines and criteria listed in Table 6.2. Other than the field enhancement due to the copper insert, the design criteria for the reference designs differ only in the TF coil technology selected for each device. 
Table 6.2. TFCX design guidelines and criteria

\begin{tabular}{ll}
\hline Parameter & Value \\
\hline Elongation & 1.6 \\
Triangularity & 0.3 \\
Safety factor & 2.0 \\
Ignition parameter & 1.0 \\
Maximum ourn time ${ }^{a}$ (s) & 300 \\
Cumulative burn time (s) & $2 \times 10^{5}$ \\
Maximum TF ripple (b) & 1.5 \\
Plasma temperature (keV) & 13 \\
Startup current maintenance & LHRH \\
Maximum field at PF coils (T) & 8 \\
Maximum field at TF coils (T) & $8-10$ \\
Winding cavity current density in TF coils & \\
(A/cm ${ }^{b}$ & $2600-2000$ \\
Field enhancement on-axis (T) & $0-1.6$ \\
Allowable TF dose to insulator (rad) & $10^{9}$ \\
Allowable TF nuclear heating (mW/ $\mathrm{cm}^{3}$ ) & 1.00 \\
Inboard & 0.25 \\
Outboard & 1.0 \\
Shutdown dose rate (mrem/h) & 24 \\
Cooldown time (h) & \\
\hline
\end{tabular}

$a_{\text {Inductive driven. }}$

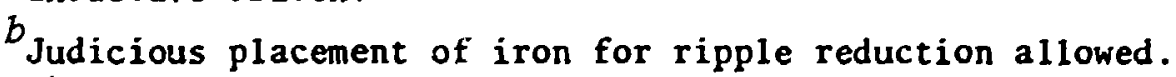


There are several distinctions between the early DCT scoping studies and the more recent follow-on system studies. When the early DCT studies were conducted, a well-defined, viable hybrid configuration concept had not been developed. Thus, the distinctions between hybrid and superconducting configurations were not reflected in the DCT study results. In the interim between the two efforts, the FEDC systems code was substantially upgraded and updated. For the more recent studies, the procedure illustrated in Fig. 6.1 was used. The procedure involves iterative execution of three major codes: the FEDC systems and MHD equilibrium codes and EFFI, a code to evaluate magnetic field strength. All three codes are required to ensure that the constraints of the plasma shape, burn time, and maximum field at the PF coils are simultaneously met.

Using the design guidelines and criteria (Table 6.2) and the indicate 3 methodology (Fig. 6.1), design points were established (1) for two configurations using only superconducting TF coils (at maximun field strengths of 8 and $10 \mathrm{~T}$, respectively) and (2) for two configurations using superconducting TF coils supplemented with copper TF coil inserts in the shield sectors (with maximum field strengths of $\delta$ or $10 \mathrm{~T}$, respectively, for the superconducting coils). The key parameters are given for these four cases in Table 6.3.

As indicated in Table 6.3, the devices with maximum field at the superconducting coils of $8 \mathrm{~T}$ are smaller than their 10-T counterparts, and the superconducting devices are smaller than their hybrid counterparts. Contributory factors that lead to these results are described in the following:

1. The higher-field devices have smaller plasma radii. This contributes toward a decrease in radial build; howcver, it is offset by more subtle changes in the following areas.

2. The higher-field devices require more volt-seconds during burn due to a smaller plasma cross section which tends to enlarge the $0 \mathrm{H}$ bore.

3. The higher-field devices require thicker inboard shields (due to higher wal: loading) and thicker TF_coils (if $10 \mathrm{~T}$ vs $8 \mathrm{~T}$ ).

4. Thicker inboard shields and thicker TF coils not only make the device larger but increase the distance from the shaing coils 
Table 6.3. Key parameters .or TFCX design options

\begin{tabular}{|c|c|c|c|c|}
\hline Parameter & $S / 8 T^{a}$ & $\mathrm{~S} / 10 \mathrm{~T}$ & $H / 8 T$ & $\mathrm{H} / 10 \mathrm{~T}$ \\
\hline Major radius (m) & 3.55 & 3.75 & 3.60 & 4.38 \\
\hline Minor radius $(m)$ & 1.45 & 1.07 & 0.97 & 0.82 \\
\hline Aspect ratio & 2.44 & 3.51 & 3.71 & 5.35 \\
\hline $\begin{array}{l}\text { Bucking cylinder inner } \\
\text { inner radius (m) }\end{array}$ & 0.74 & 0.86 & 1.12 & 1.62 \\
\hline $\begin{array}{l}\text { OH current (at beginning } \\
\text { of burn) }(\xi)\end{array}$ & -91 & -83 & 0 & +42 \\
\hline Volt-seconds required $(v-s)$ & 12.6 & 17.1 & 18.9 & 25.5 \\
\hline Plasma current (MA) & 10.8 & 7.7 & 7.2 & 5.8 \\
\hline PF system (MAT) & 68 & 79 & 78 & 85 \\
\hline TF/CI ${ }^{b}$ (MAT) & $46 / 0$ & $81 / 0$ & $63 / 24$ & $121 / 34$ \\
\hline ICRH power (MW) & 15 & 24 & 25 & 43 \\
\hline Field on-axis (T) & 2.62 & 4.34 & 4.84 & 7.10 \\
\hline Beta & 0.085 & 0.059 & 0.056 & 0.039 \\
\hline Electron density $\left(10^{14} \mathrm{~cm}^{-3}\right)$ & 0.52 & 1.00 & 1.17 & 0.39 \\
\hline Energy confinement time (s) & 3.75 & 1.97 & 1.68 & 1.14 \\
\hline Neutron wall load $\left(M W \cdot m^{-2}\right)$ & 0.3 & 0.9 & 1.1 & 2.1 \\
\hline Fusion power (MW) & 110 & 230 & 250 & 490 \\
\hline
\end{tabular}

a Notation $S / 8 \mathrm{~T}$ refers to "all-superconducting" TF coils/maximum field at superconducting $(\mathrm{s} / \mathrm{c})$ coils of $8 \mathrm{~T}$; $\mathrm{S} / 10 \mathrm{~T}$ refers to all-superconducting/10 $\mathrm{T}$ at the $\mathrm{s} / \mathrm{c}$ coils; $\mathrm{H} / 8 \mathrm{~T}$ refers to superconducting-copper hybrid TF coils/8 $\mathrm{T}$ maximum field at the $\mathrm{s} / \mathrm{c}$ coils; $\mathrm{H} / 10 \mathrm{~T}$ refers to hybrid TF coils/10 $\mathrm{T}$ at the $\mathrm{s} / \mathrm{c}$ coils.

${ }^{b} \mathrm{TF} / \mathrm{CI} \equiv$ toroidal field coils/copper insert coils. 
to the plasma edge. In the hisher-fitld devices, the plasma edge: is closer to the plasma center of current. As a result, shaping is more difficult, requiring larger shaping coils, which linits the $O H$ swing and tends to enlarge the $\mathrm{OH}$ bore.

Based on these results, it was decided (in agreement with PPPL) to pursue the 10-T all-superconducting point as TFCX-S and the 8-T sup-:conducting/copper hybrid point as TFCX-H. The characteristics of these designs will form the basis for later comparison with a preferred allcopper TF design. 


\section{MAGNETIC SYSTEMS}

The magnetic systems consist of (1) the TF coils; (2) the PF coils, which include the $\mathrm{OH}$ solenoid and equilibrium field (EF) coils external to the TF coil bore; and (3) the cryostat for options involving superconducting coils. The overall arrangement of the magnets is depicted in Fig. 7.1, and the key parameters of the magnets are given in Table 7.1. Each of the systems is briefly described below.

The magnet systems for two superconducting TFCX options are described. In the first option, designated TFCX-S, the required field-on-axis is developed entirely by 16 superconducting TF coils. In the alternative option, designated TFCX-H, the field-on-axis is developed by a combination of 16 superconducting TF coils and 16 copper insert coils located in the plane of and inside the bore of the superconducting coils. The TFCX-H copper insert coils are embedded in the shield modules, and the device may be operated with or without the copper insert coils energized.

\subsection{SUPERCONDUCTING TF COILS}

In the TFCX-S option, the TF coils produce a field-on-axis of $4.3 \mathrm{~T}$ at a major radius of $3.75 \mathrm{~m}$ and develop a maximum field of $9.7 \mathrm{~T}$ at the TF coil. Four different conductor/coolant concepts have been considered for this application. These concepts are discussed separately. Any of the iour will fit within the space envelope allocated to the TF coils and will satisfy the field-on-axis requirement.

In the TFCX-H option, the superconducting coils operate at a peak field level of $\sim 8.0 \mathrm{~T}$ (rather than $\sim 10 \mathrm{~T}$ as in TFCX-S) and alone can produce $3.5 \mathrm{~T}$ on-axis at a major radius of $3.60 \mathrm{~m}$. When the copper coils are energized, the field-on-axis is increased to $4.8 \mathrm{~T}$, whereas the maximum field at the copper coil is $7.6 \mathrm{~T}$. In both options, the ripple in the toroidal field is limited to $1.5 \%$ at the edge of the plasma and $0.1 \%$ at the center of the plasma.

The superconducting coils are designed to be cryostable against the combined heat load of resistive heating, nuclear heating, and eddy current heating. The magnet systems are required to withstand magnetic 


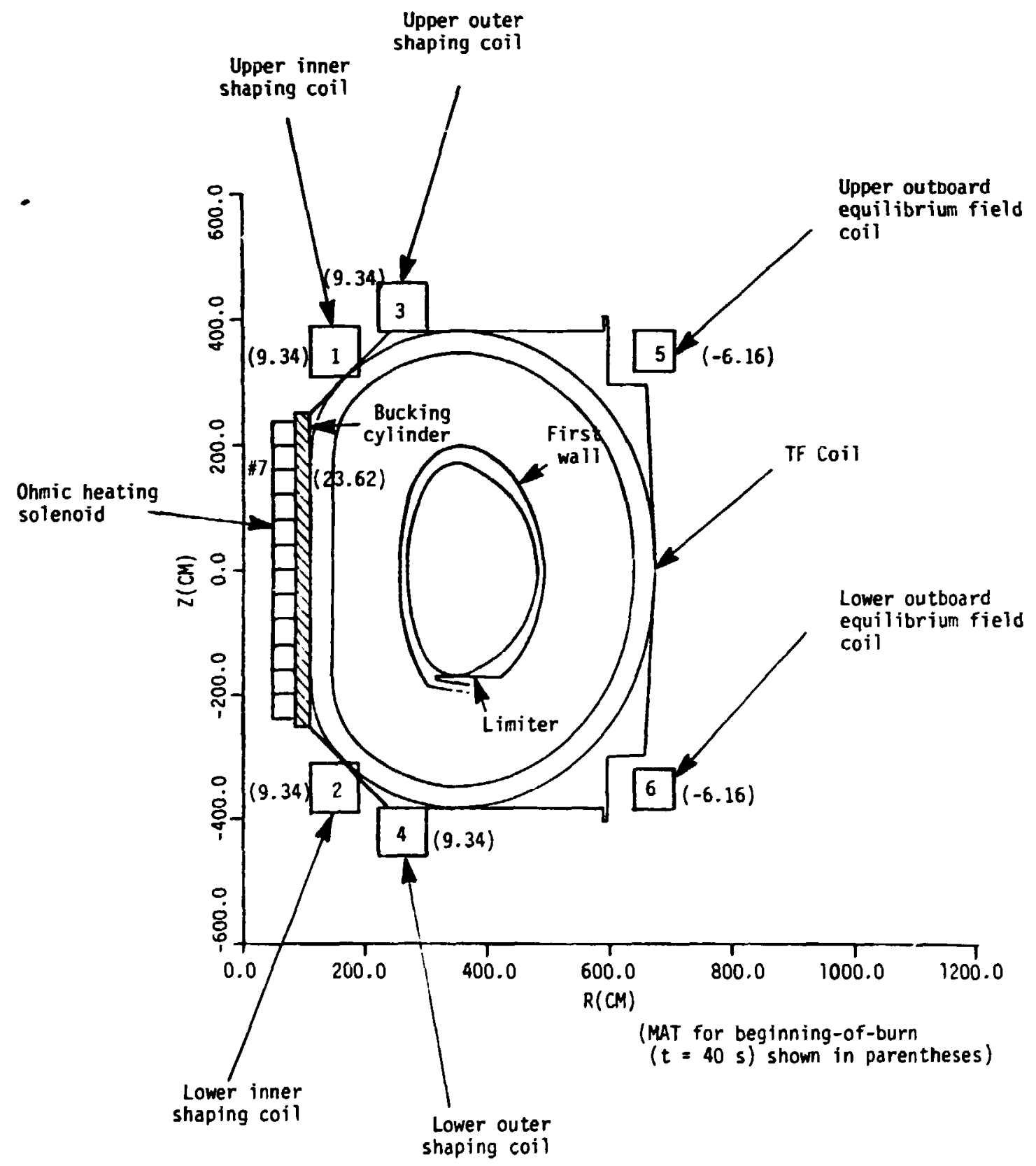

Fig. 7.1. Overall arrangement of the magnets. 
Table 7.1. FED magnetic system parameters (TFCX-S)

\begin{tabular}{|c|c|}
\hline Description & At $10 \mathrm{~T}$ \\
\hline Major axis (m) & 3.75 \\
\hline Field on-axis (T) & 4.3 \\
\hline Number of pulses & $1.1 \times 10^{5}$ \\
\hline \multicolumn{2}{|l|}{ TF coils } \\
\hline Number & 16 \\
\hline Conductor & NbTi forced flow \\
\hline Design field at winding (T) & 10 \\
\hline Winding bore (m) & $6.1 \times 4.2$ \\
\hline $\begin{array}{l}\text { Maximum permissible radiation } \\
\text { dose (rad) }\end{array}$ & $10^{9}$ \\
\hline Ampere-turns per coil (MAT) & 5.06 \\
\hline Overall current density $\left(\mathrm{A} / \mathrm{cm}^{2}\right)$ & 1470 \\
\hline Operating current (kA) & 28 \\
\hline Stcred energy/coil (MJ) & 470 \\
\hline Coil mean perimeter (m) & 18.8 \\
\hline \multicolumn{2}{|l|}{ Bucking post radius (m) } \\
\hline Outside & 1.72 \\
\hline Inside & 1.06 \\
\hline \multicolumn{2}{|l|}{ PF coils } \\
\hline Volt-seconds (Wb) & 17 \\
\hline Conductor & NbTi \\
\hline Maximum field at coil (T) & 8 \\
\hline Charging time (s) & 30 \\
\hline Discnarge time (s) & 30 \\
\hline Length of central solenoid (m) & 6 \\
\hline Winding current density $\left(\mathrm{A} / \mathrm{cm}^{2}\right)$ & 1500 \\
\hline Stored energy in the solenoid (GJ) & 1.0 \\
\hline
\end{tabular}


loads arising during normal and abnormal conditions, as well as dead weight and 1-g horizontal and/or vertical seismic loading, while satisfying a variety of access and clearance constraints.

The functional requirements that must be satisfied by the TF coils are included in the following:

- Inboard scrape-off distance requirements

- Shielding sufficient to limit the nuclear heat generation rate and the displacements per atom (dpa) damage in the inboard leg of the TF coil to an acceptable level

- Space occupied by the vacuum vessel

- Gaps between the vacuum vessel and TF coils and between the vacuum vessel and the shield sufficient to preclude contact during credible seismic events

- Space occupied by the TF coil case, influenced by designallowable working stresses

- TF coil winding space, determined by the ampere-turns needed to satisfy the field-on-axis requirement and by realistically achievable current densities for the conductor concept selected

- Space required by the 'ucking post [The inside diameter is determined by the space requirements of the $\mathrm{OH}$ solenoid, which provides the necessary volt-seconds during startup; the radial build (thickness) is determined by the net centering load on the TF coil and the design-allowable stress for the cylinder material(s).]

The conductor and winding are designed to be cryostable. The maximum heat flux that can be dissipated without the loss of superconductivity of the conductor (critical heat flux) is characteristic of the conductor configuration. The coil is designed so that the actual heat flux resulting from the combined effect of normal resistive heating, eddy current heating, and nuclear heating does not exceed the critical heat flux for the conductor.

Conductors cooled with forced-flow helium cannot, however, be characterized as cryostable in the same sense as the conductor cooled with pool-boiling helium. Force-cooled conductors considered in this 
report are the cable-in-conduit type. Individual strands in such a conductor can go normal due to localized strand movement. An energy deposition tolerance limit for such conductors is generally determined experimentally.

The critical current density (maximum current density at which the conductor remains superconducting) is degraded when a mechanical strain is imposed upon the conductor. The net strain from all sources is usually limited to about $1.5 \%-2 \%$ for NbTi conductors, and to about $0.5 \%$ for $\mathrm{Nb}_{3} \mathrm{Sn}$ conductors.

Structural design and analysis of the T? coils and associated components is in accordance with the structural criteria. These criteria consider potential ductile failure due to gross overloading as well as failure due to growth of a postulated structural defect to critical size under the action of a cyclic (pulsed) load. The structural criteria that address ductile failure provide, for normal loading conditions, a margin of at least 1.5 against formation of a fully plastic section in either membrane loading or bending and a margin of at least 3 against rupture. For abnormal loading conditions, the required margins are twothirds of those for normal loading conditions. The criteria that deal with crack growth considerations provide a margin of at least 3 against critical stress after one design 1 ife and a margin of at least 2 after two design lives.

Maximum allowable discharge voltage is limited to $3000 \mathrm{~V}$ for the magnets cooled with pool-boiling helium. For force-cooled magnets, this limit can be $10,000 \mathrm{~V}$.

The peak temperature rise of the winding in the event of a quench is limited to $200 \mathrm{~K}$. This 1 imit is primarily governed by the thermal stresses in the hot zone of the winding.

Average current density values for the winding pack and for the overall coil cross section have been adopted. These figures depend upon the conductor concept, the peak field at the winding, the winding operating temperature, and the selected cooling option. The current densities used in studies of the present TF coil design are listed in Table 7.2. 


$$
7-6
$$

Table 7.2. TF coil current densities

\begin{tabular}{lccccc}
\hline Conductor & $\begin{array}{c}\text { Peak field } \\
(\mathrm{T})\end{array}$ & $\begin{array}{c}\text { Temperature } \\
(\mathrm{K})\end{array}$ & $\begin{array}{c}\text { Cooling } \\
\text { option* }\end{array}$ & $\begin{array}{c}\text { Current density } \\
\left(\mathrm{A} / \mathrm{cm}^{2}\right) \\
\text { coil } \\
\text { winding }\end{array}$ \\
\hline $\mathrm{Nbti}$ & 8 & 4.2 & PB & 1500 & 2600 \\
$\mathrm{NbTi}$ & 8 & 4.2 & FF & 1500 & 2600 \\
$\mathrm{NbTi}$ & 10 & 1.8 & PB & 1400 & 2000 \\
$\mathrm{NbTi}$ & 10 & 3.0 & FF & 1400 & 2000 \\
$\mathrm{Nb}{ }_{3} \mathrm{Sn}$ & 10 & 4.2 & FF & 1400 & 2000 \\
\hline
\end{tabular}

${ }^{\star} \mathrm{PB}=$ Pool boiling; $\mathrm{FF}=$ Forced flow 
The selection of a TF coil winding insulating material is influenced by the lifetime-integrated radiation dose to which the winding will be exposed, since the mechanical strength of some insulating materials is seriously degraded by nuclear radiation. The maximum achievable current density is influenced by the compressive strength of the insulating material. Glass-reinforced epoxies such as G-10 and G-11 begin to show a marked decrease in strength after about $2 \times 10^{8} \mathrm{rad}$ exposure. It is not cost-effective to provide enough shielding to limit the winding fluence to $2 \times 10^{8} \mathrm{rad}$, nor is it cost-effective to base the winding $\mathrm{d} \cdot:$ ign on the degraded strength of irradiated material. Consequently, use of G-10 or similar insulating materials is not judged feasible for the TF coils if the dose exceeds $2 \times 10^{8} \mathrm{rad}$.

Polyimide insulators have been demonstrated to have superior radiation resistance. In particular, Keramid polyimide, when loaded in compression normal to the plane of the material, has a compressive strength for unirradiated material that is quite competitive with unirradiated G-10CR $(\sim 120 \mathrm{ksi}$ at $77 \mathrm{~K})$; furthermore, within the error bands of the strength measurements, the compressive strength is urchanged for gamma-ray doses up to $10^{10} \mathrm{rad}$. For the TFCX design, the maximum fluence on the TF coils is about $6 \times 10^{8} \mathrm{rad}$; consequently, Keramid polyimide is specified as the winding insulator material.

Nuclear radiation induces lattice displacements in the copper stabilizer material. This dpa damage results in an increase in the resistivity of the copper and, therefore, in the amount of resistive heat generation, should a portion of the winding become normal. To ensure that the winding remains cryostable, the combined heat load from resistive heating, nuclear heating, and eddy current heating must be less than the critical neat flux for the selected conductor. The resistivity increase due to dpa damage and the volumetric heat generation rate due to nuclear heating are principal factors in determining the shield thickness in the inboard centerpost region.

A variety of conductor/coolant concepts have been considered for use in TFCX. Each of these concepts can be accommodated in the alloted Tf coil space envelope and is capable of meeting the field-on-axis requirement. These conductor/coolant concepts are as follows: 
1. Superfluid Cooled NbTi Conductor. A NbTi conductor (three-level Rutherford cable) is cooled in a bath of superfluid helium ( $\left.\mathrm{He}_{I I}\right)$ at a temperature of $1.8 \mathrm{~K}$. Use of $\mathrm{He}$ II coolant exitends the peak field capability of NbTi conductors to about io $\mathrm{T}$, compared to about $8 \mathrm{~T}$ when $4.2 \mathrm{~K}$ liquid helium $\left(\mathrm{He}_{\mathrm{I}}\right)$ is used as the coolant.

2. Forced Flow Conductor in Support Plates. A NbTi conductor in a stainless steel condi-t is force-cooled by pressurized supercritical helium, which exits the winding at a temperature of about $3.1 \mathrm{~K}$. The conduits are supported in grooved support plates similar to the Westinghouse Large Coil Program (LCP) coil.

3. Forced-Flow Conductor in Epoxy-Bonded Winding Pack. The conductor is similar to the previous sase; however, instead of grooved support plates, the cable-in-conduit is cowound with a stainless steel support channel, and the entire winding is epoxy impregnated.

4. Internally/Externally Cooled Conductor in Epoxy-Bonded Winding Pack. $\mathrm{Nb}_{3} \mathrm{Sn}$ strands in a conduit are cooled by stagnant supercritical helium inside the conduit. Heat is transferred through the conduit wall to an external $4.2 \mathrm{~K}$ bath of helium in grooves cut into the interpancake insulation.

In the TFCX-H option, the peak field at the superconducting TF coil is $\sim 8.0 \mathrm{~T}$, which can be achieved using any of the existing LCP conductors. For the present design, the General Dynami's LCP conductor concept is being used for illustrative purposes.

The copper insert coils in TFCX-H are energized apart from the superconducting coils. The machine may be operated with or without the copper coils energized; when the copper coils are energized, they increase the field-on-axis by $\sim 1.3 \mathrm{~T}$ and account for $27 \%$ of the field-on-axis. The copper coils are operated as pulsed devices in order to avoid the excessive resistive power consumption that would result from steadystate energization.

Since copper has about the same shielding capability as steel, use of copper insert coils embedded in the shield offers the advantage of a more compact machine for a given ield-on-axis, since the copper serves a dual role. In addition, since the maximum field at the superconducting 
coils is less in the hybrid concept than in the al1-superconducting concept, the superconducting coils may be designed to a higher current density, helping to make the device more compact. On the other hand, the magnetic forces acting on the copper coils introduce additional complexity from the standpoint of structural support. Since the shield modules are physically separated from the superconducting coils, the centering force developed on the copper coils cannot be transmitted to the bucking post and must be reacted in wedging action. In addition to requiring precise fit-up of the shield modules, this purtion of the shield must be fabricated from a high-strength steel in order to avoid an excessive radial build. The out-of-plane (overturning) forces on the copper insert coils also present a structural support problem because of the limited space available inside the bore of the superconducting coils to accommodate intercoil support structure (ISS) for the copper insert coils.

Table 7.3 lists the TF magnet system parameters for the all-superconducting and hybrid options.

During normal operation, there are distributed forces acting along the periphery of the coil, both in and normal to the plane of the coil. The in-plane forces are radially inward and are reacted principally by the bucking post. The out-of-plane forces on the TF coil result in a large overturning moment that tends to tip the TF coils about their horizontal axis of symetry. This overturning moment is reacted by the ISS, which joins adjacent TF coils at the top and bottom.

To permit removal of torus sectors, the re is an "open window" region between adjacent TF coils extending from the horizontal midplane of the TF coils. The upper and lower portions of the TF coils arn joined to the ISS modules. Support of the open window region of the TF coils against out-of-plane loads is one of the more difficult TF coil design problems. The need to maintain the open window rules out the use of shear panels or cross-bracing between TF coils in the midspan region. The problem is intc isified by the pulsed nature of the out-of-plane loads, which leads to a comparatively low design stress ievel because of flaw growth and fracture mechanics considerations. 
Table 7.3. TFCX TF magnet system parameters

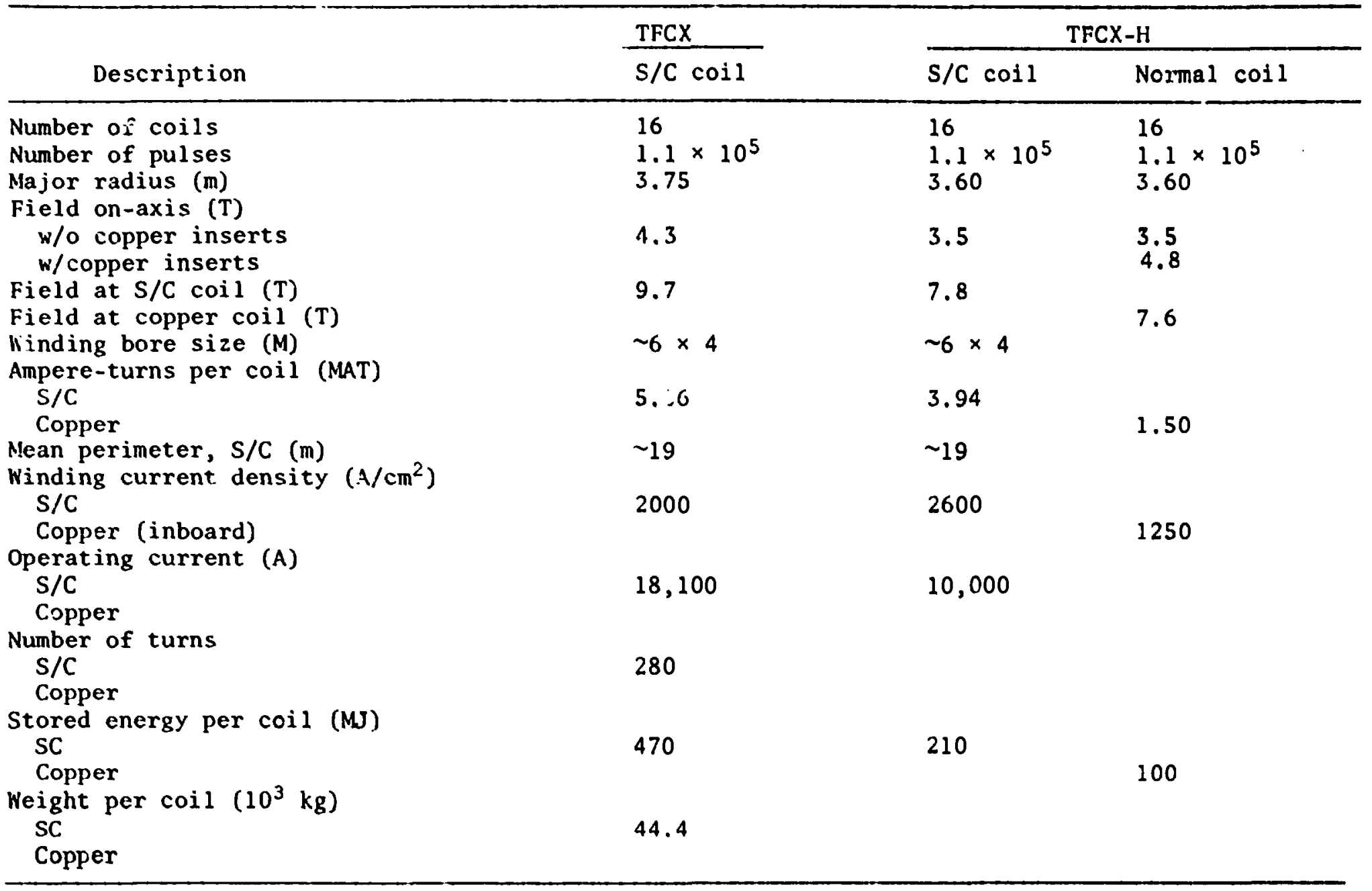


The TF coils are contained within a common vacuum vessel. The vacuum vessel is designed to enclose the open window region of each $T F$ coil in a manner resembling the fingers of a glove. In this way, the open access region between TF coils is preserved.

\subsection{PF COIL SYSTEM}

The PF coil system consists of $O H$ solenoid that provides energy to the plasma during the burn phase and a set of EF coils to maintain plasma equilibrium and produce a plasma triangularity of 0.3 . All PF coils are located outside the bore of the TF coils and are superconducting. A constraint adopied in determining the size and location of the PF coils is to limit the field in each of these coils to $88 \mathrm{~T}$ in order that NbTi conductors may be used.

The PF system performs two main functions: (1) to shape and equilibrate the plasma and (2) to inductively heat the plasma. The design objective is to satisfy these requirements at minimum PF system cost. Individual coil locations should be such that any coil could be easily maintained and replaced if necessary. The PF coil also must not inhibit maintenance or replacement of any other subsystem.

The functional requirements that must be satisfied by the PF coils are (1) the PF system must provide 0.3 triangularity with 1.6 elongation of the plasma, (2) the peak field at any coil must not exceed $8 \mathrm{~T}$, (3) adequate volt-seconds must be provided to allow for a 300-s burn, and (4) the PF configuration must be consistent with the TF and other structures.

The PF coils must be designed to be stable for operational heat loads. They must be structurally adequate to carry magnetic loads, dead weight, and $1-g$ seismic loads. The coils must be capable of being rapidly discharged from the fully charged state without damage and must survive all credible abnormal conditions without damage.

All of the PF coils are categorized as semi-permanent structures (i.e., they are to be designed for the life of the device and are not intended to be replaced or repaired). Nevertheless, the desigr must be 
such that coils can be replaced, if necessary. The design criteria for the PF coi: are the same as those for the IF coils discussed above.

Key features of the solenoid design are a double-pancake winding layout, modular design with modules powered by separate leads, a glass epoxy helium vessel to avoid induced currents, leads located in the central bore to minimize space required, and a solenoid supported off the TF ISS. The conductor for the solenoid is a 50-kA NbTi, copper-stabilized, pool-boiling, liquid-helium-cooled, flat cable. The conductor consists of 36 subcables around a central core made up of two strips of Nitronic 40. The use of two strips makes the core more flexible than a singlethickness strip would be, facilitates winding, and reduces eddy-current losses when an insulating material sheet is sandwiched between the two strips. Each subcable consists of six insulated - uperconducting strands around a stainless steel strand. The stainless steel strand increases the tensile strength of the subcable. The turn-to-turn insulation consists of G-10 spacers cowound with the conductor.

Key design features of these superconducting ring coils are (1) a 50-kA cable conductor; (2) pancake winding; (3) a stainless steel, ringstiffened case to provide structural support and to contain helium; (4) sliding pedestal supports to transmit dead weight, seismic loads, and the resultant out-of-plane magnetic load to the ISS; (5) a coil case designed to equilibrate magnetic bursting load and magnetic bending loads; (6) dielectric breaks in the case to reduce induced poloidal currents; and (7) joints sealed by welded omega seals or double 0-rings. The conductor being used for the superconducting rine coils is similar to that chosen for the solenoid, that is, a 50-kA bath-cooled (by liquid heliur. at atmospheric pressure), cabled NbTi conductor.

The arrangement of the Oll solenoid and the superconducting EF ring coils in the TFCX-S design is shown in Fig. 7.2.

\subsection{CRYOSTAT}

A common vacuum cryostat contains all of the superconducting coils. The cryostat has separate individual enclosures for the outboard legs of the TF coils. Thic approach maintajins the good access between the TF 


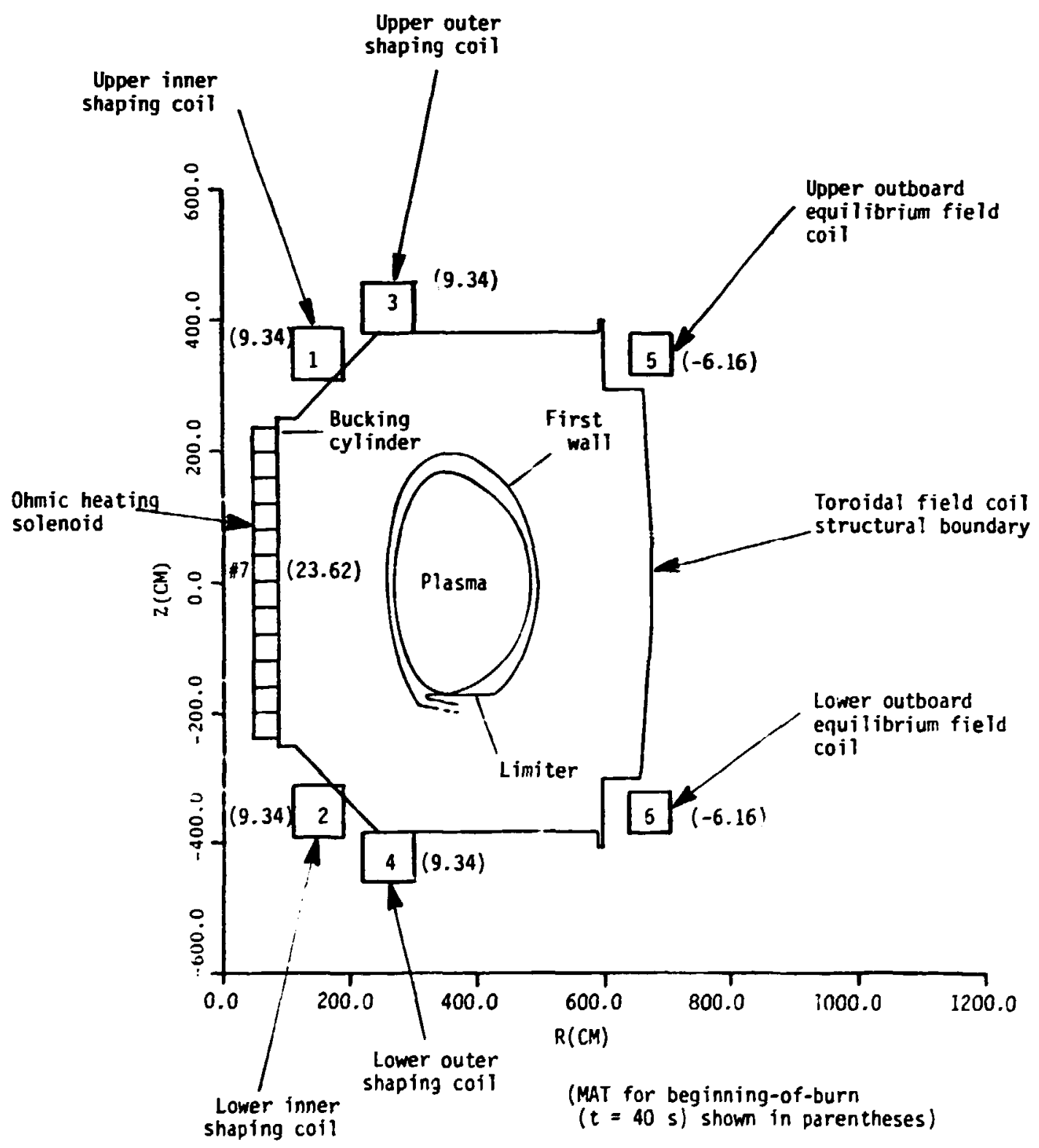

Fig. 7.2. Arrangement of $\mathrm{OH}$ solenoid and superconducting EF ring coils in the TFCX-S design. 
coils and requires no penetration of the cryostat boundary for torus access. This approach also separates the warm and cold components of the configuration. The key elements of the cryostat vessel include a cold wall inside the vacuum vessel following the contours of the vacuum vessel and individual heliun vessels for each superconducting coil.

The vacuum vessel is a shell-of-revolution around the entire magnetic system except for a portion of the vertical legs of the TF coils at their outer extremity, where openings are left to permit access to the torus. (Glovelike extensions of the vessel enclose these vertical legs.) The material is type 304 stainless steel. Preliminary calculations show that insulatirg breaks wi?l not be needed. 


\section{NUCLEAR SYSTEMS}

The nuclear systems consist of those components that make up the toroidal plasma chamber. For TFCX-S, those components include the torus support platform and pedestal, common vacuum buundary cryostat, removable shield module, pumped limiter, shield post, and first wall system. The general configuration of these components is shown in Fig. 8.1.

For TFCX-H those components include the torus support platform and pedestal, common vacuum boundary cryostat, removable shield module, copper TF coil/shield module, shield wedging post, connecting intercoil structure, fixed window shield modules, pumped limiter, and first wall system. Figure 8.2 shows the general arrangement of TFCX-H.

\subsection{TORUS AND SHIELD DESIGN}

Assembly of the shielding components within the common vacuum boundary cryostat on the torus support platform makes up the toroidal plasma chamber. The resulting torus is built within the bore of the 16 superconducting TF coils. The cryostat has window enclosures running from the toroidal chamber radially outward between the outboard TF coil legs. All the shielding components are installed through flanged openings in the 16 cryostat windows. This approach separates the hot and cold components and allows access to the plasma chamber without peretrating the separate cryostat vacuum vessel boundary for coils located internal to the reactor vessel.

The shielding components within the plasma chamber attenuate nuclear radiation, convert neutron kinetic energy into heat, provide for removal of this heat, and support the first wall and limiter components. Other system components such as $\mathrm{rf}$ heating, fueling, testing, diagnostics, instrumentation, and control are also installed within the shielding.

The inboard shield thickness for TFCX-S and TFCX-H is $0.58 \mathrm{~m}$ and $0.59 \mathrm{~m}$, respectively. The shielding materials are type 316 stainless steel $(80 \mathrm{v} / 0)$ and water $(20 \mathrm{v} / 0)$, with the innermost $0.3 \mathrm{~m}$ made of $\mathrm{B}_{4} \mathrm{C}$ 


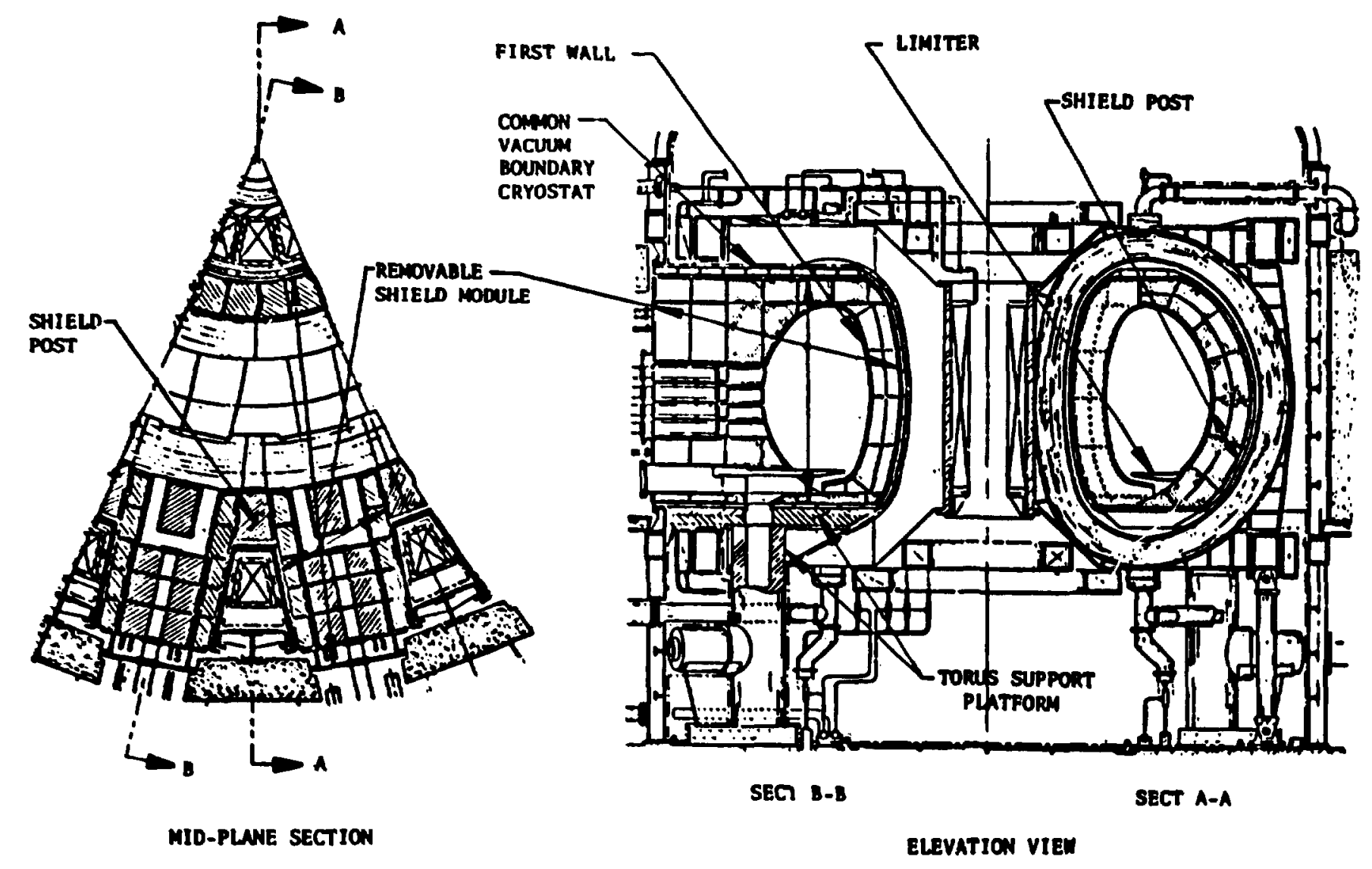

Fig. 8.1. TFCX-S general configuration. 

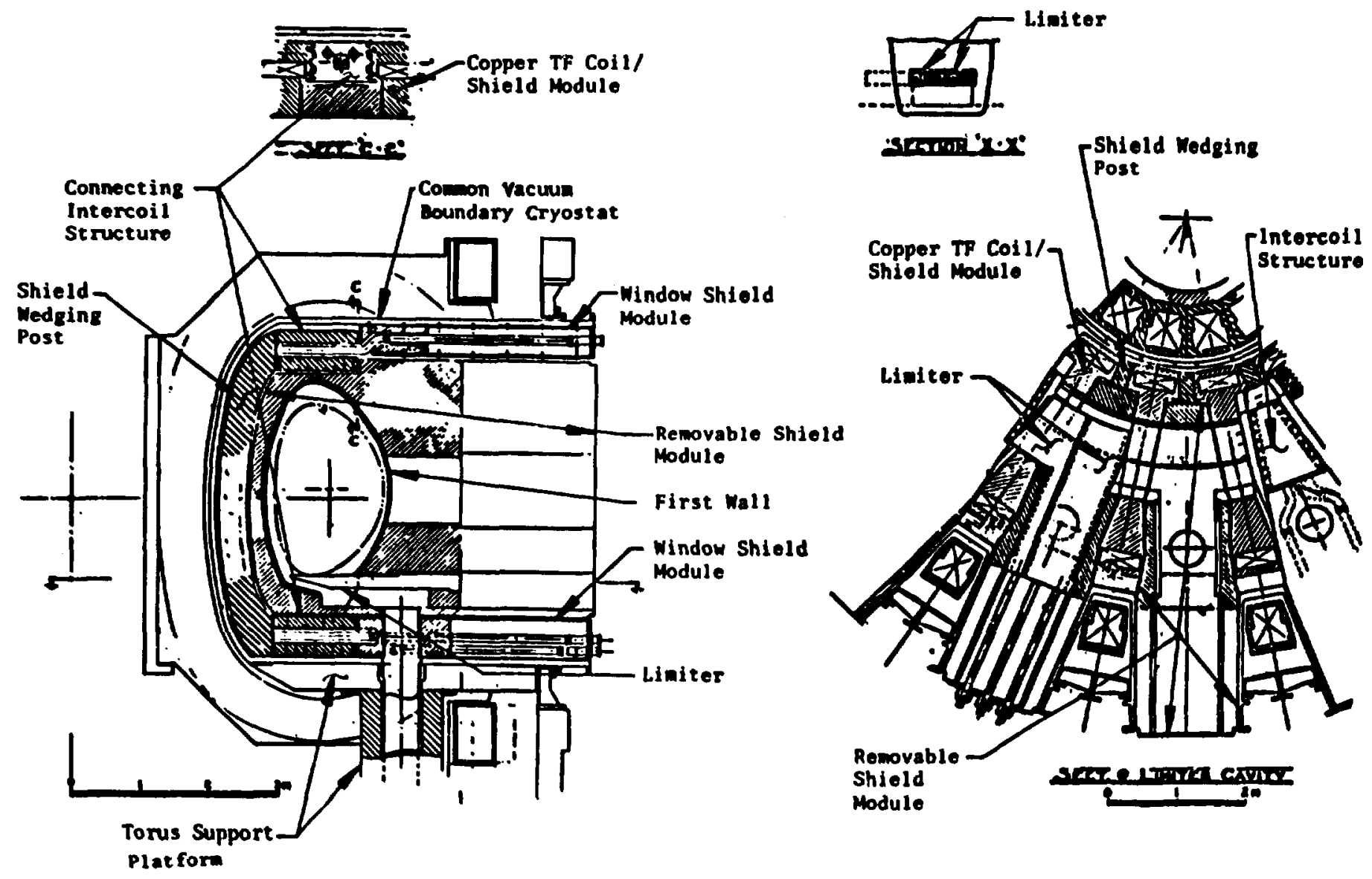

Fig. 8.2. TFCX-H general arrangement. 
contained in a thin wall container. The stainless steel is in plate form, with machined coolant passes, encased in a pressure-containing shell.

The outboard shield thickness for TFCX-S and TFCX-H is $2.2 \mathrm{~m}$ and $2.6 \mathrm{~m}$, respectively, in the window region. The shield materials are type 316 stainless steel $(60 \mathrm{v} / 0)$ and water $(40 \mathrm{v} / 0)$ for the first $0.70 \mathrm{~m}$ from the plasma. The stainless steel is in roughly spherical-shaped ball form $(1.27 \mathrm{~cm})$, procured from ball-bearing manufacturers. The remaining outboard shield material is water (95 v/o) contained in structural boxes. The shielding is contained in a compartmentalized stainless steel pressure vessel with layers of $\mathrm{B}_{4} \mathrm{C}(0.03 \mathrm{~m})$ and lead $(0.05 \mathrm{~m})$ on the outboard face, respectively.

The outboard shield thickness in the plane of the TF coil is $0.85 \mathrm{~m}$ for both the TFCX-S and TFCX-H configurations. The shield materials for TFCX-S are 316 stainless steel $(60 \mathrm{v} / 0)$ in the ball form and water $(40 \mathrm{v} / 0)$, with layers of $\mathrm{B}_{4} \mathrm{C}(0.03 \mathrm{~m})$ and lead $(0.05 \mathrm{~m})$ on the outboard face. The materials for TFCX-H will be the same, except that the percentages and form will be determined by structural requirements of the copper coil insert.

The shielding thicknesses were determined by the requirements to (1) limit the nuclear heating rate in the TF coil winding (inboard leg) to $1 \mathrm{~mW} / \mathrm{cm}^{3}$ and (2) 1 imit the shutdown dose rate after $24 \mathrm{~h}$ to $2.5 \mathrm{mrem} / \mathrm{h}$.

The structural component of the bulk shield is a welded structural box made of Nitronic 33. The bulk shield steel plates (Nitronic 33) in the inner shield region are bolted to each other and to the structural box and are coated with a dielectric $\left(\mathrm{Al}_{2} \mathrm{O}_{3}\right)$ to minimize eddy currents induced by plasma distuptions. The coolant water is channeled poloidally through machined grooves in the steel plates. There is no effort to seal the coolant pressure in the grooves. Rather, the box structure, stiffened by the bolted plates, carries the coolant pressures. The thickness of the plates and the spacing and size of the coolant grooves enable the desired distribution of steel and water to be achieved within $1 \%$. 
In the outer blanket region, the structure is a welded box that is used as a container for the stainless steel balls that fill the structural box in the first $0.70 \mathrm{~m}$ of the outer shield region.

The torus support system consists of 16 vertical columns, a toroidal support platform, and lateral support panels. The torus shield sectors are supported by the support platform and vertical columns, one of which is located under each shield sector. The lateral support panels provide a base for installation and removal of the shield sectors. All support system components are constructed of type 304 stainless steel and operate at room temperature. The vertical support column surround the pumping duct channels that pump out the plasma chamber and provide impurity control pumping during the burn.

Sector removal/replacement is accomplished by moving the sector radially outward on integrally mounted roller-dolly assemblies. Once clear of the device, the sector is transported by an overhead crane.

\subsection{FIRST WALL SYSTEM}

The interior of the plasma chamber is designed to protect the basic torus from heat loads and energetic particles generated by the plasma and to protect the plasma from deleterious impurities generated by interaction of the plasma with the interior surfaces. Of special concern are the inboard, top, and bottom surfaces that are subject to very high, but poorly defined, heat loads during disruption.

The TFCX first wall system includes all components which directly face the plasma. It consists of the pumped limiter on the bottom of the plasma cavity, passively cooled armor tiles on the inboard wall, and actively cooled sandwich panels on the outbuard wall. The panels are made of 316 stainless steel. The design concept is shown in Fig. 8-3. The pumped limiter is technically part of the first wall system, but due to its special function of impurity control, it is discussed separatcly.

The primary purpose of the first wall system is to accommodate the nominal heat loads during startup and burn and the anticipated disruption energy without replacement for the life of the device. The thickness 


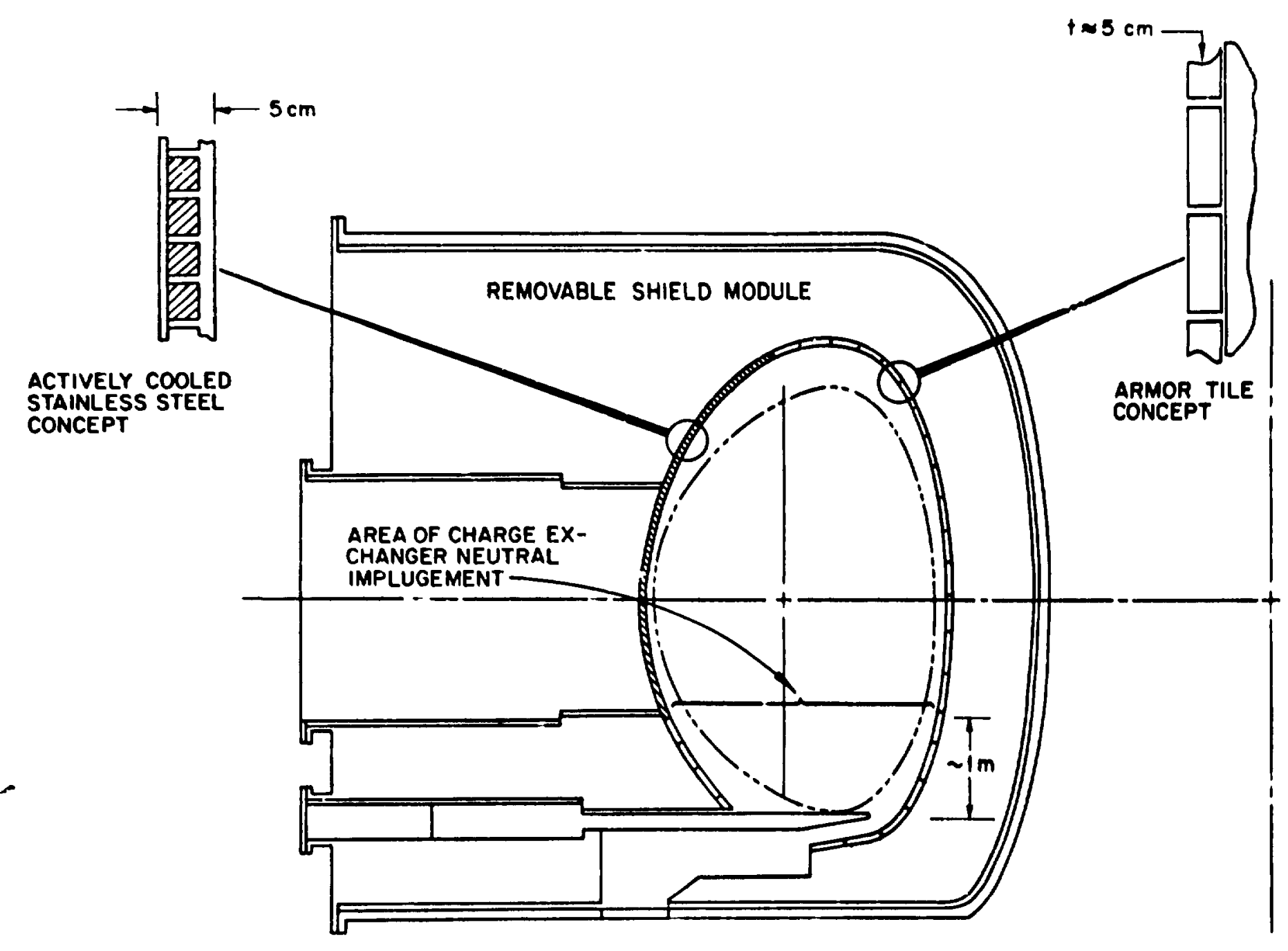

Fig. 8.3. TFCX first wall design concepts. 
for both the armor tiles and the stainless steel panels was assumed to be $5 \mathrm{~cm}$ in the radial build. Specific thermodynamic analyses using startup, burn, and anticipated disruption data for the TFCX must be performed in the future to confirm that $5-\mathrm{cm}$ thickness is the appropriate thickness. Candidate materials under consideration for the armor tiles presently include $\mathrm{BeO}, \mathrm{Be}, \mathrm{B}, \mathrm{B}_{4} \mathrm{C}, \mathrm{C}$ and possibly $\mathrm{SiC}$.

To accommodate the surface heat loads during normal operation, water-cooled first-wall panels are installed on the outboard shield facets. These panels are fabricated of type 316 stainless steel. The panels are a multipass design, with the coolant flowing toroidally back and forth through the panels in a serpentine pattern.

The panels are installed from the sides of the shield sectors. Removal of the sector is required in order to replace a panel. Panel replacement should be infrequent, however, since the panel life is expected to exceed the life of the device.

\subsection{IMPURITY CONTROL SYSTEM}

The TFCX employs a mechanical pumped limiter for particle and impurity control. The purpose of the limiter is to (1) establish the plasma edge, (2) provide a mechanism to remove helium ash and hydrogen particles from the plasma, and (3) help protect first-wall components from large particle and energy fluxes.

The limiter is located in the bottom of the plasma chamber mounted in a cantilever position from the outboard shield, as shown in Fig. 8.4. The limiter is divided into 32 segments, with two segments installed in each removable shield module through an access penetration. A flanged vacuum seal on each penetration at the outboard face of the shield module allows removal and installation of limiter segments without removing the shield module. The limiter segments come together in a radial, spokelike fashion forming a toroidally continuous limiter surface inside the plasma chamber, as shown in Fig. 8.5. The inboard tip of the limiter surface is $32 \mathrm{~cm}$ from the plasma tangent 1 ine. 


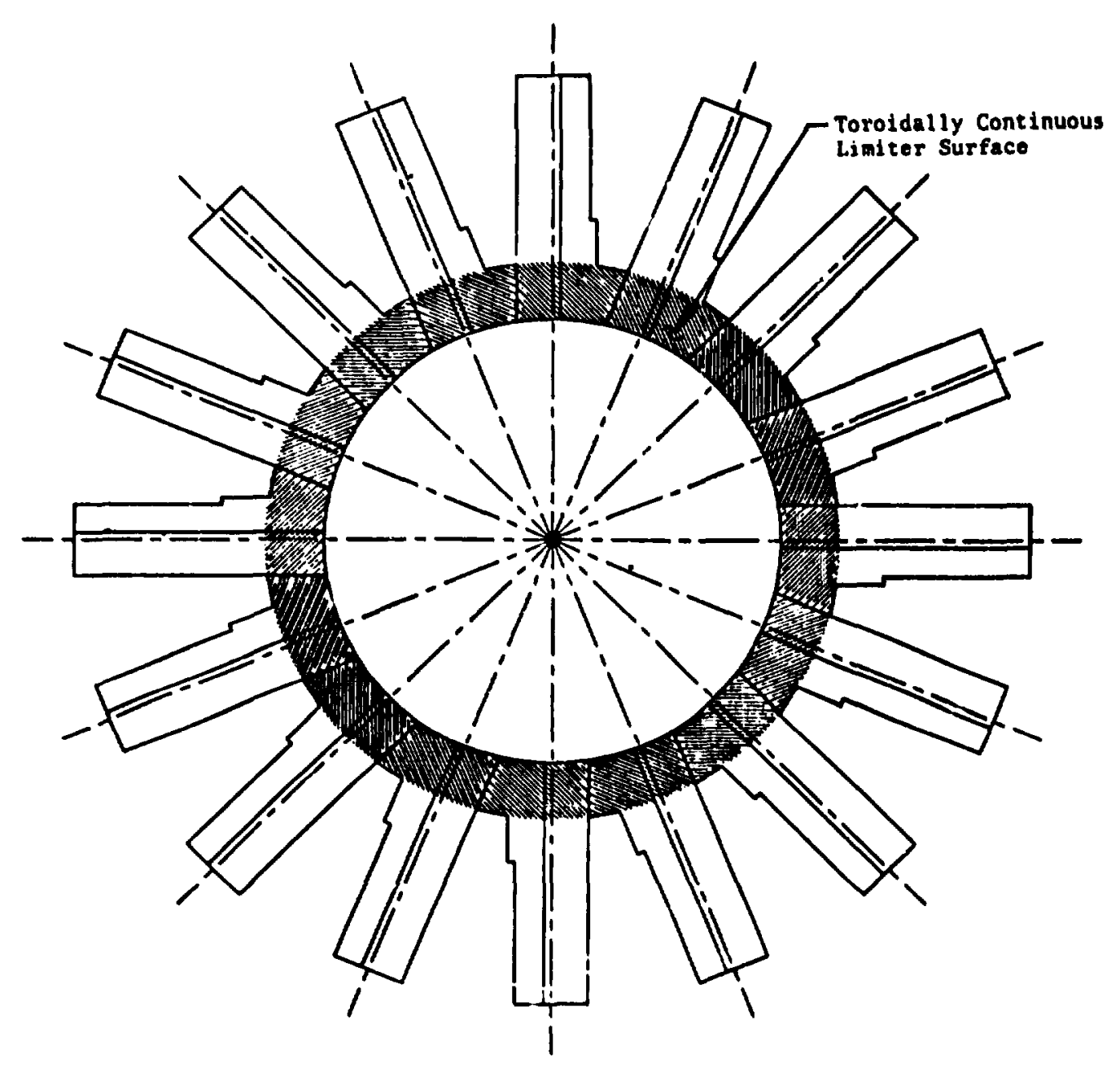

Fig. 3.4. Plan view of installed limiter segments. 


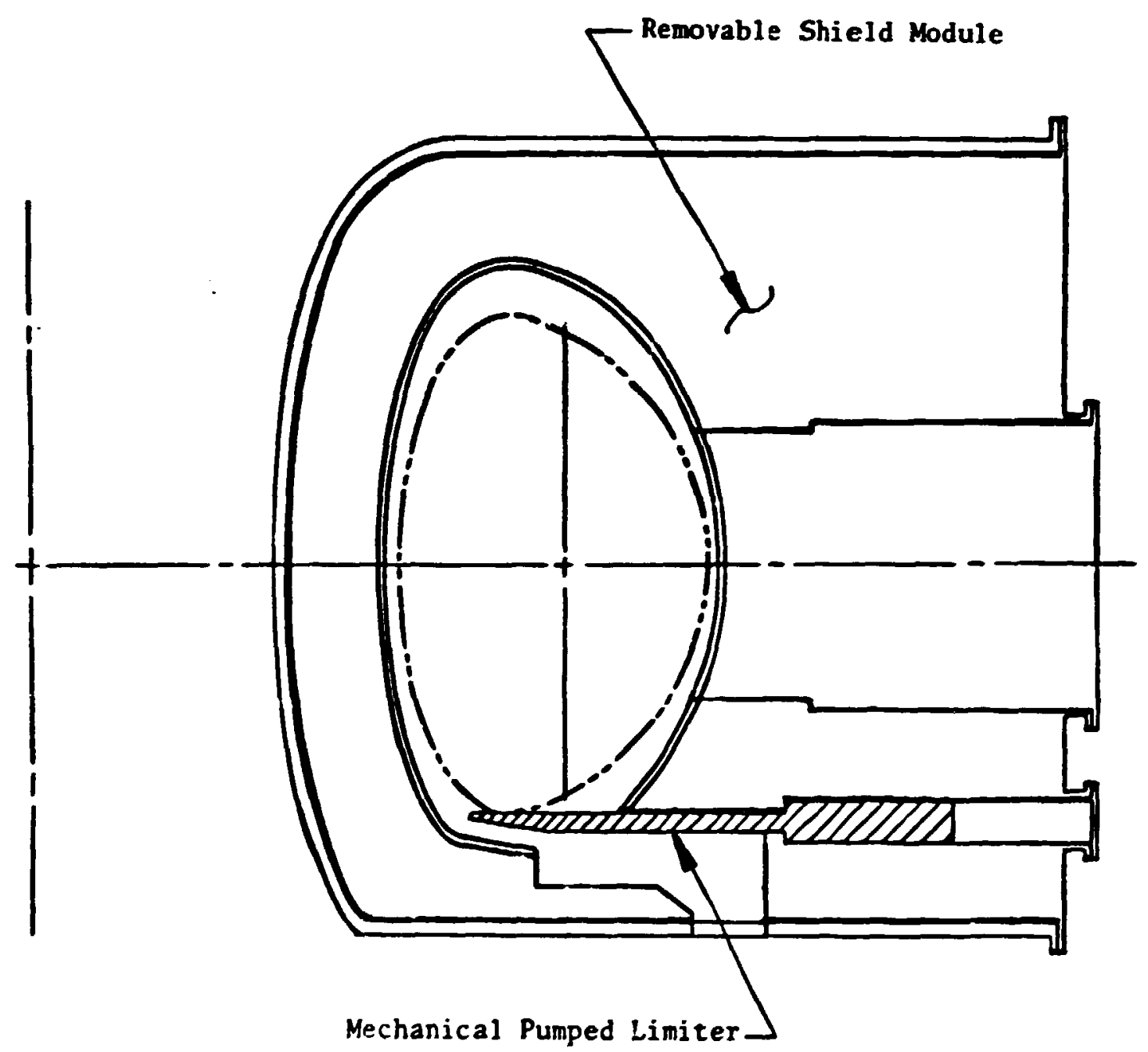

Fig. 3-5. TFCX limiter location. 
Each limiter segment has a reusable core structure and a replaceable heat ablative protective sleeve. The core structure is made of Nitronic 33 stainless steel, and the protective sleeve consists of a sandwich panel, copper substrate with brazed armor tiles. Candidate armor tile materials are as previously indicated. Both the core structure and the copper substrate are actively cooled. Limiter segments are electrically connected by pressurized bellows that activate copper contacts along one edge of each segment. 


\section{PLASMA HEATING SYSTEMS}

The plasma heating systems consist of those systems needed to perform plasma initiation, current ramp-up, and plasma bulk heating. The initiation phase includes the ionization or breakdown of the gas and heating beyond the point where radiation losses dominate the energy balance. During the current ramp-up phase, rf energy is planned to increase the current to near the final values. In the final phase, auxiliary heating provides the energy to reach and maintain the plasma burn conditions.

\subsection{PLASMA INITIATION AND STARTUP}

TFCX uses an rf system to provide initial heating. This system overcomes the need for a high-voltage plasma initiation and reduces the volt-seconds required from the PF system. Abcut $1 \mathrm{MW}$ of ECRH is applied when the gas is puffed into the tcrus. T:is $r f$ energy is sufficient to ionize and heat the electrons in the absence of a toroidal plasma current. The electron cyclotron wave is launched from the high-field side (HFS) of the torus using the extraordinary mode of propagation. Ionization occurs in a relativaly small vertical band around the major radius, whose radial position is determined by the frequency of the rf system and the magnitude of the toroidal field. A few hundred milliseconds after the ECRH is applied, the temperature at this vertical band of plasma exceeds the temperature at which radiation loss dominates. Plasma current is started after the electron temperature reaches $\sim 100 \mathrm{eV}$.

The ECRH system configuration consists of a high-power gyrotron oscillator that supplies a mode-tranducing launcher through an overmoded circular waveguide. The ECRH system is a single-frequency system (105 $\mathrm{GHz}$ ) that provides proneating of the electrons to a temperature of -50 to $100 \mathrm{eV}$, which is above the radiation harrier. The requirements of the ECRH system are given in Table 9.1 for both the TFCX-S and TFCX-H concepts. 
Table 9.1. ECRH preheating requirements for TFCX-S and TFCX-H

\begin{tabular}{lll}
\hline & TFCX-S & TFCX-H \\
\hline Type & ECRH & ECRH \\
Species & D & D \\
Pulse length capability (s) & 3 & 3 \\
Power (MW) & 1 & 1 \\
Frequency (GHz) & 105 & 115 \\
Field at startup (T) & 3.75 & 4.11 \\
Field on-axis (T) & 4.34 & 4.84 \\
Mode & $x^{a}$ & $x^{a}$ \\
Launcher position & $h^{a}$ & $\mathrm{HFS}$ \\
Injection angles (degrees) & 85 & 85 \\
\hline
\end{tabular}

$a_{X}$ - the extraordinary mode.

$b_{\text {HFS - the high-field side. }}$ 


\subsection{HEATING AND CURRENT DRIVE}

It is yroposed to use $\mathrm{rf}$ systems to ramp up the current subsequent to the iorization of the plasma. It is planned to ramp up the current slowly ( $\sim 30 \mathrm{~s})$ to its full value using about $13 \mathrm{MW}$ of LHRH power provided through Brambilla grills. This should permit driving the current up to about 7 MA. Table 9.2 sumarizes the requirements for current drive for both TFCX-S and TFCX-H.

The bulk heating of the plasma is planned to occur following the current ramp-up. During this period, the bulk heating is provided by about $25 \mathrm{MW}$ of ICRH from a poloidal stack of four ridge-loaded waveguides. The ICRH system has come tuning capability to allow operation at lower magnetic fields and/or with different fuel species during the check out phase. This system will raise the plasma temperature and density to operating conditions in about $10 \mathrm{~s}$. The requirements for the ICRH bulk heating system are given in Table 9.3 for TFCX-S and TFCX-H.

During the burn portion of the plasma cycle, current is supplied by the $\mathrm{OH}$ transformer. If operation is condurted in nonignited conditions (i.e., the device is operated in a driven mode), bulk heating of the plasma is provided by the LHRH and ICRH systems. Burn pulse lengths of $\sim 300$ s have been specified for both TFCX-S and TFCX-H.

The requirements for the plasma heating systems are traceable to plasma performance through scaling laws that are based on analyses/ modeling and experimental results. At present, the understanding of current drive ani heating efficiency of various modes is growing rapidly. Because of this, the requirements are continuously evolving. If a single system could be used to perform all functions at the required performance level, this would be ideal; however, at present there is little or no substantiation that such a concept will evolve from presentday experiments. Startup experiments with $r f$ are under way at PPPL using LHRH. If successful, consideration could be given to eliminating the ECRH preheating system. Current-drive experiments using ICRH at PPPL and bulk heating experiments vising LHRH at PPPL and Massachusetts Institute of Technology (MIT) are either planned or under way. The results of these could allow elimination of one system and lower the 
Table 9.2. Current-drive requirements for TFCX-S and TFCX-H

\begin{tabular}{|c|c|c|}
\hline & TFCX-S & TFCX-H \\
\hline $\begin{array}{l}\text { Type } \\
\text { Initiation and startup } \\
\text { Bulk heating } \\
\text { Burn }\end{array}$ & $\begin{array}{l}\text { LHRH } \\
\text { LHRH } \\
\text { OH }\end{array}$ & $\begin{array}{l}\text { LHRH } \\
\text { LHRH } \\
\text { OH }\end{array}$ \\
\hline Species & $H, D, D-T$ & $H, D, D-T$ \\
\hline $\begin{array}{l}\text { Pulse length (s) } \\
\text { H or D } \\
\text { DT }\end{array}$ & $\begin{array}{l}340 \\
40\end{array}$ & $\begin{array}{l}340 \\
40\end{array}$ \\
\hline $\begin{array}{l}\text { Power (MW) } \\
\text { H or D } \\
\text { D-T } \\
\text { Control }\end{array}$ & $\begin{array}{l}14 \\
14 \\
10: 1\end{array}$ & $\begin{array}{l}12 \\
12 \\
10: 1\end{array}$ \\
\hline Frequency (GHz) & 2.73 & 2.73 \\
\hline Field on axis & 4.34 & 4.84 \\
\hline $\begin{array}{l}\text { Density }\left(\mathrm{cm}^{-3}\right) \\
\text { High } \\
\text { Low }\end{array}$ & $\begin{array}{l}1.8 \times 10^{14} \\
1.3 \times 10^{13}\end{array}$ & $\begin{array}{l}1.8 \times 10^{14} \\
1.3 \times 10^{13}\end{array}$ \\
\hline Mode & 0 & 0 \\
\hline Launcher position & $\begin{array}{l}\text { Midplane } \\
\text { LFS }\end{array}$ & $\begin{array}{l}\text { Midplane } \\
\text { LFS }^{\circ}\end{array}$ \\
\hline $\begin{array}{l}\text { Spectrum control }\left(n_{11}\right) \\
\text { Low density } \\
\text { High density }\end{array}$ & $\begin{array}{l}1.4 \pm 0.1 \\
2.1 \pm 0.1\end{array}$ & $\begin{array}{l}1.4 \pm 0.1 \\
2.1 \pm 0.1\end{array}$ \\
\hline
\end{tabular}


Table 9.3. Bulk heating requirements

\begin{tabular}{|c|c|c|}
\hline & TFCX-S & TFCX-H \\
\hline Type & ICRH & ICRH \\
\hline $\begin{array}{l}\text { Species } \\
\text { Startup } \\
\text { Bulk heating } \\
\text { Burn }\end{array}$ & $\begin{array}{l}\mathrm{H}, \text { DEH } \\
\text { minority } \\
\mathrm{H}, \mathrm{D}, \mathrm{D}-\mathrm{T} \\
\mathrm{H}, \mathrm{D}\end{array}$ & $\begin{array}{l}\text { H, DEH } \\
\text { minority } \\
\text { H, D, D-T } \\
\text { H, D }\end{array}$ \\
\hline $\begin{array}{l}\text { Harmonic } \\
\text { H } \\
\text { D } \\
\text { D-T }\end{array}$ & $\begin{array}{l}\text { lst } \\
\text { 2nd } \\
\text { 2nd } D^{+}\end{array}$ & $\begin{array}{l}\text { 1st } \\
\text { 2nd } \\
\text { 2nd } D^{+}\end{array}$ \\
\hline Frequency $(\mathrm{GHz})$ & 0.0651 & 0.0726 \\
\hline Field on-axis $(\mathrm{T})$ & 4.34 & 4.84 \\
\hline Mode & $x^{a}$ & $x^{a}$ \\
\hline Launcher Position & $\begin{array}{l}\text { Midpl ane } \\
\text { LFS } b\end{array}$ & $\begin{array}{l}\text { Midplane } \\
\text { LFS }^{b}\end{array}$ \\
\hline $\begin{array}{c}\text { Power (MW) } \\
\text { H, D } \\
\text { D-T } \\
\text { Control }\end{array}$ & $\begin{array}{l}36 \\
23 \\
20: 1\end{array}$ & $\begin{array}{l}42 \\
25 \\
20: 1\end{array}$ \\
\hline $\begin{array}{l}\text { Pulse length (s) } \\
\text { H, D } \\
\text { D-T }\end{array}$ & $\begin{array}{l}340 \\
40\end{array}$ & $\begin{array}{l}340 \\
40\end{array}$ \\
\hline
\end{tabular}


cost and complexity considerably. The use of the three rf systems combined with the OH transformer provides a very flexible system and allows a wide variety of experiments and scenarios to be considered, as were briefly described in Sect. 5 .

Figure 9.1, 9.2, and 9.3 illustrate the basic configurations and features currently envisioned for the ECRH, LHRH, and ICRH systems, respectively. 


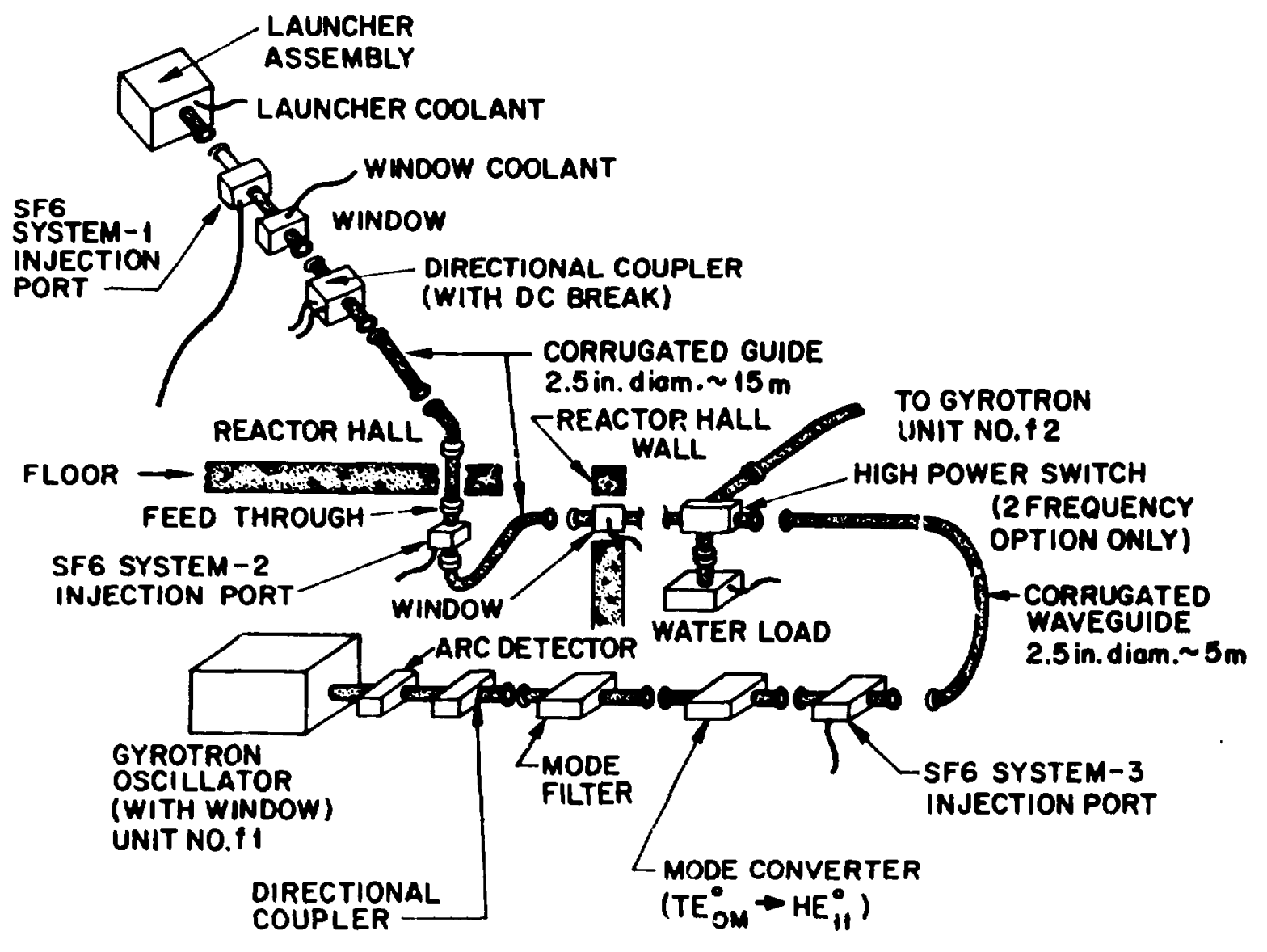




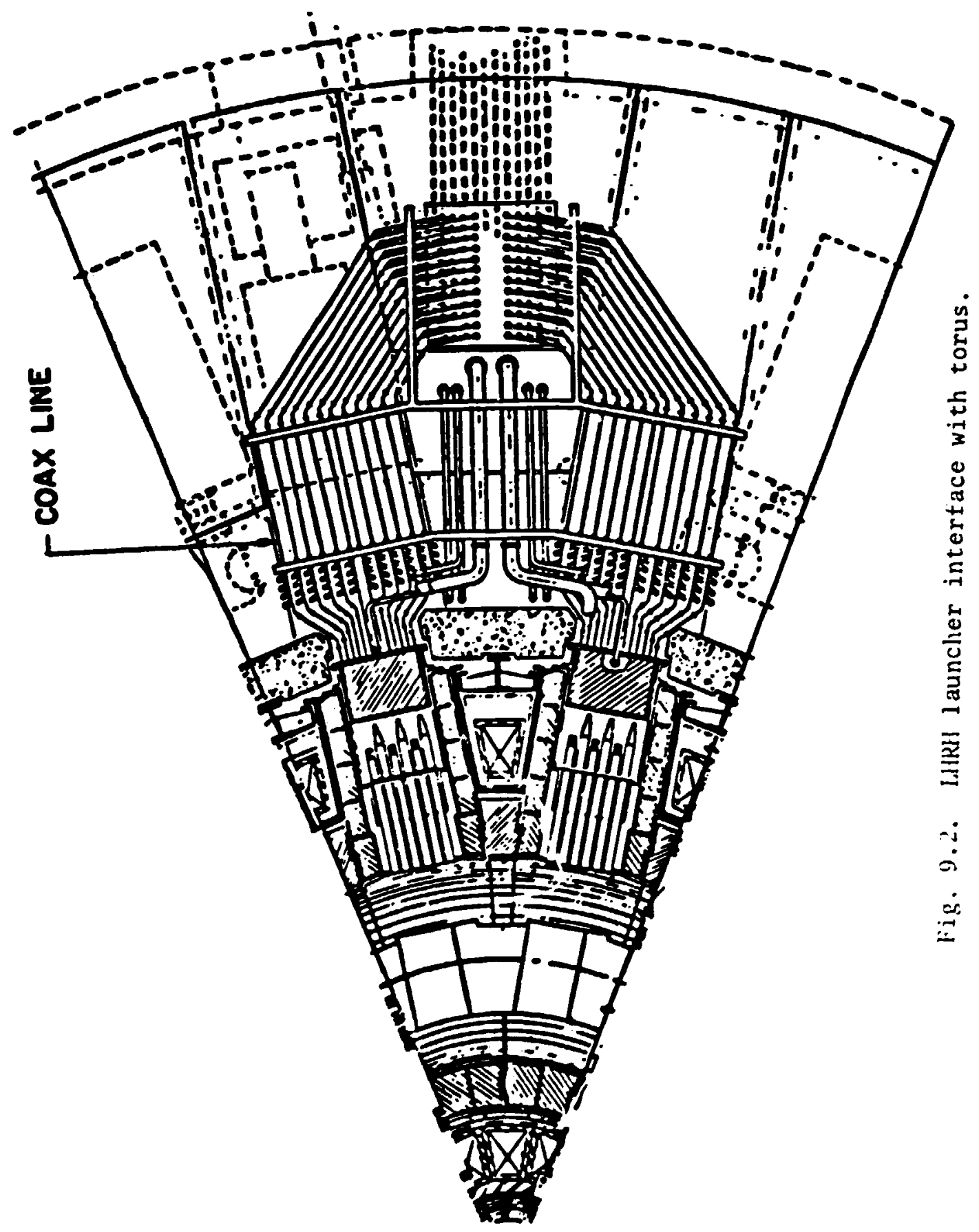




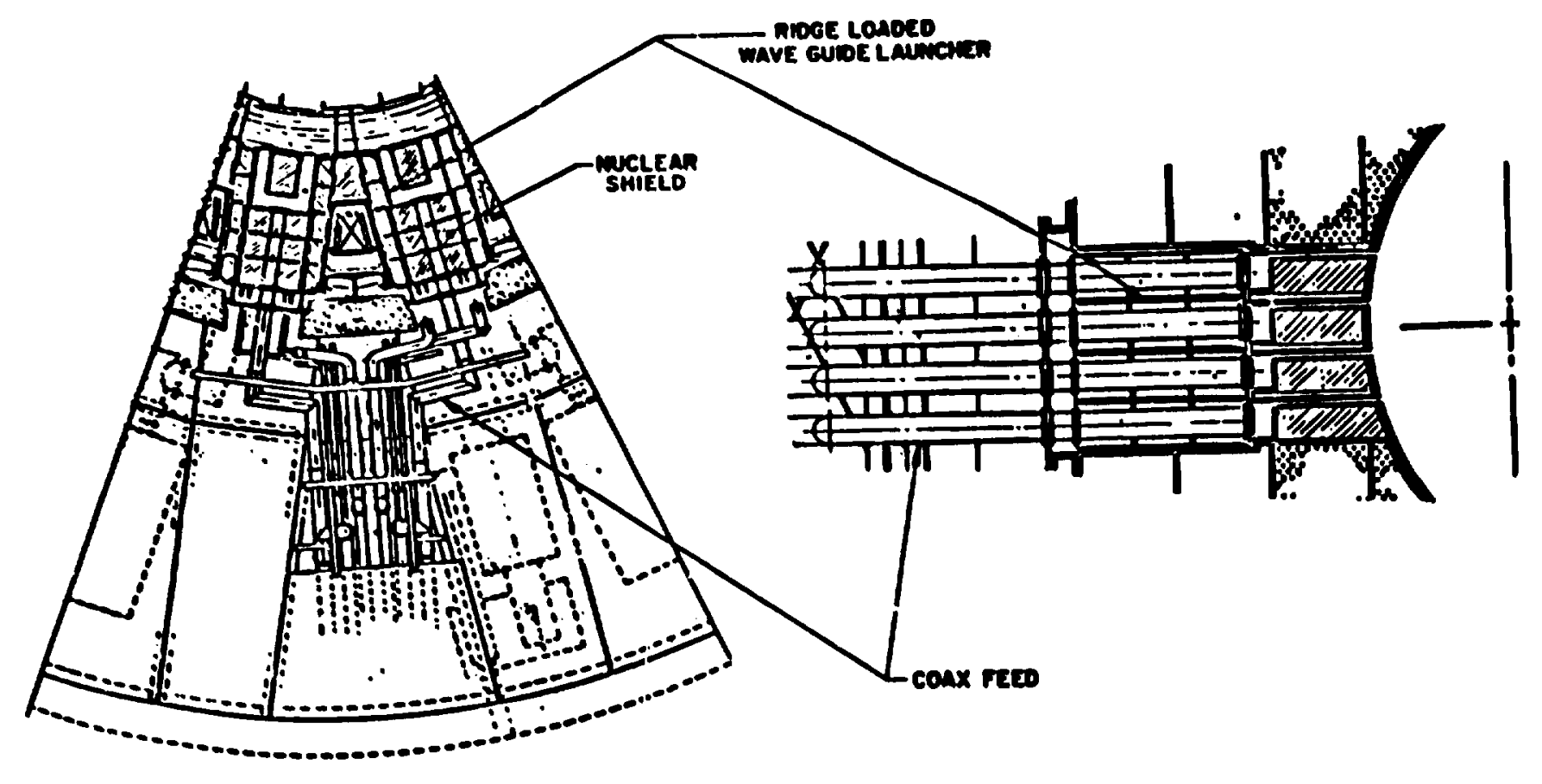

Fig. 9.3. ICRH launcher interface. 


\subsection{SHUTDOWN}

During shutdown, the fusion burn is quenched before the current is quenched. Under normal conditions, the fusion quench is initiated by termination of fueling while maintaining or possibly enhancing plasma exhaust through the pump limiter. Transpor estimates have suggested a time scale of $\sim 2 \mathrm{~s}$ is required to return to the ohmic state. A plasma density of $\sim 2 \times 10^{13} \mathrm{~cm}^{-3}$ is assumed at the end of the fusion quench. Conceptually, the plasma current shutdown is the reversal of the plasma current ramp-up. As the plasma radius decreases, the value of $q_{\Psi}$ at the plasma edge should be maintained or increased as much as possible during this phase to avoid disruptive termination of large plasma current.

\subsection{PUMPDOWN}

The neutral density in the chamber at the end of the current quench is expected to be $\sim 3 \times 10^{-4}$ torr. It is assumed that the residual gas is relatively clean, with only negligible amounts of helium and low- $z$ impurities. Pumpdown to $3 \times 10^{-6}$ torr in $630 \mathrm{~s}$ is assumed during this phase. 


\section{ELECTRICAL SYSTEMS}

The TFCX electrical systems consist of power handling and conve:sion; energy storage; and plasma diagnostics, instrumentation, data acquisition, and control.

\subsection{POWER HANDLIN'; AND CONVERSION}

The power handling and conversion system consists of the ac p. er system and the power supplies and protection system, for the TF and PF coils. The ac power includes the $60-\mathrm{Hz}$ input from the utility company, the associated substations, and an intercomplex distribution system.

Since there has been no site selection at this time, the specific utility line has not been included in the studics. It is understood that there may be some load leveling required, but operation in general will not be curtailed by the load requirements for the superconducting coil options. Two power lines will be required for sites that are not located near major power lines or generating capacity. One line will provide the pulsed power and the other steady-state power and general facilities. In addition, In addition, standby diesel generators and uninterruptible power supplies are needed for backup power for essential loads. The major ac power loads for TFCX-S and TFCX-H are given in Table 10.1.

The steady-state or longer duration loads are listed in the upper part of the table. Pulsed loads that do not exist during the burn phase are listed in the lower part of the table. The pulsed loads listed in the lower part of the table are five to ten times higher than the continuous loads. The ECRH load is small compared to the others. Both LiRF and ICRH power loads exist for the entire 30-s startup. If these loads can be ramped on or applied in steps, they can be connected directly to the utility line. The large pulsed PF coil power supply load is ramped up on over a 30 -s period. Therefore, the ', F coil load can be connected directly to the utility substation. For the same reason, the TFCX-H resistive copper (RC) TF coil power supply load can be connected directly t's the uility subslation. 
Table 10.1. Major Ac power system loads

\begin{tabular}{|c|c|c|}
\hline Description & $\begin{array}{c}\text { TFCX-S } \\
\text { (MW) }\end{array}$ & $\begin{array}{c}\text { TFCX }-\mathrm{H} \\
\text { (MW) }\end{array}$ \\
\hline \multicolumn{3}{|l|}{ Steady power } \\
\hline Facility power & 15 & 15 \\
\hline Power to SC TF PS & 2.4 & 2.4 \\
\hline Power to RC TF PS & & 50.0 \\
\hline Power to PF coil burn PS & 2.4 & 2.3 \\
\hline Power to cryogen system & 18.9 & 9.2 \\
\hline Power to cooland system & 6.4 & 7.6 \\
\hline Power to fuel processing system & 5.1 & 5.6 \\
\hline Power to vacuum system & 2.7 & 2.1 \\
\hline Total & 53 & 94 \\
\hline \multicolumn{3}{|l|}{ Pulsed power } \\
\hline Power to LHRH RS $(40-\mathrm{s})(40$ to $340 \mathrm{~s}$ ) & 39 & 33 \\
\hline Power to ICRH PS (40-5) & 45 & 68 \\
\hline Power to ECRH (3-s) & 5 & 5 \\
\hline Power to PF coil pulsed PS & 190 & 220 \\
\hline Counterpulse PS & & 50 \\
\hline Power to RC TF coil PS & & 20 \\
\hline Total & 279 & 396 \\
\hline
\end{tabular}


Based on the above considerations and the number of electrical loads, a pulsed substation is required that can handle about a 480-MW peak load for TFCX-H and $325 \mathrm{MW}$ for TFCX-S. All the steady-state loads that do not require normal voltage regulation can also be connected to this substation. All other loads must be connected to the facility substation, which provides better voltage reguiation. Major essential power loads (listed in Table 10.2) require backup power for operation when the utility power fails. The atmospheric detritiation system is much larger than all other essential power loads. About $40 \mathrm{kw}$ of uninterruptible power is needed for safety-related instrumentation and control.

\subsection{ENERGY STORAGE}

The er.ergy storage for the superconducting options will be essentially zero. The long rise time and the long pulse essen ia?ly eliminate the advantage or need for on-site energy storage. There may possibly be a need for transient control power that world result in a few megajoules of energy storage, but this would be much less than now used in Doublet III or TFTR.

\subsection{PLASMA DIAGNOSTICS, INFORMATION, AND CONTROL SYSTEMS}

The diagnostic requirements will include the same diagnostics as on present operating tokamaks, but there will also be the need for advancements in features silch as current density measurements and plasma shape. The neutron and radiation environment will be greatly increased over present devices, as is generally recognized. This will require extensive remote handling and/or shielding. In addition, the longpulse periods will require development of steady-state data output rather than one data point per pulse. The instrumentation for measuring the plasma loop current for a $300-s$ stcady-state flattop will require redevelupment of the existing technology. 
Table 10.2. Essential power requirements

\begin{tabular}{lc}
\hline \multicolumn{1}{c}{ Load description } & Power (kw) \\
\hline Ezergency tritium cleanup & 3000 \\
Fuel reprocessing & 500 \\
Escential HVAC & $20 C$ \\
Uninterruptible power & 20 \\
Data system & 50 \\
Instrumentation and control & 50 \\
Essential lights & 50 \\
\hline
\end{tabular}




\section{AUXILIARY SYSTEMS}

The auxiliary systems are defined as those supporting systems that, though not physically part of the device, are closely integrated with it in terms of systems operation. The fueling system, vacium pumping system, tritium systems, and remote maintenance equipment comprise the auxiliary systems and are briefly described below.

\subsection{FUELING SYSTEM}

The fueling system consists of gas puffers and pellet injectors. Prios to startup, the fuel gas puffers are used to fill the torus with deuterium to the desired pressure. Gas putfing continues until a plasma of $\sim 1 \mathrm{keV}$ is established. Frozen deuterium and tritium pellets are then injected to control the plasma density.

After the startup is completed, fuel pellets are injected to control che average plasma density and the gas injectors may be used to contro] the background gas pressure.

During early phases of operation, when the hydrogen n's.sma density and temperature are an order of magnitude lower than design conditions, the gas injectors may provide for all the fueling needs. Fueling in later phases of operation with higher plasma density and temperature requires frozen pellet injection for deeper penetration into the plasma. To accommodate all phases of operation, the in: ectors should have the capability of injectiry all three hydrogen isotopes: protium, deuterium, and tritium. Different pellet formers may have to be substituted when changing from hydrogen to deuterium-tritium operation and vice versa. Alternatively, three independent pellet formers may be employed.

Although large pellets are desirable for deeper penetration into a dense plasma, the pellet mass is 1 imited to about $10 \%$ of the plasma mass to prevent large perturbations in plasma density and temperature. This consideration and the particle confinement time determines the pellet size and pellet rate at the design point. The pellet size is about $3 \mathrm{~mm}$ and the burn pellet rate is about $6 \mathrm{~s}^{-1}$, which needs to be doubled 
to allow for uncertainties in confinement time and the additional acceleration needs during startup. The pellet injector and gas injector $x$ equirements data are summarized in Tables 11.1 and 11.2 , respectively. The pellet velocity is limited to about $2 \mathrm{~km} \cdot \mathrm{s}^{-1}$ based on target goals of the pellet injector development program. A higher velocity would be desirable when operating near design point conaitions. At these conditions, a 3-mm-dia pellet injected at $2 \mathrm{~km} \cdot \mathrm{s}^{-1}$ will penetrate the plasma about $25 \%$ of the minor radius.

To reduce potential tritium hazards, it is desirable to minimize the pellet injector tritium inventory. Based on a batch-type pellet former, the tritium inventory necessary for a 300-s burn is about $6 \mathrm{~g}$. If repeated 300-s burn cycles are desired, the inventory would be about $10 \mathrm{~g}$, to allow for freezing one batch while injecting the otiser. Augertype pellet former-injectors could conceivably reduce the inventory to less than $6 \mathrm{~g}$ for continuous operation.

The most effective location for injecting gas is on the underside of the plasma in the vicinity of the limiter blade. The cost of gas injectors is more than an order of magnitude lower than pellet injectors. For this reason, two independent gas injector systems can be justified, each having the capability of injecting at eight locations. Gas injection mass flow rate should be at least ten times the pellet injection rate to accommodate the uncertainty in gas recycle physics of the plasma and to provide for adequate response time in backfilling the torus and controlling the background pressure. The gas inistor system should have the capability of injecting any of the hydrogen isotopes or mixtures thereof. The gas flow may be pulse modulated or employ continuous modulation.

\subsection{VACUUM PUMPING}

The vacuum pumping system for TFCX is designed to pump down the torus vacuum vessel initįlly and between burns and to remove the gas load from the pumped limiter during the burn period. A detailed study of impurity removal during the burn, which accounts for the inceraction of the plasma and neutral gas particles, remains to be done. 
Table 11.1. Pellet injector requirements for TFCX

\begin{tabular}{|c|c|}
\hline Parameter & TFCX-S and TFCX-H \\
\hline $\begin{array}{l}\text { Number of dual-channel pellet } \\
\text { injectors }\end{array}$ & 1 \\
\hline $\begin{array}{l}\text { Pellet size (mg) } \\
\text { iritium } \\
\text { Deuterium }\end{array}$ & $\begin{array}{l}6.5 \\
4.3\end{array}$ \\
\hline Pellet diameter $(\mathrm{mm})$ & 3 \\
\hline $\begin{array}{l}\text { Pellet composition } \\
\text { Hydrogen phase } \\
\text { D-T vhase } \\
\text { Tritium pellet } \\
\text { Deuterium pellet } \\
\text { Mixed pellet }\end{array}$ & $\begin{array}{l}>99 \% \mathrm{H} 2 \\
>99 \% \mathrm{~T} 2 \\
>90 \% \mathrm{~T} 2 \\
\text { Recovery mixture }\end{array}$ \\
\hline Injector distance to plasma (m) & 5 \\
\hline $\begin{array}{l}\text { Magnetic field in vicinity of } \\
\text { pellet injector (T) }\end{array}$ & $\mathrm{TBD}$ \\
\hline Pellet velocity, $\max \left(m \cdot s^{1}\right)$ & $\geqslant 2000$ \\
\hline Pellet rate/channel, $\max \left(\mathrm{s}^{-1}\right)$ & 6 \\
\hline Maximum injection period (s) & 350 \\
\hline Low tritium leakage & (Double confinement) \\
\hline Low tritium inventory $(\mathrm{g})$ & 210 \\
\hline Availability $/ 8 \mathrm{~h}$ & $>0.95$ \\
\hline
\end{tabular}




$$
11-4
$$

Table 11.2. Gas injector requirements

\begin{tabular}{ll}
\hline \multicolumn{1}{c}{ Parameter } & \multicolumn{1}{c}{ TFCX-S and TFCX-H } \\
$\begin{array}{l}\text { Number of deuterium gas } \\
\text { injection systems }\end{array}$ & 2 \\
$\begin{array}{l}\text { Number of gas injection } \\
\text { nozzles }\end{array}$ & 8 \\
Maximum gas flow rate $\left(g \cdot \mathrm{s}^{-1}\right)$ & 0.3 \\
$\begin{array}{l}\text { Maximum valve pulse length }(\mathrm{ms}) \\
\text { Maximum valve pulse rate }\left(\mathrm{s}^{-1}\right)\end{array}$ & 150 \\
Gas composition & 5 \\
Availability/8 $\mathrm{h}$ & $\begin{array}{l}\mathrm{All} \text { hydrogen } \\
\text { isotopes and } \\
\text { mixtures thereof }\end{array}$ \\
\hline
\end{tabular}


Since the vacuum pumping requirements are not fully specified at this t1me, final selection of the high-vacuum pumps has not beer. made. The two prinary candidates are (1) turbomolecular pumps and (2) compound cryocondensing/cryosorption pumps. An alternative might be a combination of the two. The following description assumes the use of turbomolecular pumps.

Particles enter the toroidaly continuous, 0.1-m-high pumping slot underneath the limiter blade. The length of the slot is aboui $0.5 \mathrm{~m}$, corr.sponding to the position where plasma particles passing behind the limiter strike the backside of the limiter and are neutralized. The toroidally continuous slot is joined to 16 passages, one in each torus sector. Each of these passages is $0.3 \mathrm{~m} \mathrm{high,} 0.9 \mathrm{~m}$ wide, and $1.35 \mathrm{~m}$ long. An 0.5-m-diam vacuum duct iith two $90^{\circ}$ elbows connects each of these passages to a plate valve and $5 \mathrm{~m}^{3} \cdot \mathrm{s}^{-1}$ turbomolecular pump. There are eight valves and pumps arranged around the torus; therefor, iwo of the 0.5-diam ducts feed into each of the valve and pump assemblies.

The design guidelines assumed for the TFCX vacuum pumping system performance are listed in Table 11.3. An initial pumpdown pressure of less than $10^{-7}$ torr was set as a guideline to ensure that impurity levels will not affect plasma performance. The postshot pressure of $2.8 \times 10^{-3}$ torr was calculated by assuming that the neutral gas density in the plasma chamber after the burn is the same as the plasma density during the burn. The prefill or preshot pressure was assumed to be $10^{-5}$ torr, which ensures that the number of impurity molecules remaining in the chamber after pumpdown will be less than $1 \%$ of the number of $D-T$ full atoms. A pumpdown time of $30 \mathrm{~s}$ between consecutive burns was used.

The vacuum pumping system consists of 16 identical vacium ducts, one in each of the 16 torus sectors, as shown in Fig. 11.1. The 16 ducts merge into one below the limiter, diverging to pass between TF: coils on their way to the vacuum pumps. At the end of each pair of ducts is a turbomolecular pump, backed by a scroll pump.

The exhaust from all of the f.rst-stage scroll pumps is combined and passed to a second-stage scroll pump that compresses the gases to 
Table 11.3. Guidelines for TFCX vacuum pumping system purformance

\begin{tabular}{|c|c|c|}
\hline Condition & Guideline & Basis \\
\hline $\begin{array}{l}\text { Initial plasma chamber } \\
\text { pumpdown pressure (torr) }\end{array}$ & $<10^{-7}$ & $\begin{array}{l}\text { Maintain low impurity } \\
\text { level }\end{array}$ \\
\hline Postshot pressure (torr) & $2.8 \times 10^{-3}$ & Calculated \\
\hline Preshot pressure (torr) & $10^{-5}$ & $\begin{array}{l}\text { Impurity content of }<1 \% \\
\text { in plasi a chamber } \\
\text { after fueling }\end{array}$ \\
\hline Pumpdown time between burns ( $s$ ) & 30 & $\begin{array}{l}\text { Reasonable dwell time } \\
\text { between burns }\end{array}$ \\
\hline Conducting ratio, $\gamma(\%)$ & 20 & $\begin{array}{l}\text { Ensure adequate impurity } \\
\text { removal during the } \\
\text { burn }\end{array}$ \\
\hline
\end{tabular}




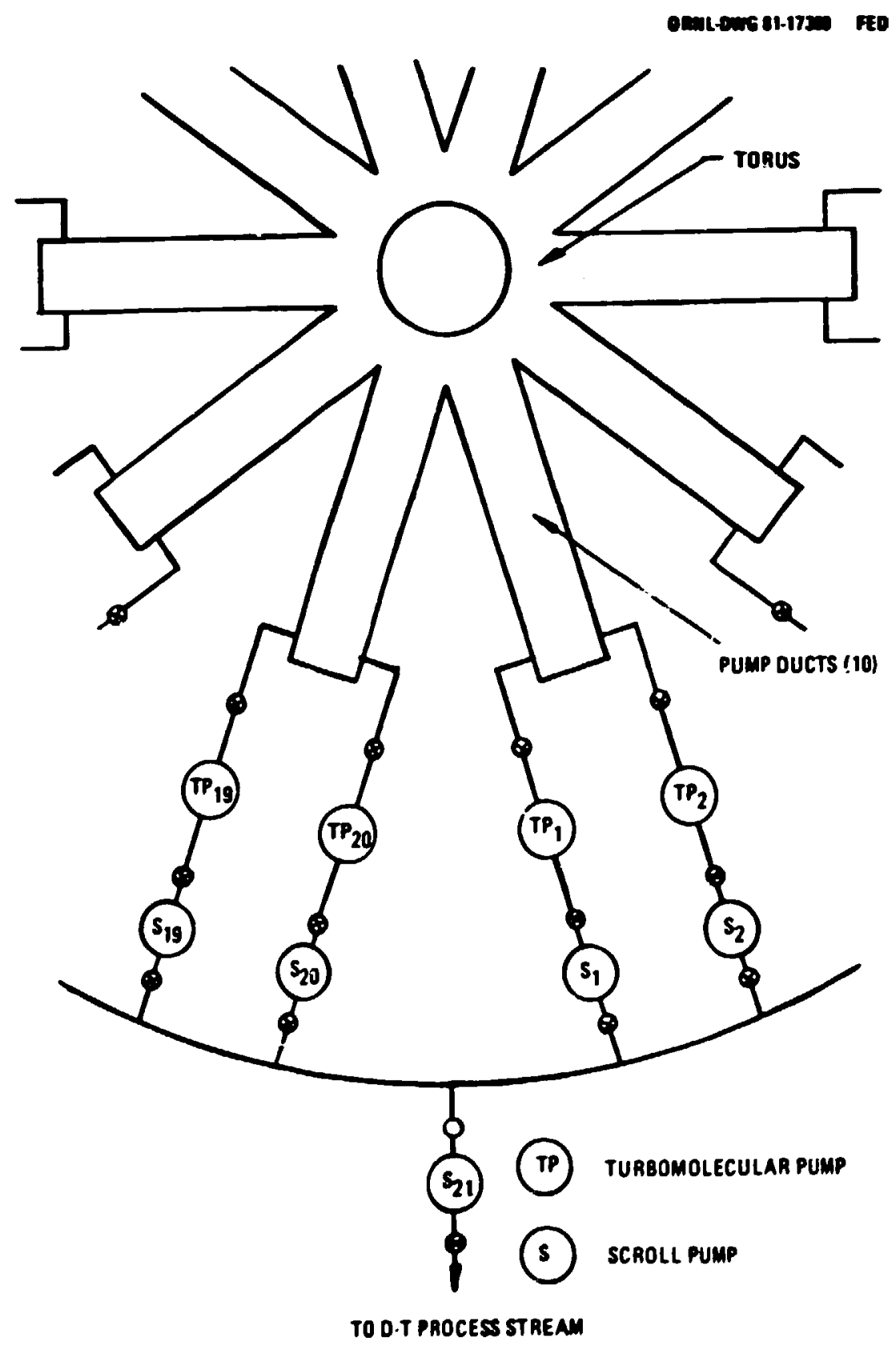

Fig. 11-1. Vacuum pumping system. 
apfroximately atmospheric pressure. The major design parameters of the vacuum pumps are summarized in Table 11.4 .

The high-vacuum turbomolecular pumps are required to have a speed of $5 \mathrm{~m}^{3} \cdot \mathrm{s}^{-1}$ for helium and hydrogen isotopes. They will have to be designed for tritium service; all seals will have to be metallic; and provisions will have to be made for remote oil change, maintenance, repair, and replacement. A further consideration is the possibility of developing a $5-\mathrm{m}^{3} \cdot \mathrm{s}^{-1}$ turbomolecular pump with magnetic or gas bearings to avoid the hazards associated with tritium-contaminated oil in conventional oil-bearing pumps.

Scroll pumns back the turbomolecular pumps and are used for rough pumping of the plasma chamber. These are sealed pumps that have no bearing lubricant in contact with the tritium stream. Two stages of scroll pumps are required to reach the pressure of $10^{-4}$ to $10^{-3}$ torr needed for effective pumping by the turbomolecular pumps to pressures below $10^{-7}$ torr. A first-stage scroll pump backs each turbomolecular pump, exhausting at a pressure of aboit 25 torr. The exhaust from all eight first-stage pumps is combined and ducted to a single, small, second-stage scroll pump that exhausts at about atmospheric pressure.

The effective pump speed of the total vacuum pumping system as currently designed is $18.5 \mathrm{~m}^{3} \cdot \mathrm{s}^{-1}$. The major limitations of the total system are the conductance of the $0.5-\mathrm{m}-\mathrm{diam}$ duct and the pump speed. A duct diameter greater than $0.5 \mathrm{~m}$ cannot be used for the reference TFCX major radjus, duct routing scheme, and TF coil configuration. The duct size can increase significantly if the major radius is increased. If the total pump speed is increased from $40 \mathrm{~m}^{3} \cdot \mathrm{s}^{-1}\left[8-5 \mathrm{~m}^{3} \cdot \mathrm{s}^{-1}\right.$ (e.g., $32-5 \mathrm{~m}^{3} \cdot \mathrm{s}^{-1}$ pumps or $8-20 \mathrm{~m}^{3} \cdot \mathrm{s}^{-1}$ pumps), the effective pump speed of the total system is increased to $33 \mathrm{~m}^{3} \cdot \mathrm{s}^{-1}$. If, in addition to the increased pump speed, a configuration that allowed $1.0-\mathrm{m}$-diam vacuum ducts was developed, the effective pump speed would be $60 \mathrm{~m}^{3} \cdot \mathrm{s}^{-1}$. The actual design parameters to be used for TFCX remain to be determined, but the vacuum pumping requirements may not be easily satisfied.

Evaluation of the plasma exhaust pumping indicates that the conductance ratio for the reference design is $5 \%$, which falls shor $i$ of the $20 \%$ guidelinc. By simply replacing the $5 \mathrm{~m}^{3} \cdot \mathrm{s}^{-1}$ pumps with $20 \mathrm{~m}^{3} \cdot \mathrm{s}^{-1}$ 
Table 11.4. Vacuum pump parameters

\begin{tabular}{ll}
\hline High-vacuum pumps & \\
Pump type & Turbomolecular \\
Pump speed for $\mathrm{H}_{2}$ and $\mathrm{H}_{\mathrm{e}}$ & $5 \mathrm{~m}^{3} \cdot \mathrm{s}^{-1}$ \\
Number of pump ducts & 16 \\
Number of pumps per duct & 1 pump per 2 ducts \\
Backing/rouging pumps & \\
Pump type & Scroll \\
Number of stages & 2 \\
Number cf first-stage pumps & 8 \\
Number of second-stage pumps & 1 \\
Speed of first-stage pump & $3 j \ell \cdot 5^{-1}$ \\
Inlet pressure & $10^{-4}$ to $10^{-3}$ torr \\
Speed of second-stage pump & $\sim 2 \ell \cdot 5^{-1}$ \\
Inlet pressure & 225 torrr \\
Discharge pressure & $\sim 1$ atm
\end{tabular}<smiles>CCCCCC</smiles> 
pumps, the conductance ratio is increased to $9 \%$. Although the diameter of the vacuum pumping ducts cannot be increased beyond $0.5 \mathrm{~m}$ in some spact-limited regions (e.g., between TF coils), significant inprovements may be possible by increasing the duct size in other regions. A major vacuum system configuration change may be possible in the region inmediately outboard of 4 of the 16 removable sector modules, and this would also improve the conductance ratio. Future effort is needed to determine which, if any, design changes arc required to establish adequate impurity removal efficienc;.

\subsection{TRITIUM SYSTEMS}

The critium systems serve two primary functions: (1) to provide new and/or recycled tritium and deuterium fuel for plasma operations and (2) to lim,t worker radiation exposure and to minimize tritium losses to the environment.

The TFCX class of fusion device will have a nominal $2 \times 10^{5} \mathrm{~s}$ of tritium operation over a lifetime of five to ten years. The power level is expected to range from 250 to $400 \mathrm{MW}$ with an assumed fractional burn of $2 \%$. This device uses a pellet fueler and turbomolecular pumps. Tritium neutral beams are not used. Their use would increase the tritium needed by a factor of 3 .

To assess the tritium supply, the tritium systems needed, and the environmental impact of this class of device, two burn times (20-s pulses and 300-s pulses) and different processing modes (on-site and off-site, continuous and batch) were considered.

A number of trade studies were performed to determine the effect of different factors in designing tritium systems for a TFCX class of device; (1) the effects of plasma chal scteristics on the amount of tritium needed; (2) an assessment of the hazars associated with transporting tritium; (3) tritium needs and the location within the plant for different processing scenarios; and (4) a summary of the advantages and disadvantages of different processing scenarios.

$\wedge$ reference case was developed that fulfills the requirements desired for plasma physics experiments. A complete fuel cycle is 
provided. This includes not only the components needed to process and store the fuel (fuel cleanup unit, isotope separatior unit, storage beds, receiving glovebox) but also the units required for secondary containment (glovebox detritiation system), waste procesing (tritium waste treatment - gases; tritiated water recovery unit - water and tritiated waste processing - solids), tritium analysis (required to detect problems and their solutions in the fuel cycle and to maintain accountability records), tritium monitoring (safety considerations), and process control with associated data acquisition system. Figure 11.2 shows the flow paths between the tritium systems.

The units are located in a building separate from the reactor that is leaktight and earthquake-proof. Earthquake valves are located on lines and between units to minimize any potential releases. An atmospheric processing system is in the tritium processing building to handle all tritium releases in the area. The floor plan has been arranged so as to have access to all units during maintenance and to minimize the length of connecting lines.

\subsection{REMOTE MAINTENANCE EQUIPMENT}

Remote maintenance procedures are required whenever it is necessary to remove or gain access to components comprising or located inside the. reactor radiation shield system. This includes removal of shield sectors and ancillary equipment shielding and access to conponents penetrating the shield envelope. In addition, the capability to maintain equipment outside the shield envelope by remote means has to be provided in order to accomplish at least some of the more straightforward operations during radiation cooldown and, if necessary, to carry out whatever operations are required while part of the shielding system is removed.

Two classes of remot = maintenance equipment are required: general purpose and special purposc. Special purpose equipment is required for handling and transporting components, for inspecting areas $f f$ concern, for detecting faults, for cutting and welding nonstandard joiats, and for making in situ repairs. General purpose devi:es such as manipulators 


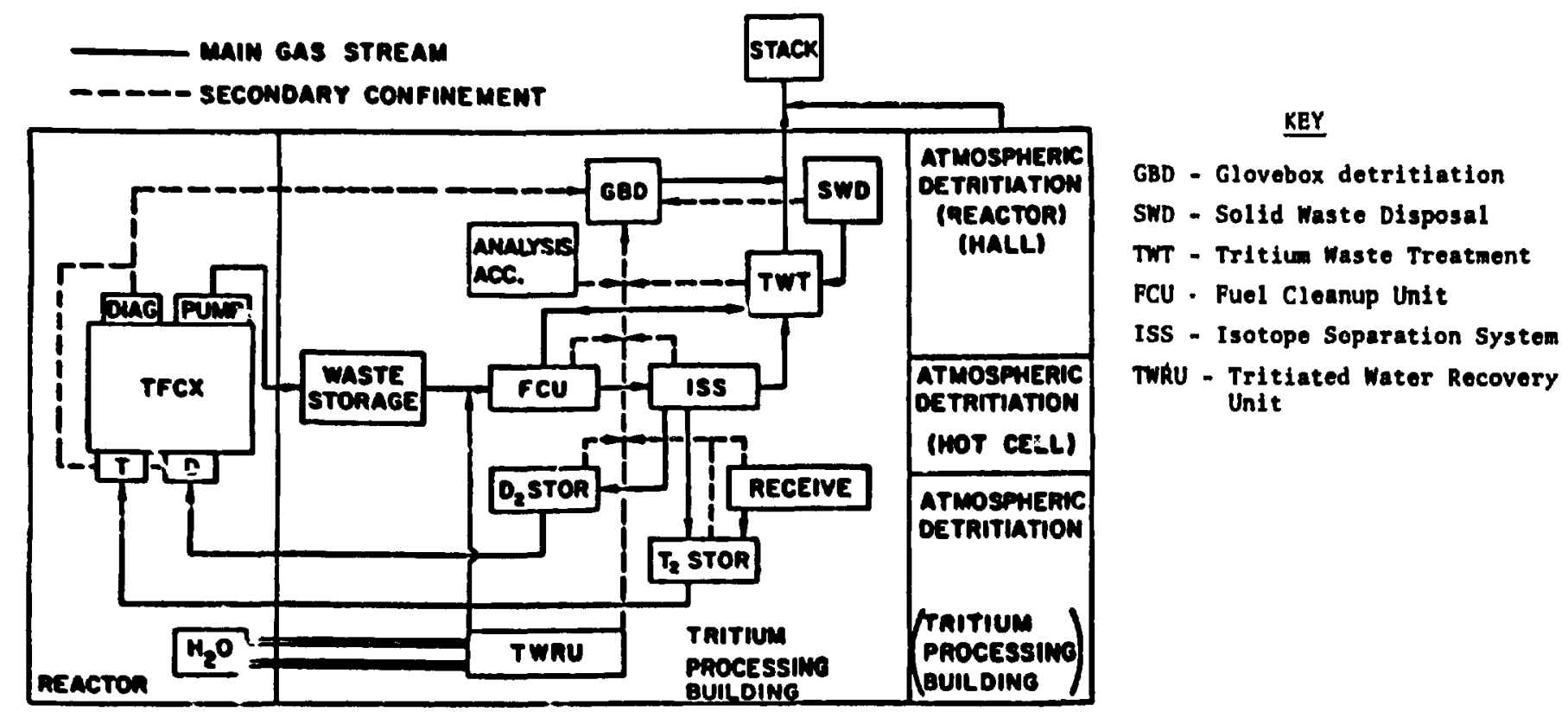

Fig. 11.2. Tritium flow paths for TFCX tritium processing units. 
and cranes, are required for operations involving tools and for handling components once they have been removed from the reactor. In addition, the general purpose equipment must have sufficient flexibility to perform unplanned maintenance operations. Although some limited design has been carried out on specific items of remote maintenance equipment, substantial additional work is required to better define the characteristics of the remote maintenance equipment and to establish a reliabie cost estimate. The specific requirements for TFCX have been assessed compared to the requirements established for the FED and the remote maintenance equipment needs have been defined particular to satisfying the mission requirements of the TFCX device. 
At present, facilities data are displayed locally and archived on tapes that may be recalled at the iocal control area. With the advent of a more unified fusion development program, the data can be made available to facilities at remote locations. The operational data can be provided to several institutions or laboratories as they are generated.

The control of the facility and the operation of the device will utilize the state-of-the-art digital control system. The TFCX instrumentation and control system will be based on the inclusion of dedicated local control and protection functions in each subsystem and between subsystens, where required. Integrated facility monitoring and operator interface functions will be provided by a central control facility, which will interface with and overlay subsystem controls. Diagnostic data acquisition will be accomplished by dedicated, modularized, highspeed data handling systems with interactive capability. Non-real-time - bulk data processing will be provided by a separate suitable facility. Plasma control is defined as feedback control on the plasma parameters such as plasma density, temperature, position, shape, fuel mixture, and volume. The plasma position control has been addressed on present machines. However, it will be different on TFCX because the structures will have nonuniform induced currents. Thus, the control field peletration will be very much nonlinear from a spatial consideration, and the induced fields or currents will be much larger in magnitude. The development of controls for regulating such items as plasma shape and plasma temperature will require a development program. The methods of measuring the shape and current densjties will require future component development. The method of controlling the plasma temperature and power output for a 300-s burn has not been selected.

The stabilization of the elongated plasma before the stage of ignition is reached may require development. The elongated plasma is much more conditionally unstable in the vertical direction than fn: the case of the nonelongated plasma. Several options are available. 


\section{FACIIITIES}

The plant facilities support the operation and maintenance of TFCX. These facilities house and provide a controlled environment for the TFCX equipment. A suggested site arrangement is given in Fig. 12.1.

The emphasis of the facilities effort for TFCX has focus.d on devising the necessary facilities to support a TFCX design at a new site. In addition, most of the effort has focused on the reactor building and hot cell complex. The remaining facilities have been adapted from the earlier FED studies with appropriate modifications to reflect the change in mission.

A primary feature of the TFCX facilities layout is that the reactor hali (RH), the decontamination and hot cell facility (HCF), the tritium processing area $(T)$, and the radioactive waste processing area (RW) all have been included on a common foundation mat. Figure 12.2 is a plan view of the TFCX nuclear island at two elevations (ground level and at $11 \mathrm{~m})$. This feature provides a number of advantages, including shared comnon walls, simplified and improved seismic response, enhanced radiological safety by providing a single point of access to all potential radiation hazards, and concentration of the air-detritiation system into a single structure. The result is a much improved concept that is simpler and less costly. 
TFCX FUSION ISLAND A
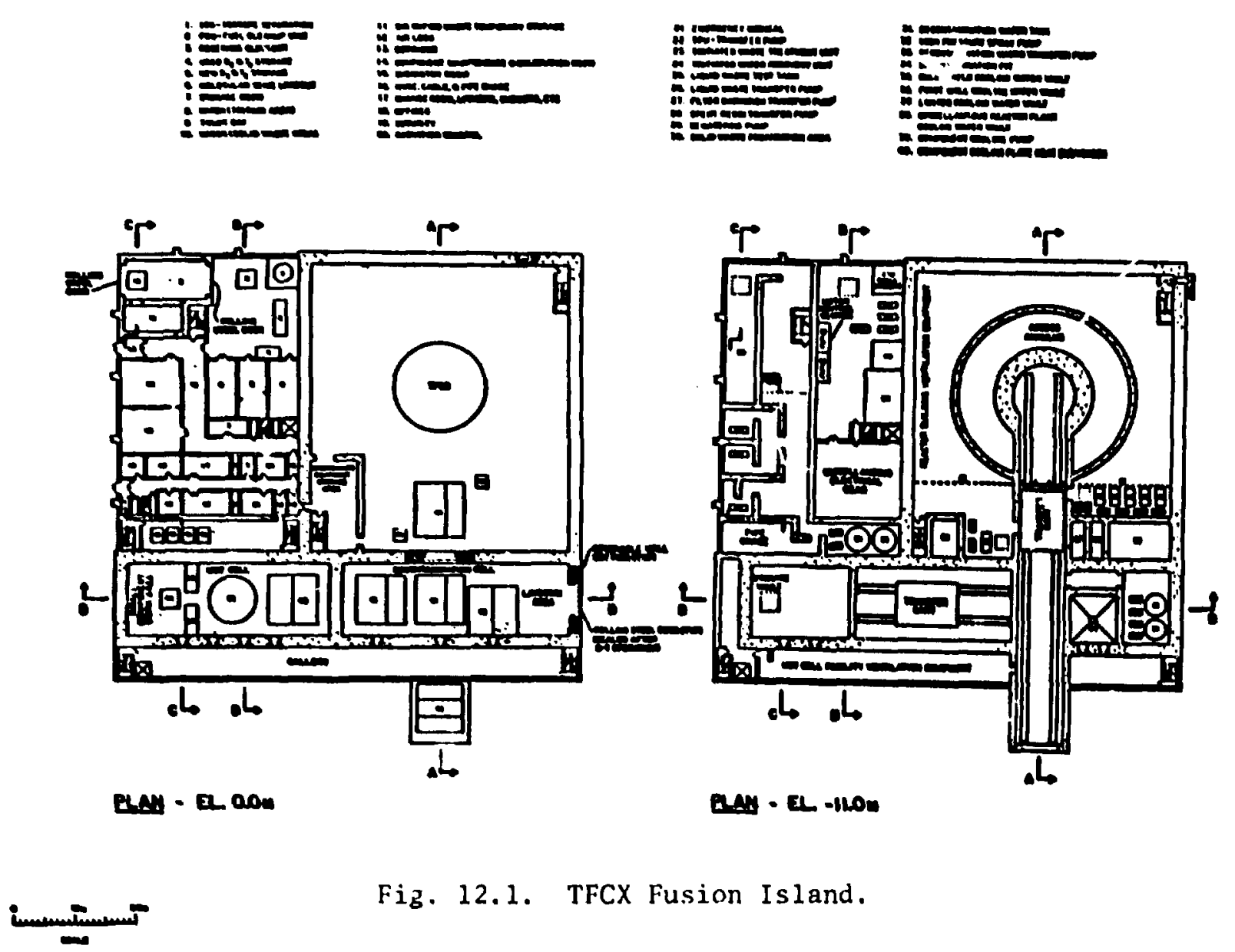

Fig. 12.1. TFCX Fusion Island. 


\section{TFCX PLOT PLAN}

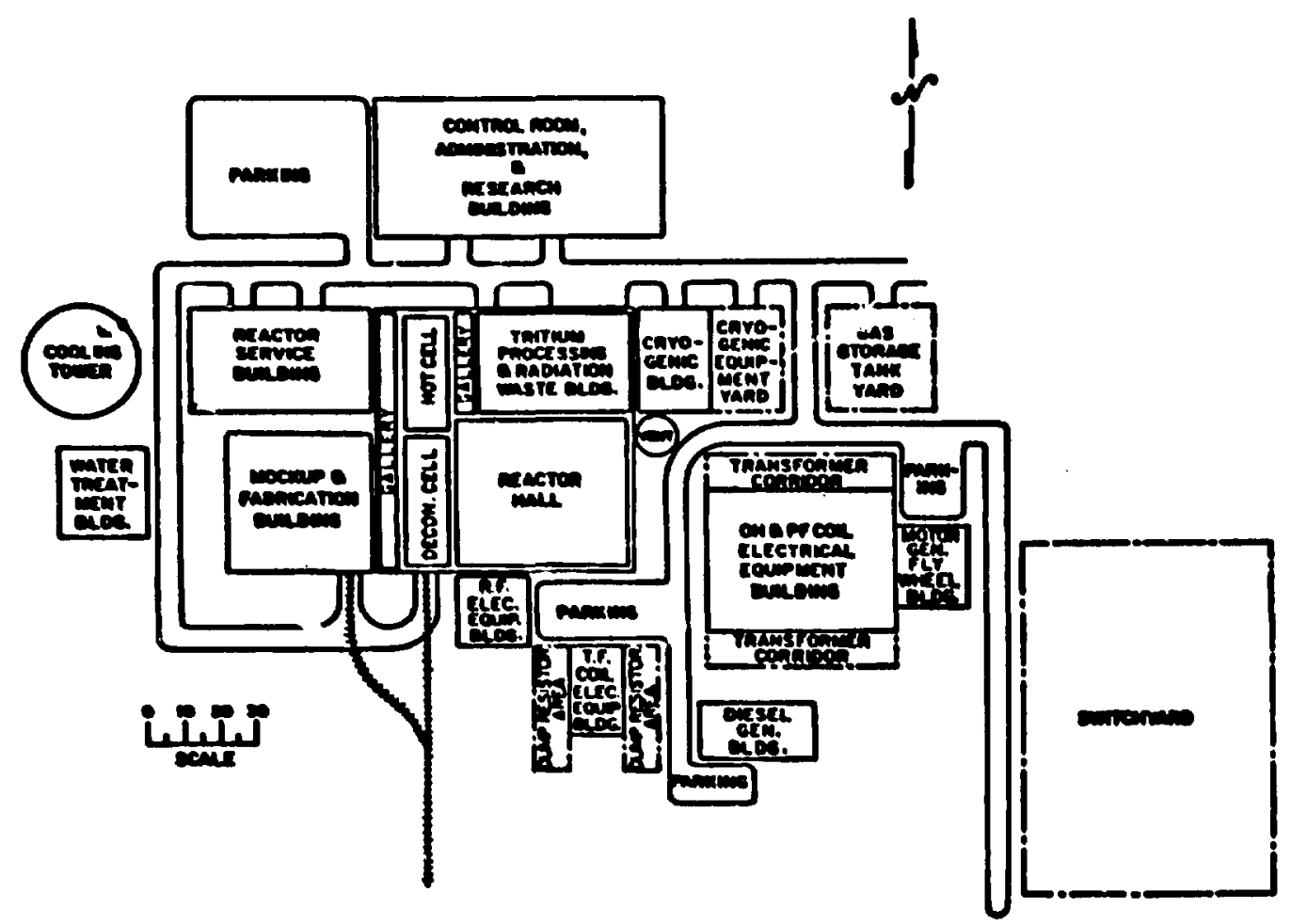

Fig, 12.?. TFCX plot plan. 


\section{CONCLUDING REMARKS}

The design and trade study information presented in this ducument represents a strong basis. for understanding. the superconducting and superconducting-copper hybrid TF coil options as candidates for TFCX. These designs are based on many years of development in reactor studies performed in the United States and around the world. They make use of the latest thinking regarding component design of the major systems and components, in particular, the design of the magnet systems, the torus systems, the impurity control systems, the plasma heating systems, and the facilities. The physics assumptions regarding confinement scaling are subject to improvement as new experimental information becomes available. However, the latest physics information has been incorporated into the TFCX design.

This interim documert summarizes design issues, anaiysis, trade studies, and an ongoing effort to present a total, integrated, selfconsistent design for the superconducting TF coil options for TFCX. Subsequent documentation will update the design evolution. 
ORNL $j$ FEDC-84/3

Dist. Category UC-20 c,d

INTERNAL DISTRIBUTION

1. S. E Attenberger

2. R. J. Barrett

3. D. D. Bates

4. L. A. Berry

5. D. T. Blackfield

6. S. K. Borowski

7. T. G. Brown

8. G. T. Bussell

9. B. A. Carreras

10. R. J. Colchin

11. A. Dabiri

12. R. A. Dory

13. J. L. Dunlap

14. A. C. England

15-19. C. A. Flanagan

20. J. D. Galambos

21. G. E. Gorker

22. J. R. Haines

23. W. R. Hanilton

24. H. H. Haselton

25. P. N. Haubenreich

26. D. L. Hillis

27. L. M. Hively

28. J. A. Holmes

29. R. J. Hooper

30. W. A. Houlberg

31. T. C. Jernigan

32. S. S. Kalsi

33. D. C. Keeton

34. P. King

35. J. Kirchner

36. V. D. Lee

37. C. M. Loring

38. D. C. Lousteau
39. M. S. Lubell

40. J. F. Lyon

41. T. L. Mann

42. D. H. Metzler

43. C. E. Nelson

44. W. D. Nelson

45. Y-K. M. Peng

46. R. L. Reid

47. B. W. Reimer

48. J. A. Rome

49. K. E. Rothe

50. M. J. Saltmarsh

51. J. L. Scott

52. T. E. Shannon

53. J. Sheffield

54. P. T. Spampinato

55. D. Spong

56. V. C. Srivastava

57. D. J. Strickler

58. P. B. Thompson

59. S. L. Thomson

60. N. A. Uckan

61. T. Uckan

62. T. L. White

63. F. W. Wiffen

64. K. F. Wu

65-66. Laboratory Records Depariment

67. Laboratory Records, ORNL-RC

68. Document Reference Section

69. Central Research Library

70. Fusion Energy Division Library

71. Fusion Energy Division Publications Office

72. ORNL Patent Office 


\section{EXTERNAL DISTRIBUTION}

73. M. A. Abdou, Associate Director, FPP/207, Argonne National Lahuratory, 9700 South Cass Avenue, Argonne, IL 60439

74. C. A. Anderson, Westinghouse Electric Corporatior:, Advanced Energy Energy Systems Division, P.O. Box 158, Madison, PA 15663

75. I. L. Anderson, CMB-3, Mail Stop 348, Los Alamos National Laboratory, P.O. Box 1633, Los Alamos, NM 87545

76. C. C. Bz.ker, FPP/208, Argonne National Laboratory, 9700 South Cass Avenue, Argonne, IL 60439

77. D. S. Beard, Office of Fusion Energy, Office of Energy Research, Mail Stop G-256, U.S. Department of Energy, Washington, DC 20545

78. K. L. Black, Department E452, McDonnell Douglas Astronautics Company, P.O. Box 516, St. Louis, MO 63166

79. R. Botwin, C47-05, Grumman Aerespace Corporation, P.O. Box 31, Bethpage, NY 11714

80. W. B. Briggs, McDonnell Douglas Astronautics Company, P.O. Box 516, St. Louis, MO 63166

81. J. N. Brooks, FPP/207, Argonne National Laboratory, 9700 South Cass Avenue, Argonne, IL 6 G439

82. S. C. Burnett, GA Technologies, Inc. P.O. Box 81608, San Diego, CA 92138

83. J. D. Callen, Department of Nuclear Engineering, University of Wisconsin, Madison, WI 53706

84. D. R. Cohn, MIT Plasma Fusion Center, 167 Albany Street, Cambridge, MA 02139

85. J. W. Coursen, C36-05, Grur:aman Aerospace Corporation, P.O. Box 31, Bethpage, NY 11714

86. R. W. Conn, School of Chemical, Nuclear, and Thermal Engineering, Boelter Hall, University of California, Los Angeles, CA 90024

87. J. G. Crocker, EG\&G Idaho, P.O. Box 1625, Idaho Falls, ID 83401

88 G. R. Dalton, Department of Nuclear Engineering Science, Nuclear Srience Center, University of Florida, Gainesville, FL 32611

89. R. C. Davidson, Massachusetts Insiilute of Tecinology, 77 Massachusetts Avenue, Cambridge, MA 02139

90. N. A. Davies, Office of Fusical Energy, Office of Energy Research, Mail Stop G-2.56, U.S. Department of Energy, Washington, DC 20545

91. S. O. Dean, Director, Fusion Energy Development, Science Applications, Inc., 2 Professional Drive, Suite 249, Gaithersburg, MD 20760

92. J. F. Decker, Office of Fusion Energy, Maii Stop G-256, U.S. Department of Energy, Washington, DC, 20545

93. D. DeFreece, EA51, Building 81/1/B7, McDonnell Douglas Astronautics Company, P.O. Box 516, St. Louis, MO 63166 
94. J. N. Doggett, L-441, Lawrence Livermore National Laboratory, P.O. Box 808, Livermore, CA 94550

95. H. Dreicer, Division Leader, CRT, Los Alamos National Laboratory, P.O. Box 1663, Los Alamos, NM 87545

96. D. Ehst, Argonne National Laboratory, 9700 South Cass Avenue, A gonne, IL 60439

97. G. A. Elissev, I. V. Kurchatov Institute of Atomic Energy, P.O. Box 3402, 123182 Moscow, U.S.S.R.

98. F. Farfaletti-Casali, Engineering Division, Joint Research Center, Ispra Establishment, 21020 Ispra (Varese), Italy

99. P. A. Finn, Fusion Program, Argonne National Laboratory, 9700 South Cass Avenue, Argonne, IL 60439

100. H. K. Forsen, Bechtel Group, Inc., Research \& Engineering, P.O. Box 3965, San Francisco, CA 94119

101. J. S. Foster, Jr., Building R4-2004, TRW Defense and Space Systems, 1 Space Park, Redondo Beach, CA 90278

102. T. K. Fowler, Associate Director for Magnetic Fusion Energy, L-436, Lawrence Livermore National Laboratory, P.O. Box 808, Livermore, CA 94550

103. J. W. French, EBASCO Services, Inc., Forrestal Campus, CN-59, Frinceton University, Princeton, NJ 08544

104. H. P. Furth, Director, Princeton Plasma Physics Laboratory, P.O. Box 451, Princeton, NJ 08544

105. J. G. Gavin, Jr., President, A01-11, Grumman Aerospace Corporation, P.O. Box 31, Bethpage, NY 11714

106. G. Gibson, Westinghouse Electric Corporation, Fusion Power S; stems Department, P.O. Box 10864, Pittsburgh, PA 15236

107. J. R. Gilleland, Manager, Fusion Project, GA Technologies, Inc., P.O. Box 81608 , San Diego, CA 92138

108. V. A. Glukhikh, Scientific-Research Institute of Electro-Physical Apparatus, 188631 Leningrad, U.S.S.R.

109. M. Y. Sohar, Argonne National Laboratory, 9700 South Cass Avenue, Argonne, IL 60439

110. R. W. Gould, Department of Applied Physics, California Institute of Technology, Pasadena, CA 91109

111. M. W. Griffin, Department E236. McDonnell Douglas Astronautics Company, P.O. Box 516, St. Louis, MO 63166

112. C. R. Head, Office of Fusion Energy, Mail Stop G-256, U.S. Department of Energy, Washington, DC 20545

i13. C. D. Henning, Lawrence Livermore National Lahoratury, P.O. Box 808, Livermore, CA 94550

114. J. J. Holmes, Westinghouse-Hanford Engineering Development Laboratory, P.O. Box 1970, Richland, WA 99352 
115 D. Hwang, Princeton Plasma Physics Laboratory, P.O. Box 451, Princeton, NJ 08544

116. J. B. Joyce, Princeton Plasma Physies Laboratory, P.O. Box 451, Princeton, NJ 08544

117. R. A. Krakowsti, CTR-12, Mail Stop 641, Los Alamos National Laboratory, P.O. Box 1663, Los Alamos, NM 87545

118. G. L. Kulcinski, University of Wisconsin, Department of Nuclear Engineering, Engineering Research Building, Room 439, 1500 Johnson Drive, Madison, WI 53706

119. D. L. Kummer, McDonnell Douglas Astronautics Company, P.O. Box 516, St. Louis, 1.1063166

120. D. G. McAles, Exxon Nuclear Company, Inc., 777 106th Avenue, NE, Bellevue, WA 98009

121. W. Marton, Office of Fusion Eacrgy, Office of Energy Research, Mail Stop G-256, U.S. Department of Energy, Washington, DC 20545

122. L. G. Masson, EG\&G Idaho, Idaho National Engineering Laboratory, P.O. Box 1625, Idaho Falls, ID 83401

123. D. M. Meade, Princeton Plasma Physics Laboratory, P.O. Box 451, Princeton, NJ 08544

124. A. T. Mense, Building 107, Post B2, McDonnell Douglas Astronautics Company, P.O. Box 516, St. Louis, MO 63166

125. R. W. Moir, Lawrence Livermore Laboratory, P.O. Box 808, Livermore, CA 94550

126. D. B. Montgomery, MIT Plasma Fusion Center, 167 Albany Street, Cambridge, MA $021 ? 9$

127. A. E. Munier, Grumman Acrospace Company, P.O. Box 31, Bethpage, NY 11714

128. R. E. Nygren, FPP/207, Argonne Nationai Laboratory, 9700 South Cass Ave.ilue, Argonne, $1 \mathrm{~L} 60439$

129. T. Ohkawa, GA Technologies, Inc., P.O. Box 81608, San Diego, CA 92138

130. J. A. O'Toole, Plasma Physics Laboratory, James Forrestal Campus, P.O. Box 451, Building I-P, Room 8A, Princeton, NJ 08544

131. R. R. Parker, Francis Bitter National Magnet Laboratory, 170 Albany Street, Cambridge, MA 02139

132. B. Pease, Culham Laboratory, Abingdon, Oxfordshire OXI4 3DB, United Kingdom

133. M. Pelovitz, Princeton Plasma Physics Laboratory, P.O. Box 451, Princeton, NJ 08544

134. F. W. Perkins, Princeton Plasma Physics Laboratory, P.O. Box 451, Princeton, NJ 08544

135. M. Porkolab, Massachusetts Institute of Technology, 77 Massachusetts Avenue, Cambridge, MA 02139

136. D. E. Post, Princeton Plasma Physics Laboratory, P.O. Box 451, Princeton, NJ 08544

137. L. K. Price, Department of Energy, Oak Ridge Operations, P.O. Box E, Oak Ridge, TN 37830 
138. R. E. Price, Office of Fusion Energy, Office of Energy Research, Mail Stop G-256, U.S. Department of Energy, Washington, DC 20545

139. F. A. Puhn, GA Technologies, Inc., P.O. Box 81508, San Diego, CA 92138

140. J. Purcell, GA Technologies, Inc., P.O. Box 81608, San Diego, CA 92138

141. R. V. Pyle, University of California, Lawrence Berkeley Laboratory, Berkeley, CA 94720

142. J. M. Rawls, GA Technologies, Inc., P.O. Box 81608, San Diego, CA 92138

143. P. J. Reardon, Princeton Plasma Physics Laboratory, P.O. Box 451, Princeton, NJ 08544

144. M. Roberts, Office of Fusion Energy, Mail Stop G-256, U.S. Department of Energy, Washington, DC 20545

145. J. D. Rogers, Los Alamos National Laboratory, P.O. Box 1663, Los Alamos, NM 87545

146. M. L. Rogers, Monsanto Research Corporation, Mound Laboratory Facility, P.O. Box 32, Miamisburg, OH 45342

147. M. N. Rosenbluth, RI.M 11.2!8, Institute for Fusion Studies, University of Texas, Austin, TX 78712

148. P. H. Rutherford, Princeton Plasma Physics Laboratory, P.O. Box 451, Princeton, NJ 08544

149. D. D. Ryutov, Institute of Nuclear Physics, Sikerian Branch of the Academy of Sciences of the U.S.S.R., Sovetskaya St. 5, 630090 Novosibirsk, U.S.S.R.

i50. J. A. Schmidt, Princeton Plasma Physics Laboratory, P.O. Box 451, Princeton, NJ 08544

151. J. Schultz, MIT Plasma Fusion Center, 167 Albany Street, Cambridge, MA 02139

152. F. R. Scott, Electric Power Research Institute, P.O. Box 10412, Palo Alto, CA 94304

153. G. Sheffield, Princeton Plasma Physics Laboratory, P.O. Box 451, Princeton, NJ 08544

154. D. Smith, Materials Science Division, Argonne National Laboratory, 9700 South Cass Avenue, Argonne, IL 6C439

155. L. Southworth, GA Technologies, Inc., P.O. Box 81608, San Diego, CA 92138

156. I. Spighel, Lebedev Physical Institute, Leninsky Prospect 53, 117924 Moscow, U.S.S.R.

157. W. M. Stacey, Jr., Georgia Institute of Technology, School of Nuclear Engineering, Atlanta, GA 30332

158. E. Stern, Grumman Aerospace Corporation, CN-59, Forrestal Campus, Princeton, NJ 08544

159. P. M. Stone, Office of Fusion Energy, Office of Energy Research, Mail Stop G-256, U.S. Department of Energy, Washington, DC 20545

160. I. N. Sviatoslavsky, Room 33, Engineering Research Building, 1500 Johnson Drive, University of Wisconsin, Madison, WI 53706 
161. R. E. Tatro, Manager, Energy Systems, M.Z. 16-1070, General Dynamics-Convair Division, P.O. Box 80847, San Diego, CA 92138

162. F. Thomas, B-20-5, Grumman Aerospace Curporation, Bethpage, NY 11714

163. K. I. Thomassen, Lawrence Livermore National Laboratory, P.O. Box 808, Livermore, CA 94550

164. R. J. Thome, Francis Bitter National Magnet Laboratory, 170 Albany Street, Cambridge, MA 02139

165. V. T. Tolok, Kharkov Physical-Technical Institute, Academical St. 1, 310108 Kharkov, U.S.S.R.

166. C. Trachsel, McDonnell Dnuglas Astronautics Company, P.O. Box 516, St. Louis, MO 63166

167. A. W. Trivelpiece, Office of Fusion Energy, Office of Energy Research, Mail Stop G-256, U.S. Department of Energy, Washington, DC 20545

168. L. R. Turner, Fusion Power Program, Argonne National Laboratory. 9700 South Cass Avenue, Argonne, IL 60439

169. E. H. Valeo, Princeton Plasma Physics Laboratory, P.O. Box 451, Princeton, NJ 08544

170. R. Varma, Physical Research Laboratory, Navangpura, Ahmedabad, India

171. K. E. Wakefield, Princeton Plasma Physics Laboratory, P.O. Box 451, Princeton, NJ 08544

172. J. C. Wesley, GA Technologies, Inc., P.O. Box 81608, San Diego, CA 92138

173. H. Willenberg, Mathematical Sciences Northwest, Inc., P.O. Box 1887, Bellevue, WA 98009

174. J. E. C. Williams, Francis Bitter National Magnet Laboratory, 170 Albany Street, Cambridge. MA 02139

175. H. H. Yoshikawa, W/A-62, Hanford Engineering Developmei:. Laboratory, P.O. Box 1970, Ricklland, WA 99352

176. K. M. Young, Princeton Plasma Physics Laboratory, P.O. Box 451, Princeton, NJ 08544

177. N. E. Young, EBASCO Services, Inc., Princeton Plasma Physics Laboratory, P.O. Box 451, Princeton, NJ 08544

178. Bibliothek, Max-Planck Institut fur Plasmaphysik, D-8046 Garching bei Munchen, Federal Republic of Germany

179. Bibliothek, Institut fur Plasmaphysik, KFA, Postfach 1913, D-5170 Julich, Federal Republic of Germany

180. Bibliotheque, Service du Confinement des Plasmas, CEA, B.P. No. 6, 92 Fontenay-aux-Roses (Seine), France

181. Documentation S.I.G.N., Department de la Physique du Flasma et de la Fusion Controlee, Association EURATOM-CEA, Centre d'Etudes Nucleaires, B.P. 85, Centre du Tri, 38041 Grenoble, Cedex, France

182. Library, Centre de Recherches en Physique des Plasmas, 21 Avenue des Bains, 1007 Lausanne, Switzerland 
183. Library, Culham Laboratory, UKAEA, Abingdon, Gxfordshire, OXI4 3DB, England

184. Library, FOM Institut voor Plasma-Fysica, Rijnhuizen, Jutphaas, Netherlands

185. Library, Institute of Physics, Academia Sinica, Beijing, Peoples Republic of China

186. Library, Institute for Plasma Physics, Nagoya University, Nagoya 464, Japan

187. Library, International Centre for Theoretical Physics, Trieste, Italy

188. Library, JET Joint Undertaking, Abingdon, Oxfordshire, OX14, DB, England

189. Library, Laboratoria Gas Ionizzati, Frascati, Italy

190. Plasma Research Laboratory, Australian National Laboratory, P.O. Box 4, Canberra. ACT 2000, Australia

191. Thermonuclear Library, Japan Atomic Energy Research Institute, Tokai, Naka, Ibaraki, Japan

192. Library, Pla na Physics Laboratory, Kyoto University, Gokasho Uji, Kyoto, Japan

193. Office of the Assistant Manager for Energy Research and Development, U.S. Department of Energy, Oak Ridge Operations, Oak Ridge, TN 37830

194-383. Given distribution as shown in TID-4500, Magnetic Fusion Energy (Distribution Category UC-20 c,d: Reactor Materials and Fusion Systems) 